\title{
EVALUACIÓN Y APORTACIONES A LAS \\ NUEVAS NORMATIVAS ANTE \\ PERTURBACIONES ELÉCTRICAS EN \\ INSTALACIONES DE ENERGÍAS RENOVABLES
}

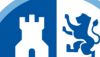

is

$+1$

Universidad

Politécnica

de Cartagena

\section{MEMORIA DE TESIS DOCTORAL}

Autora: Tania García Sánchez

Departamento de Ingeniería Eléctrica

Escuela Técnica Superior de Ingeniería Industrial

Universidad Politécnica de Cartagena

Cartagena, Febrero de 2015 


\title{
EVALUACIÓN Y APORTACIONES A LAS NUEVAS NORMATIVAS ANTE PERTURBACIONES ELÉCTRICAS EN INSTALACIONES DE ENERGÍAS RENOVABLES
}

\author{
Programa de Doctorado en Energías Renovables
}

Autora: Tania García Sánchez

Directores

Dr. Ángel Molina García

Dr. Emilio Gómez Lázaro

Departamento de Ingeniería Eléctrica

Escuela Técnica Superior de Ingeniería Industrial

Universidad Politécnica de Cartagena

Cartagena, Febrero de 2015 
A mis hijos 



\section{Agradecimientos}

Llegado este punto de la tesis donde tienes que mirar atrás para ver quién te ha ayudado y darle las gracias, me doy cuenta de lo afortunada que soy, y de la suerte que tengo con las personas que he tenido alrededor durante estos años.

No todo el mundo está contengo con sus directores, yo sin embargo estoy encantada. Cuando decidí embarcarme en este proyecto no era una estudiante recién salida de la carrera, sino una persona con 3 niños que no podía dedicar el $100 \%$ del tiempo a la tesis, tenía que desempolvar muchos conocimientos y adquirir otros más. Ellos han sabido dar con un tema que me ha apasionado, también han sabido tirar de mí sin agobiarme, respetando mi ritmo sin dejar que me duerma, y lo más importante, me han ayudado muchísimo. Me he sentido muy a gusto trabajando con ellos y si tuviera que empezar otra vez lo volvería a repetir sin dudarlo.

Tengo muy buenos compañeros de trabajo, para los cuales no tengo más que buenas palabras, que me han ayudado y apoyado siempre. Un detalle que me gustó mucho fue con Sergio, él estaba desbordado con su tesis porque la entregaba esa misma semana y yo estaba atascada con matlab, aparcó su tesis y con toda la paciencia del mundo me ayudó perdiendo un tiempo que en esos momentos es oro cuando era algo que podía haber hecho a la semana siguiente, a lo mejor es un gesto muy normal, pero para mí fue muy importante. Aunque no los nombre uno a uno, es verdad que puedo presumir de todos y cada uno de ellos.

Me cuesta mucho buscar las palabras para expresar mi gratitud a mi familia, no sé. Lo que han hecho ellos no lo hace todo el mundo. Las horas, días, años que me han ayudado con mis hijos, que los han cuidado, mimado, ayudado con sus deberes, exámenes,... La paciencia que han tenido con mis cambios de humor, el ayudarme con mis estancias...Soy muy consciente que lo que ellos han hecho por mí no lo hace todo el mundo.

Definitivamente soy la persona más afortunada del mundo. 



\section{Resumen}

La presente tesis doctoral analiza y evalúa la situación actual en cuanto a normativas de conexión a red se refiere, aportando una nueva estrategia de caracterización de huecos de tensión con el fin de facilitar su comparativa frente a los requerimientos actualmente en vigor. Por otro lado, y atendiendo a la falta de contribuciones para analizar un elevado número de eventos en términos de severidad y de similitud con las limitaciones actuales, técnicas estadísticas basadas en Análisis por Componentes Princpales y procesos clustering han sido propuestas y evaluadas. Estas técnicas permiten deducir patrones-tipo de huecos de tensión, ofreciendo una herramienta visual y novedosa con la que comparar trayectorias de tensión eficaz más probables y limitaciones impuestas por los operadores del sistema. Las metodologías aportadas en esta tesis suponen una herramienta genérica con la que caracterizar y evaluar huecos de tensión, tanto equilibrados como desequilibrados, independientemente de los requisitos marcados por cada normativa. Este aspecto dota de un valor añadido a la metodología aquí desarrollada, abriendo un campo de nuevas aplicaciones entre las que se incluye la comparativa de campañas de medidas para diferentes emplazamientos o sistemas potencia.

Con el fin de evaluar la idoneidad de la metodología aquí propuesta se han caracterizado y analizado un conjunto de más de trescientas perturbaciones medidas en parques eólicos e instalaciones fotovoltaicas conectadas al sistema eléctrico español. Estos eventos son el resultado de un conjunto de campañas de medida llevadas a cabo a lo largo de varios años y lideradas la mayoría de ellas por el Instituto de Energías Renovables de la Universidad de Castilla La Mancha (España). Los resultados, patrones de comportamiento y comparativa con los requerimientos actuales del sistema eléctrico español suponen el ejemplo práctico de aplicación de las principales contribuciones de esta tesis a un caso práctico de instalaciones de energías renovables. 



\section{Abstract}

The present thesis describes and analyzes current grid-code requirements for generating units connected to the grid and submitted to disturbances. Under this framework, an alternative way to characterize voltage dips is proposed, making easier the comparison between real events and norms, in terms of rms-voltage trajectories and maximum duration given by the Transmisssion System Operators. Additionally, and due to the lack of contributions focused on analyzing and characterizing a large amount of events, statistics techniques based on Principal Component Analysis and K-means clustering processes have been discussed and implemented as well. The application of these techniques allows us to estimate a reduced number of rms-voltage patterns, corresponding with the most expected rms-voltage trajectories from a group of disturbances. This is a significant contribution of this thesis, providing a novelty solution to compare real rms-voltage and requirements by means of a reduced number of averaged rms-voltage profiles. Moreover, this approach is a generic tool to be applied on symmetrical and unsymmetrical disturbances, being independent on the grid-code considered. It thus offers a relevant added value for future applications, such as a comparison between different field-measurement campaigns, localizations or power system requirements.

With the aim of evaluating the suitability of the proposed techniques, over three hundred disturbances collected in Spanish wind farms and PV installations have been characterized. In addition, the most likely rms-voltage trajectories from the application of Principal Analysis Component and Kmeans clustering processes have been estimated as well. These events were collected along different field-measurement campaigns mainly promoting by the Institute of Renewable Energy, Universidad de Castilla La Mancha (Spain). Consequently, the estimated rms-voltage trajectories, the corresponding rms-voltage patterns as well as the comparison with current Spanish requirements suposses a practical example of application for the main contributions of this thesis. Moreover, this is a real and practical case study focused on current renewable installations connected to the grid. 



\section{Índice}

Agradecimientos $\quad$ V

Resumen VII

\begin{tabular}{lll}
\hline Abstract IX & IX
\end{tabular}

1. Aims and Motivations. 1

1.1. Aims . . . . . . . . . . . . . . . 2

1.2. Justification of the Interest and Motivations. . . . . . . . . 3

\begin{tabular}{ll}
\hline 2. Normativa y Requerimientos de Conexión & 7
\end{tabular}

2.1. Introducción. . . . . . . . . . . . . . . . 8

2.2. El Sector Eólico y Fotovoltaico. . . . . . . . . . . . . . . 11

2.3. Requerimientos de Conexión . . . . . . . . . . . . . . . 21

2.4. Propuestas en Estudio de Comportamiento ante Perturbaciones. 30

2.4.1. Propuesta Española. . . . . . . . . . . . . . . . 30

2.4.2. Propuesta de ENTSO-E. . . . . . . . . . . . . . 33

3. Caracterización de Huecos de Tensión 35

3.1. Introducción. . . . . . . . . . . . . . 36

3.2. Caracterización y Rrepresentación de Huecos de Tensión . . . 40 3.2.1. Caracterización mediante RMS. . . . . . . . . . . 40

3.2.2. Caracterización mediante componentes dq. . . . . . . 45

3.3. Propuesta de Inclusión de Severidad de Hueco. . . . . . . . . 49

3.3.1. Plantemiento General. . . . . . . . . . . . . . . . 49

3.3.2. Propuesta de severidad en estudios basados en RMS. . 52

3.4. Nuevas Propuestas de Caracterización . . . . . . . . . . 53

4. Identificación de Patrones de Huecos de Tensión 59

4.1. Planteamiento de la Problemática. . . . . . . . . . . . 60

\begin{tabular}{ll}
\hline 4.1.1. Métodos de Caracterización para Conjuntos \\
de Huecos de Tensión. Estado del Arte. . . . . . . . 60
\end{tabular} 
4.1.2. Evaluación de Métodos de Caracterización mediante Campañas de Medidas. . . . . . . . . . . . . 61

4.1.3. Métodos de Caracterización y Comparativa ante Requerimientos de Operación. . . . 66

4.2. Caracterización de Campañas de Medida . . . . . . . . . . . 68

$4.3 . \quad$ Metodología Propuesta . . . . . . . . . . . . . . . . . . 72

4.3.1. Análisis por Componentes Principales.

Descripción General. . . . . . . . . . . . . . . . . . . . 72

4.3.2. Agrupación basada en K-means.

Identificación de Patrones. . . . . . . . . . . . . . . . 75

4.4. Resultados . . . . . . . . . . . . . . . . . . . . . . . . . 78

4.4.1. Aplicación ACP y K-means a campañas de medida en parque eólico. . . . . . . . . . . . . . . . . . . . . . 78

4.4.2. Aplicación ACP y K-means a campañas de medida en instalaciones FV. . . . . . . . . . . . . . 83

5. Contributions and Future Work $\quad 89$

5.1. Contributions . . . . . . . . . . . . . 90

5.2. Future Work . . . . . . . . . . . . . . . . 92

\begin{tabular}{ll}
\hline Bibliografía & 95
\end{tabular} 


\section{Índice de figuras}

2.1. Comparativa de producción en función del tipo de combustible en Europa. . . . . . . . . . . . . . . . . . . . . . . . . . 10

2.2. Potencia generada neta durante 2000-2013. . . . . . . . . . . 12

2.3. Nueva potencia instalada y potencia desconectada en 2013. $\quad 13$

2.4. Potencia eólica instalada en la UE durante 2000-2013. . . . . 14

2.5. Potencia FV instalada en Europa durante 2000-2013. . . . . . 15

2.6. Potencia FV instalada en Alemania durante 2000-2013. . . . . 16

2.7. Potencia FV instalada en España durante 2000-2013. . . . . . 17

2.8. Potencia FV instalada en Italia durante 2008-2013. . . . . . . 17

2.9. Potencia eléctrica eólica proyectada para 2020. . . . . . . . . 19

2.10. Potencia eléctrica solar proyectada para 2020. . . . . . . . . . 20

2.11. Requerimientos de conexionado para parques eólicos: Comparativa de las curvas del valor límite de tensión eficaz en el punto de conexión a la red [1].]. . . . . . . . . . . . . . . . . 23

2.12. Requerimientos de conexión alemán: Curva límite de tensión para las plantas de generación tipo-1 en el punto de conexión a la red. .................. 25

2.13. Requerimientos de conexión alemán: Curva límite de tensión para las plantas de generación tipo-2 en el punto de conexión a la red. .................. 26

2.14. Evolución de la normativa en España . . . . . . . . . . . . . . 27

2.15. Curva española de tensión-tiempo que define el área del hueco de tensión en el punto de conexión a la red que debe ser soportado por la instalación. . . . . . . . . . . . . . . . . 28

2.16. Corriente reactiva admisible (generada o consumida), en función de la tensión en bornes. . . . . . . . . . . . . . . . . . . . 29

2.17. Curva italiana de tensión-tiempo que define el área del hueco de tensión en el punto de conexión a la red que debe ser soportado por la instalación. . . . . . . . . . . . . . . . . . 30

2.18. Curva propuesta de tensión-tiempo que define el área de la perturbación de tensión en el punto de conexión a red. Exceptuando el caso de cortocircuitos bifásicos aislados de tierra. . 31 
2.19. Curva propuesta de tensión-tiempo que define el área de la perturbación de tensión en el punto de conexión a red. Caso de cortocircuitos bifásicos aislados de tierra. . . . . . . . . . 32

2.20. Comparativa de requerimientos tensión-tiempo. Normativa actual y propuesta en estudio. . . . . . . . . . . . . . 32

2.21. Guía para los requerimientos de conexión: Curva límite de tensión para las plantas de generación en el punto de conexión

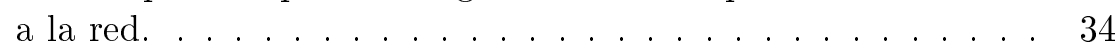

3.1. Hueco de tensión. . . . . . . . . . . . . . . . . . . . 38

3.2. Ejemplo de hueco rectangular. . . . . . . . . . . . . 42

3.3. Ejemplo de hueco no rectangular. . . . . . . . . . . . . . . . . . . . . . . . . . 43

3.4. Ventana deslizante. . . . . . . . . . . . . . . . . . 44

3.5. Huecos a diferentes niveles de tensión debidos a diferentes tipos de faltas [2]. . . . . . . . . . . . . . . . . . . 45

3.6. Representación de la trayectoria del vector $\mathrm{v}(\mathrm{t})$ sobre el plano

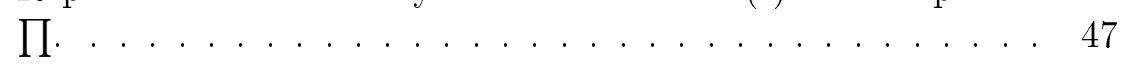

3.7. Cambio de base de los ejes $\alpha-\beta-\gamma$ a los ejes $d q 0$. . . . . . 47

3.8. Representación del vector espacial v en la nueva base $d q 0$. . . 48

3.9. Descomposición del vector espacial como suma de dos vectores de módulo y velocidad angular constantes [3]. . . . . . . . . . 49

3.10. Vector espacial para un hueco manofásico tipo B, en las fases a y c respectivamente $[3] . \ldots \ldots \ldots$

3.11. Ejemplo de hueco bifásico medido en un huerto solar fotovol-

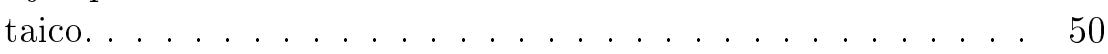

3.12. Ejemplo de hueco trifásico medido en un huerto solar fotovol-

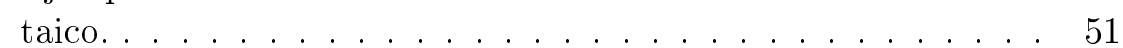

3.13. Ejemplo de hueco monofásico medido en una parque eólico. . 51

3.14. Ejemplo de hueco trifásico medido en un parque eólico. . . . . 51

3.15. Comparativa huecos de tensión. . . . . . . . . . . 53

3.16. Comparativa de perturbaciones reales frente a normativa de conexión española para instalaciones renovables dada en [4].] . 54

3.17. Curva española de tensión-tiempo que define el área del hueco de tensión en el punto de conexión a la red que debe ser soportado por la instalación. . . . . . . . . . . . . . 55

3.18. Requerimientos de conexionado para parques eólicos: Comparativa de las curvas del valor límite de tensión eficaz en el punto de conexión a la red [1]. . . . . . . . . . . . . . 55

3.19. Caracterización de perturbaciones mediante conjunto de longitudes. Aplicación a requerimientos del sistema eléctrico es-

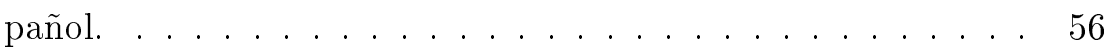


3.20. Caso práctico de identificación de longitudes. Comparativa ante requerimientos del sistema eléctrico español. . . . . . . . 57

4.1. Ejemplo gráfico de dispersión. . . . . . . . . . . . . . . . . . . 62

4.2. Gráfico de dispersión incluyendo el área de la perturbación. (Fuente: [5]). . . . . . . . . . . . . . . . . 62

4.3. Vista general de la planta FV y el analizador de calidad de potencia-Fluke 1760-. . . . . . . . . . . . . . . . . . 63

4.4. Resultados de campo en instalaciones FV: mapa de colores. . 66

4.5. Resultados de campo en parque eólico: mapa de colores. . . . 67

4.6. Resultados medidos en la planta FV: Propuesta de diagrama de dispersión para evaluar la severidad de hueco de tensión. . 69

4.7. Comparativa de los requerimientos para plantas FV en el punto de conexión con la red (Ejemplos de normativa europea) . 70

4.8. Resultados medidos en la planta FV: Comparativa con los requisitos de conexión. . . . . . . . . . . . . . . . . . . 71

4.9. Análisis de componentes. Resumen general. . . . . . . . . . . 73

4.10. Análisis de Componente Principal y K-means Clustering. Descripción General. . . . . . . . . . . . . . . . . . . . . . . . . . 77

4.11. Huecos de tensión en parque eólico. Caracterización por componentes $P_{1}$ y $P_{2}$. Identificación de grupos y patrones mediante $\mathrm{K}$-means Clustering. . . . . . . . . . . . . . . . . . . . 80

4.12. Huecos de tensión en parque eólico. Mapa de calor por caracterización de componentes $P_{1} C_{1} P_{2}$. . . . . . . . . . . . . . 82

4.13. Huecos de tensión en parque eólico. Comparativa de patrones de tensiones eficaces con requerimientos de conexión español. 83

4.14. Huecos de tensión en instalaciones FV. Caracterización por componentes $\mathrm{PC}_{1}$ y $\mathrm{PC}_{2}$. Identificación de grupos y patrones mediante K-means clustering. . . . . . . . . . . . . . . . . . . 85

4.15. Huecos de tensión en instalaciones FV. Mapa de calor por caracterización de componentes $\mathrm{PC}_{1}$ y $\mathrm{PC}_{2}$. . . . . . . . . . . . 85

4.16. Huecos de tensión en instalaciones FV. Comparativa de patrones de tensiones eficaces con requerimientos de conexión español. ....................... 87

5.1. Proposal of Grid-Code Requirements by applying $d q$ transformation: comparison with a collected disturbance. . . . . . . . 93 



\section{Índice de Tablas}

2.1. Potencia instalada y acumulada por fuentes de energía eólica y FV en todos los países europeos en 2012. . . . . . . . . . . . 18

2.2. Guía para los requerimientos de conexión: parámetros para tiempo y tensión dentro de los rangos definidos por la normativa. 34

3.1. Huecos a diferentes niveles de tensión debidos a diferentes tipos de faltas [2].]. . . . . . . . . . . . . . . . . . . . . . . . . 44

4.1. Resultados medidos en instalaciones FV: Tabla huecos de tensión. . . . . . . . . . . . . . . . . . 65

4.2. Resultados medidos en parque eólico: Tabla huecos de tensión. 65

4.3. Huecos de tensión en parque eólico. Distribución de variabilidad por componentes principales. . . . . . . . . . . . . . 78

4.4. Huecos de tensión en parque eólico. Coeficientes por componentes principales. . . . . . . . . . . . . . . . . 79

4.5. Huecos de tensión en parque eólico. Proceso K-means clustering: identificación de clusters y variabilidad de longitudes . . 81

4.6. Huecos de tensión en parque eólico. Proceso K-means clustering: identificación de clusters y parámetros principales de tensión y duración . . . . . . . . . . . . . . . . . . . . . . . . 81

4.7. Huecos de tensión en instalación FV. Distribución de variabilidad por componentes principales. . . . . . . . . . . . . . . 84

4.8. Huecos de tensíón en instalación FV. Coeficientes por componentes principales. . . . . . . . . . . . . . . . . . . 84

4.9. Huecos de tensión en instalaciones FV. Proceso K-means clustering: identificación de Clusters y variabilidad de longitudes. $\quad 86$

4.10. Huecos de tensión en instalaciones FV. Proceso K-means clustering: identificación de Clusters y parámetros principales de tensión y duración. . . . . . . . . . . . . . . . . . . . . . . . . 87 



\section{Capítulo 1}

\section{Aims and Motivations.}

\section{ABSTRACT:}

In this chapter, aims and motivations of this $\mathrm{PhD}$ dissertation are discussed by providing an outline of the problem to be addressed, and by justifying the interest of the topic.

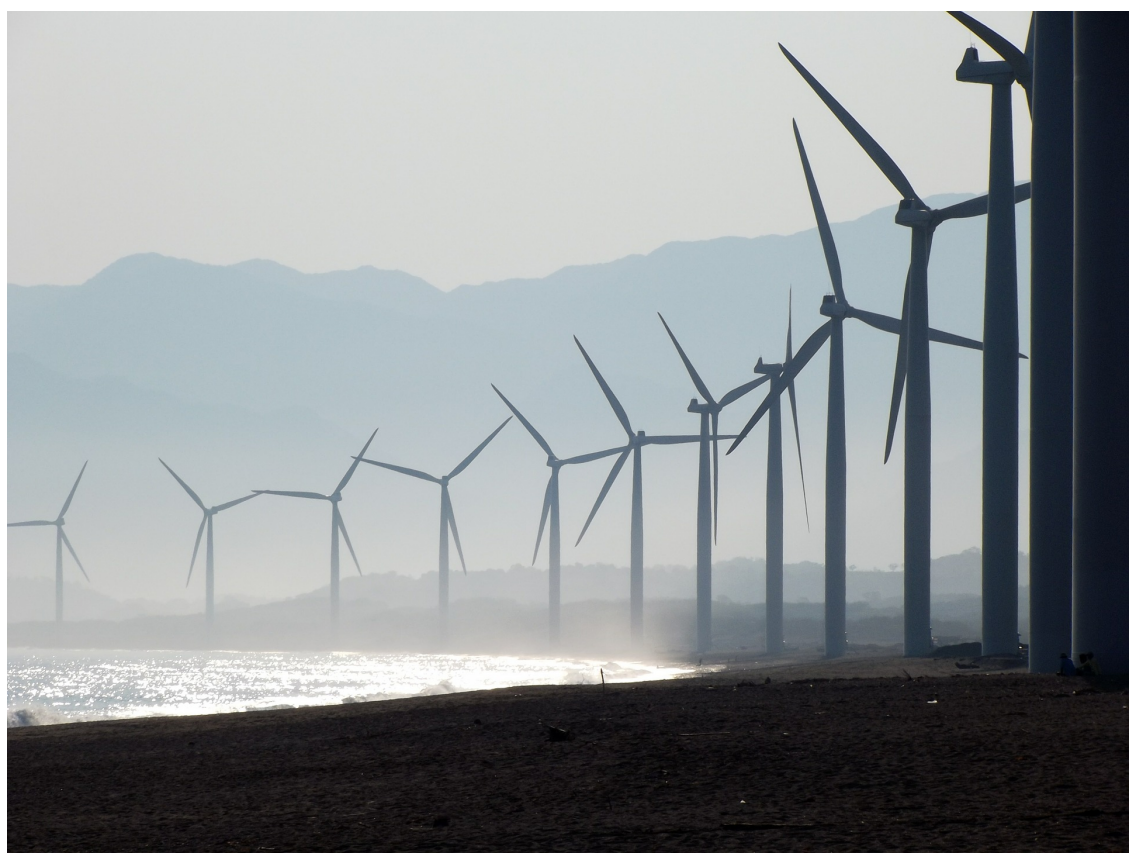

Wind farm - Bangui Ilocos North Philippines (Creative Commons Licence) 


\subsection{Aims.}

Traditionally, fossil fuels have been remarkable sources of primary energy in most countries, and they still continue to dominate growth in power generation. However, during the last decades, a remarkable number of goverments have been promoting policy principles and incentives to change this tendency, turning an efficient and renewable scenario into reality. Nevertheless, and despite the significant growth in lowcarbon sources of energy - shifting away from oil and coal towards natural gas and renewable sources-, fossil fuels currently remain dominant in the global energy mix. In this context, and aiming to reduce the fossil fuel dependence and foreign energy sources, electricity emerges as a sector where renewable energy policies and energy efficient initiatives are most likely to be implemented, since it can be generated from a great variety of fuels and technologies. Consequently, displacing fossilfired generation with renewable generation presents many desirable outcomes within the power sector, such as the reduction in pollution and $\mathrm{CO}_{2}$ emissions. The combination of all these factors has led to the increasing electricity generation by Renewable Energy Sources (RES). Indeed, some authors affirm that these factors are driving the push for more renewable energy integration [6], 7]. In these new and upcoming policy scenarios, electricity generation from renewables should nearly triple from 2010 to 2035, reaching $31 \%$ of total generation [8]. This rapid growth in the installed renewables must be integrated into the grid properly [9, 10]. With this proposal, current grid codes are requiring acceptable power quality of electric power systems under a sort of disturbances. The term Power Quality (PQ) includes the electromagnetic phenomena characterizing voltage and current waveforms [11. From the customer-side, voltage dips and supply interruptions are considered the most important PQ concerns [12,,[13],[14],[15],[16],[17,,[18]. These disturbances must be also supported by generator units connected to grid, including RES installations. Actually, and as the range of RES technologies achieving significant level penetrations, the interest of analyzing their potential impacts on the electrical distribution grid as well as the possibilities to offer ancillary services from these renewable resources is increasing considerably [19].

Under this framework, where the connection and continuity requirements for RES is a current topic of insterest and some countries have developed specific grid-codes for the connection of wind and PV to the power grid, the present $\mathrm{PhD}$ document involves the following objectives:

- Analysis of current grid-codes in different European countries for RES submitted to voltage dips. Comparison of requirements and discussion about new drafts promoting by ENTSO-E to give general rules for new generators units connected to the grid. 
- Characterization of voltage dip severity by including as additional parameter the time interval around the minimum voltage value - residual rms voltage- .

- Definition of a set of segment lenghts related with partial time intervals and rms-voltage trajectories to summarize the corresponding evolution of the rms-voltage values along the event.

- Application of Principal Component Analysis and K-means clustering to identify patterns and groups of voltage dips among a large dataset of events. This identification of patterns and similarities of voltage dips reduce significantly the number of events to be represented.

The identification of patterns for the collected voltage dips through a representative set of rms-voltage profiles allows a suitable comparison with current grid code requirements. Indeed, most current requirements are given by means of rms-voltage trajectories. Consequently, an averaged estimation of the severity of disturbances can be deduced from a simple comparison between patterns and requirements, in terms of rms-voltage limitations required by the operational procedures. This proposed methodology constitutes a novel and convenient manner to characterize and classify a large number of disturbances, when a direct comparison with requirements or rules is not operative in terms of functionality and operation for both wind farm operators and Transmission Systems Operators.

\subsection{Justification of the Interest and Motivations.}

As the range of grid-connected Renewable Energy Sources (RES) technologies is achieving significant penetration levels, the interest of analyzing the potential impacts of RES on the electrical distribution grid is considerably increasing [20, [21]. Moreover, the possibilities of offering ancillary services from these renewable resources is nowadays a topic of interest [21], Examples of recent contributions can be found in the literature: [22] is focused on PV solar production for residential applications, or [23] analyzes intermittent wind resources. Consequently, and aiming at maintaining continuity and security on the electric supply-side, some countries have developed specific Grid-Code requirements for the connection of RES to the grid, avoiding undesired disconnection under the presence of disturbances - mainly voltage dips - . These requirements have mainly focused on the wind farms connected to the grid [24], due to their major presence in current power systems. As a matter of fact, the annual wind power installations in EU have increased steadily over the past 12 years from $3.5 \mathrm{GW}$ in 2000 to $11.2 \mathrm{GW}$ in 2013 , with an average annual growth rate of $11 \%$ [25]. 
Grid-Code requirements have been usually expressed in terms of rmsvoltage against time characteristic. In all cases, if rms-voltage trajectories remain above the minimum allowed voltage limits, the generating units including renewables - shall remain transiently stable and connected to the system, without tripping for a close-up solid three-phase short circuit fault or any unbalanced short circuit fault on the transmission system [26]. However, these rules vary significantly among countries, depending on the renewable technology penetration level as well as on the robustness of the national or regional power system. Contributions including both characterization and classification techniques for power disturbances can be found [27], [2], 28], but only a few contributions present power quality surveys of renewable power installations [29], 30] 31] 32]. Moreover, there is a lack of works addressing the characterization and clustering of a group of real voltage dips with the objective of comparing with current requirements, and estimating how severe and close to the rms-voltage limits the collected events are. Representations usually reduce the analysis of events in terms of two main characteristics: residual rms-voltage vs time duration. These studies imply an important simplification of disturbances with the corresponding loss of information. Actually, and apart from the residual rms-voltage, the severity of the faults is highly dependent on the voltage evolution along the disturbance and the point-on-wave initiation [33]. Therefore, and spite comparison based on global rms-voltage dip trajectory vs time initially appears to be appropriate, this approach presents several limitations: (i) it can be suitable for a reduced number of events, but more difficult to handle if the number of event to be analyzed is significant; (ii) it doesn't provide a systematic characterization in terms of voltage dip severity and proximity to the rms-voltage limits. To overcome these drawbacks and offer an alternative solution, the present Thesis describes and evaluates a novel characterization of voltage dips based a set of lengths to identify clearly the rms-voltage trajectories along the disturbances. Additionally, Principal Components Analysis and a K-means clustering process have been applied to classify and identify distributions and tendencies of a large amount of events, allowing a visual comparison with current grid-code requirements in terms of rms-voltage profiles. The proposed methodology is evaluated using real disturbances collected in Spanish wind farm and PV installations along several field-measurement campaigns. The observed voltage dips are characterized and clustered, and the average rms-voltage patterns are then compared to the Spanish current Grid-Code requirements, allowing for a global estimation of the severity of the collected events.

The developments of this work are in line with two pre-doctoral periods carried out by the candidate: University of Aalborg (Denmark) and University of Coimbra (Portugal). Both experiences were focused on the analysis of european requirements and rules for renewable resources connected to the 
grid under voltage dips. Actually, these periods provide added value to this $\mathrm{PhD}$ document, since they allowed the candidate a relevant exchange of information ín situ'regarding current and upcoming rules for generation units connected to the grid, as well as a detailed study of renewables and their real integration into different power systems. In reference to the statistical and clustering analysis to identity patterns and more likely rms-voltage trajectories from a set of collected disturbances, these studies have been carried out in collaboration with the Dept. of Applied Mathematics and Statistics (Universidad Politécnica de Cartagena, Spain). Specifically with the Prof. M. Kessler, researcher with an extensive international career in applied statistics and co-author of relevant journal papers in this field.

From the point of view of the research group, the fulfilment of this thesis is in line with different previous contributions in the field of voltage dips and sensitive analysis of loads under perturbances. These approaches have been developed through different research projects financially supported by regional and national goverments:

- Electrical and Mechanical Modeling of wind turbines based on DFIG and PMSG solutions. An approach for integration into power systems (Ref. ENE2009-13106). From 2009 to 2012

- Analysis and simulation of new requirements for wind farm regulation. Integration as ancillary services into power systems with high wind power penetration (Ref. ENE2012-34603). From 2013 to 2015.

The rest of the document is structured as follows: Chapter 2 discusses about the integration of renewable energy sources into power systems during the last decade, as well as their relevant penetration level from the supplyside mainly due to the presence of wind farms and PV power plants. A description in detail of grid-codes and current requirements for generation units connected to the grid under the presence of disturbances is included as well. Chapter 3 gives a detailed analysis of previous characterization approaches for voltage dips, and introduces the definition of voltage dip severity by considering not only the residual rms-voltage value and the total duration of the event, but also the marginal time interval around this minimum rms-voltage. The introduction of this additional parameter leads to propose a set of segment lengths with the aim of characterizing in detail the trajectory of the rms-voltage along the disturbance. This proposal is applied on current Spanish grid-code requirements and on real events collected during field-measurement campaigns. Chapter 3.4 describes and evaluates a novel characterization process to visualize and classify large amount of voltage dips. From the set of lengths proposed to characterize the rms-voltage evolution along the disturbance, Principal Component Analysis and K-means clustering are applied to identify rms-voltage patterns and propose a reduced number of representative rms-voltage profiles from the initial collected 
disturbances. The corresponding reduced group of rms-voltage profiles offers a useful tool to classify a large amount of disturbances allowing a visual and graphical representation of their evolution along the event, aspects that are not previously considered in the specific literature. Finally, contributions and possible future work are given in Chapter 5 . 


\section{Capítulo 2}

\section{Normativa y Requerimientos de Conexión de Fuentes Renovables ante Perturbaciones.}

\footnotetext{
Resumen: La presencia de generación renovable en los sistemas eléctricos ha aumentado considerablemente en la última década, sobre todo alentada por el desarrollo de soluciones tecnológicas viables y por propuestas políticas de integración de recursos propios como vía para disminuir la dependencia energética con terceros países. En el caso de Europa, esta incorporación ha venido impulsada principalmente por el recurso eólico y el solar fotovoltaico. Como consecuencia de estas modificaciones sufridas por el mix de generación, se han propuesto una serie de cambios en los procedimientos de conexión a red de instalaciones renovables, principalmente los requerimientos que tienen que ver con conexión y comportamiento ante perturbaciones. En este escenario, este capítulo analiza tanto la evolución de la integración del recurso eólico y fotovoltaico en el sistema eléctrico europeo como los requisitos actuales de comportamiento de estos sistemas ante la presencia de huecos de tensión. Del mismo modo, se incluye una detallada discusión del último borrador publicado por el operador del sistema español Red Eléctrica Española (REE), así como las diferentes iniciativas y requerimientos para fuentes renovables ante la presencia de perturbaciones promovidas por el grupo de operadores de sistemas eléctricos europeos (ENTSO-E) con el fin de establecer pautas de comportamiento comunes en los diferentes países
} 


\subsection{Introducción.}

Los combustibles fósiles (petróleo, gas natural y carbón) han sido la principal fuente de energía primaria en el mundo, incluso hoy en día siguen constituyendo un pilar básico en el mix energético actual. Así lo corrobora el hecho de que éstos representen en torno al $80 \%$ del suministro total de energía primaria, un valor que se ha mantenido prácticamente invariable en los últimos veinticinco años [8]. Esta importante vinculación de la demanda de energía con fuentes basadas en combustibles fósiles ha llevado aparejada una serie de implicaciones, tanto desde el punto de vista de dependencia energética con terceros países como desde el punto de vista de consecuencias medioambientales y de cambio climático. Como ejemplos, durante el 2012, en el caso de Italia, la dependencia energética actual se situó en el 80,8\%, la de Portugal en el 79,5\%, la de España en el 73,3\% y la dependencia energética actual alemana es del $61,1 \%$. Esto contrasta con Dinamarca que es el único país europeo que exporta energía, teniendo un ratio de dependencia negativo, del $-3,4 \%$, asimismo tiene como objetivo cubrir el $50 \%$ de la demanda energética mediante energías renovables (EERR) para el 2035, pretendiendo llegar al $100 \%$ de la generación mediante fuentes de energías renonables (FER) para el año 2050. Además, este tipo de fuentes de naturaleza no renovables están jugando un papel más que destacable en el incremento de emisiones de $\mathrm{CO}_{2}$. De hecho, los países desarrollados están promoviendo esfuerzos de reducción de emisiones de $\mathrm{CO}_{2}$ con el fin de evitar los efectos no deseados derivados del cambio climático. Las políticas de reducción de emisiones pueden lograrse mediante la aplicación de diferentes iniciativas, entre las que destacan la introducción de cambios en las fuentes de energía primaria, la promoción y difusión de políticas de eficiencia energética, o las mejoras de los ciclos de vida en sectores como el industrial o el terciario. Como consecuencia de esta amalgama de factores, se ha producido en la Unión Europea una disminución del $8 \%$ entre 2006 y 2012 de consumo de energía primaria [34], incluyendo en este estudio los 28 estados miembros. A modo de ejemplo, esta reducción ha estado en torno al 15,2\% en Portugal, $14 \%$ en Dinamarca, $12 \%$ en España e Italia, y el 9,2\% en Alemania. Hay que resaltar que muchos autores vinculan que este descenso en el consumo energético a la crisis económica que hay en Europa, ya que el sector industrial, que es uno de los grandes consumidores de energía eléctrica, se ha mermado considerablemente, provocando con ello un menor consumo.

En este contexto, y con el objetivo principal de reducir la dependencia energética de los combustibles fósiles y de otros países, la electricidad se postula como un sector donde las políticas de energías renovables y las iniciativas sobre energía eficiente tienen más probabilidades de ser implementadas, ya que puede ser generada a partir de una gran variedad de combustibles y/o tecnologías. Por otra parte, la electricidad, como energía final, también se 
considera como una forma adicional de distanciarse de la utilización de los combustibles fósiles: alrededor de $75 \%$ de la energía consumida por el sector residencial y comercial es la electricidad y en el sector industrial el $35 \%$ del consumo de energía es electricidad [35]. En consecuencia, el desplazamiento de la generación con combustibles fósiles por la generación mediante energías renovables presenta muchos resultados deseables en el sector de la energía, tales como la reducción de la contaminación y las emisiones de $\mathrm{CO}_{2}$.

En cuanto a la generación de energía eléctrica, los cambios más significativos se han producido como consecuencia de la incorporación de nuevas soluciones basadas en fuentes renovables. De hecho, la energía eléctrica se ha obtenido a partir de una amplia gama de diversas tecnologías, cada una de ellas presentando una serie de ventajas y problemáticas de implementación y mantenimiento, como son las fuentes nuclear, hidroeléctrica, eólica, solar...[36]. Así pues, esta característica de flexibilidad en cuanto a la elección de fuente primaria ofrece oportunidades extraordinarias dentro del sector eléctrico para incluir soluciones basadas en fuentes de naturaleza renovable que atenúen y reduzcan las emisiones de gases de efecto invernadero así como la dependencia energética con terceros países. Es más, si se analiza este sector con cierta perspectiva temporal, puede afirmarse que la electricidad ha jugado una parte creciente en el porcentaje de fuentes de energía final durante las últimas décadas, pasando de $9,4 \%$ en 1973 al 17,7\% en 2010; mientras los derivados del petróleo se han mantenido en un estrecho rango casi constante, en torno al $45 \%$ [37]. Así pues, en este escenario de crecimiento de la demanda eléctrica y de presencia relevante en sectores clave como el industrial o el terciario, la sustitución de la generación de combustible fósil por la generación basada en fuentes renovables presenta una serie de ventajas y consecuencias medioambientales que no han pasado desapercibidas a nivel internacional, destacándose la importante labor que este tipo de incorporaciones desarrollaría principalmente en las reducciones de emisiones de $\mathrm{CO}_{2}$. La combinación de todos estos factores ha llevado al aumento de la producción de electricidad mediante FER, siendo apoyadas por políticas energéticas sostenibles promovidas por los gobiernos de los países más desarrollados. En este escenario de políticas energéticas, la generación de electricidad a partir de energías renovables espera triplicarse desde 2010 hasta 2035, llegando al $31 \%$ de la generación eléctrica total [38]. Es más, algunos países como es el caso de Dinamarca, han promovido iniciativas e incentivos para cubrir en 2050 el $100 \%$ de necesidades eléctricas del país a través de fuentes renovables. Además, la desregularización (privatización y liberalización) del sector eléctrico ha invitado a las entidades de generación y proveedores a satisfacer la siempre creciente demanda de electricidad [39].

Las fuentes renovables más extendidas en la generación de energía eléctrica, han sido las instalaciones basadas en fuentes eólica y fotovoltaica (FV), con más de 115,4 y $80 \mathrm{GW}$ de potencia instalada en Europa respectivamente 


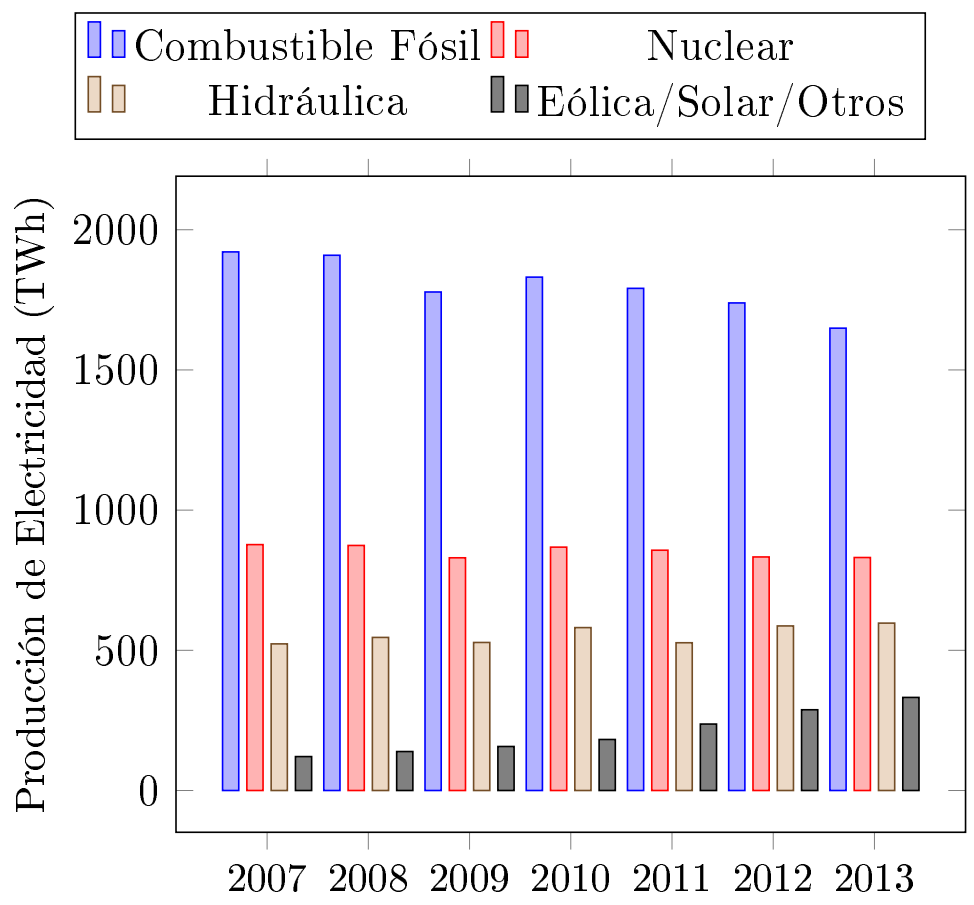

Figura 2.1: Comparativa de producción en función del tipo de combustible en Europa.

[40]. En cuanto a las fuentes renovables alternativas (hidráulica, biomasa, residuos, termo-solar, geotérmica y mareomotriz) también han incrementado su potencia instalada en la última década, pero en menor medida que los recursos eólico y fotovoltaico. La figura 2.1 resume la producción de electricidad en Europa por tipo de combustible para el período 2000-2013 [41].

Esta creciente influencia del sector renovable y su alta penetración en los sistemas eléctricos (fundamentalmente de origen eólico) lleva aparejada la necesidad de realizar estudios de integración de este tipo de tecnologías en el diseño y operación de los sistemas eléctricos [42], [43], [44, [45], [46], [47. Así, aspectos fundamentales tales como la variabilidad de la generación basada en fuentes solares y eólicas han sido recientemente motivo de estudio, al igual que la propia variabilidad asociada a la demanda eléctrica [48], 49], [50]. Estos cambios en el uso de fuentes convencionales por FER ha suscitado una gran preocupación, dado el impacto que tiene de las FER en la red con 
su generación intermitente y/o potencia fluctuante.

En paralelo con la integración de fuentes renovables en el sector eléctrico, y atendiendo a la cuota cada vez mayor tanto de capacidad instalada de FER como de cobertura de la demanda, ha surgido la necesidad de evaluar y analizar las implicaciones que este tipo de fuentes lleva consigo sobre el sistema eléctrico en su conjunto, [20]. A este respecto, y tal como se ha comentado anteriormente, el crecimiento y la conexión al sistema eléctrico de un gran número de puntos de generación puede tener implicaciones tanto en la fiabilidad del sistema como en la calidad de suministro [51]. De hecho, y con el fin de asegurar la continuidad de suministro y la seguridad y fiabilidad del sistema, algunos países han establecido requisitos específicos para la conexión de los parques eólicos y plantas FV a la red eléctrica [52. Estos requisitos de permanencia en conexión de este tipo de fuentes ha implicado una ampliación de las características requeridas a parques eólicos e instalaciones FV, imponiendo un comportamiento no sólo a nivel de potencia activa a lo largo de la perturbación sino también de requerimientos de reactiva [48].

En este marco actual donde la integración de las tecnologías basadas en renovables suponen al mismo tiempo una vía comúnmente aceptada de reducción de dependencia de fuentes externas como una alternativa para reducir las emisiones de gases de efecto invernadero, este capítulo se describe y discute las propuestas actuales y los requisitos técnicos exigidos para instalaciones de fuentes renovables. Se comienza analizando la situación energética de Europa de la energía FV y la energía eólica, donde se expone como ha ido creciendo el número de instalaciones de estas energías, cual es la situación actual y las propuestas para el año 2020. En el siguiente punto se muestran la normativa vigente que abarcan los requisitos técnicos de conexión para las instalaciones eólicas y FV, en distintos países europeos. Por último, se comentan algunas propuestas de normativa de conexionado, tanto a nivel europeo como la de algunos países.

\subsection{El Sector Eólico y Fotovoltaico.}

Durante las últimas décadas se ha fomentado las instalaciones de FER. La potencia instalada en el año 2000 fue alrededor de 3,6 GW, representando el $22,4 \%$ de las nuevas plantas de generación que se instalaron ese año. En 2013, esta capacidad se incrementó a 47,4 GW, contabilizando más del $72 \%$ de las nuevas instalaciones. Como ya se ha comentado anteriormente, dentro de las fuentes de energía renovables, la energía eólica y FV son las tecnologías más extendidas, con más de 115,4 y $80 \mathrm{GW}$ instalados respectivamente. De acuerdo con [53], ninguna otra tecnología ha alcanzado o alcanzará la madurez suficiente para desafiar a las fuentes FV, eólica o de gas en los cinco años próximos. No obstante, el resto de tecnologías renovables (hidroeléctrica, biomasa, residuos, plantas de concentración solar (en inglés concentrated 


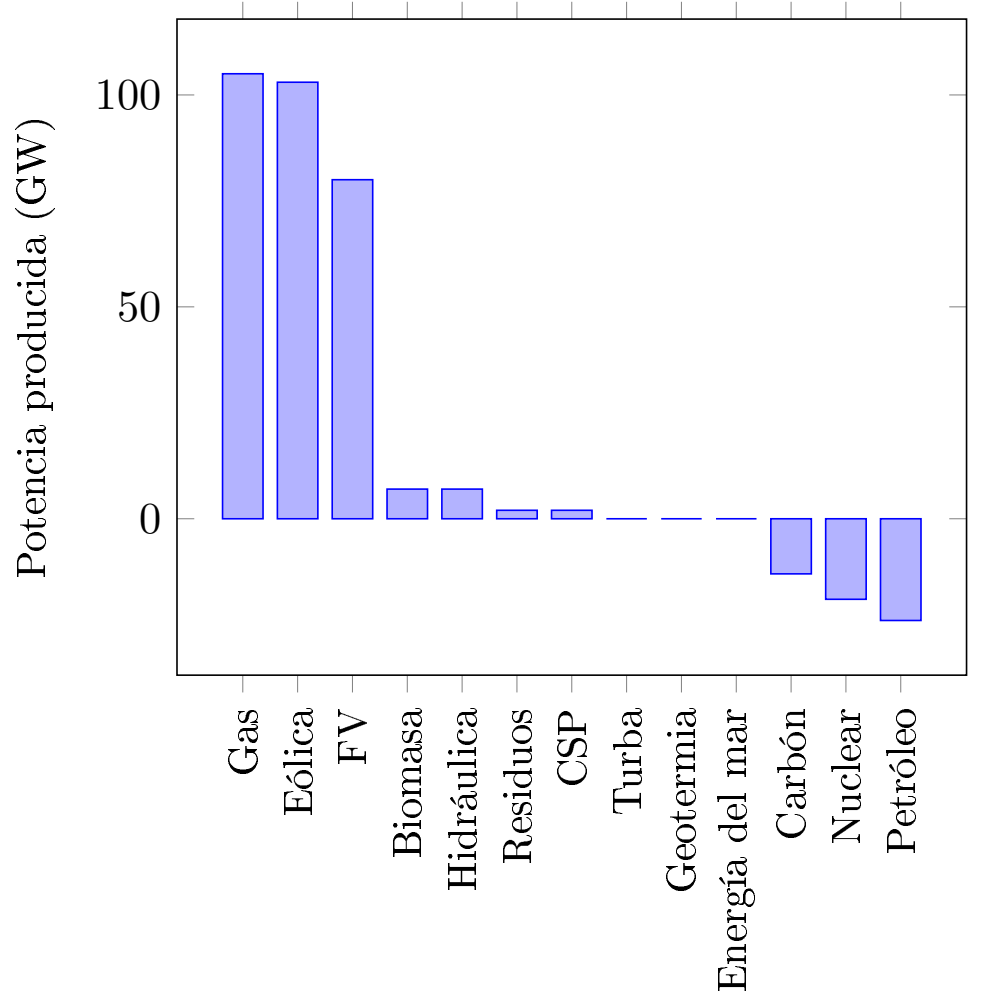

Figura 2.2: Potencia generada neta durante 2000-2013.

solar power, CSP), geotérmica y energías oceánicas) también han incrementado su capacidad instalada en la última década, pero en menor medida que las instalaciones eólicas y FV. La figura 2.2 muestra la capacidad neta de generación incorporada dentro del período 2007-2013 [54]. Durante los últimos tres años, las plantas $\mathrm{FV}$ han sido la fuente de generación que más capacidad instalada ha tenido en Europa. De hecho, la potencia instalada correspondiente a plantas FV en 2013 fue de más de 11 GW (31\% de la capacidad total), similar a la de los parques eólicos con alrededor de 11,2 GW $(31 \%)$ y gas con $10,5 \mathrm{GW}(23 \%)$. El resto de los recursos, tanto renovables como instalaciones convencionales, están lejos de esos valores. La figura 2.3 muestra la potencia instalada en Europa a lo largo del año 2013 [54].

En Europa las instalaciones de energía eólica se han ido incrementado constantemente en los últimos 13 años, pasando de 3,5 GW en el 2000 a 11,2 GW en 2013, con un porcentaje de crecimiento medio anual del $10 \%$. Como se muestra en la figura 2.4 actualmente hay instalados un total de 117,3 GW en la Unión Europea (UE), con un incremento del $10 \%$ en potencia acumulada con respecto al año anterior. La potencia eólica instalada en el 2013 puede llegar a producir 257 TWh de electricidad, suficiente para cubrir 


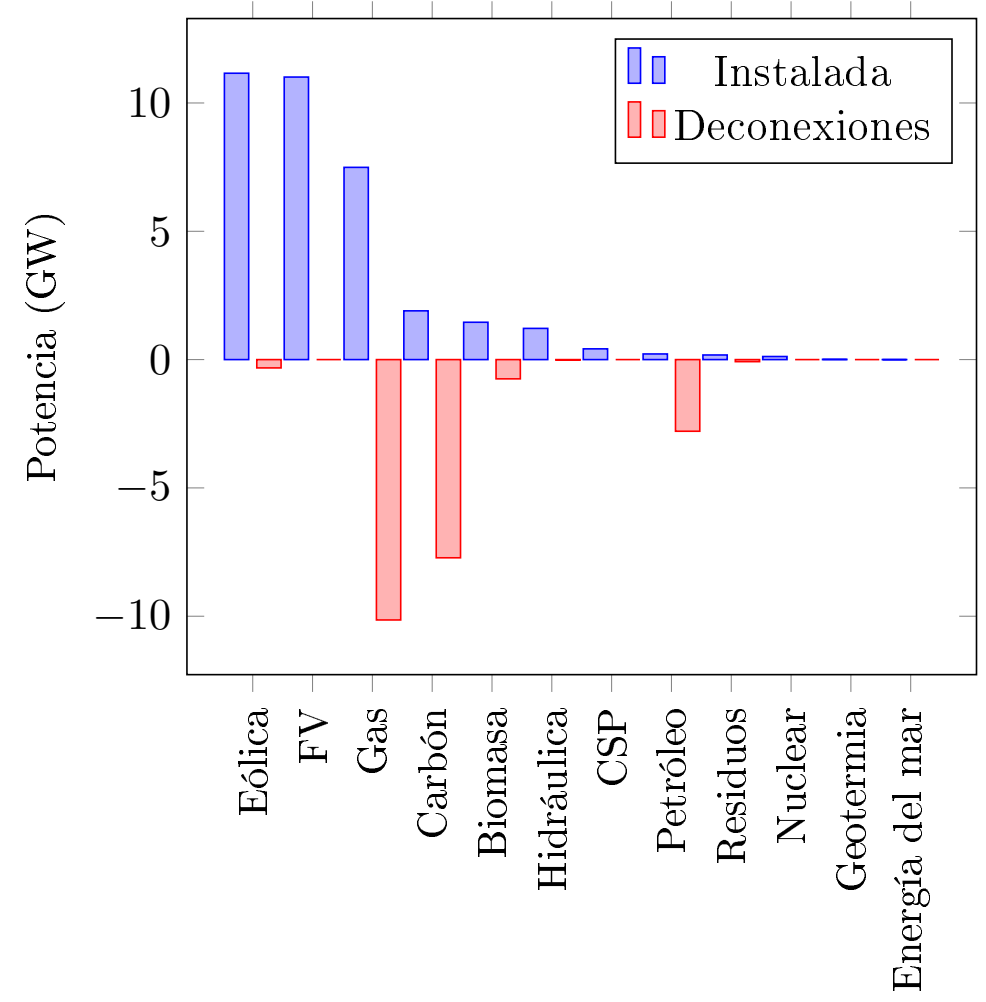

Figura 2.3: Nueva potencia instalada y potencia desconectada en 2013. 


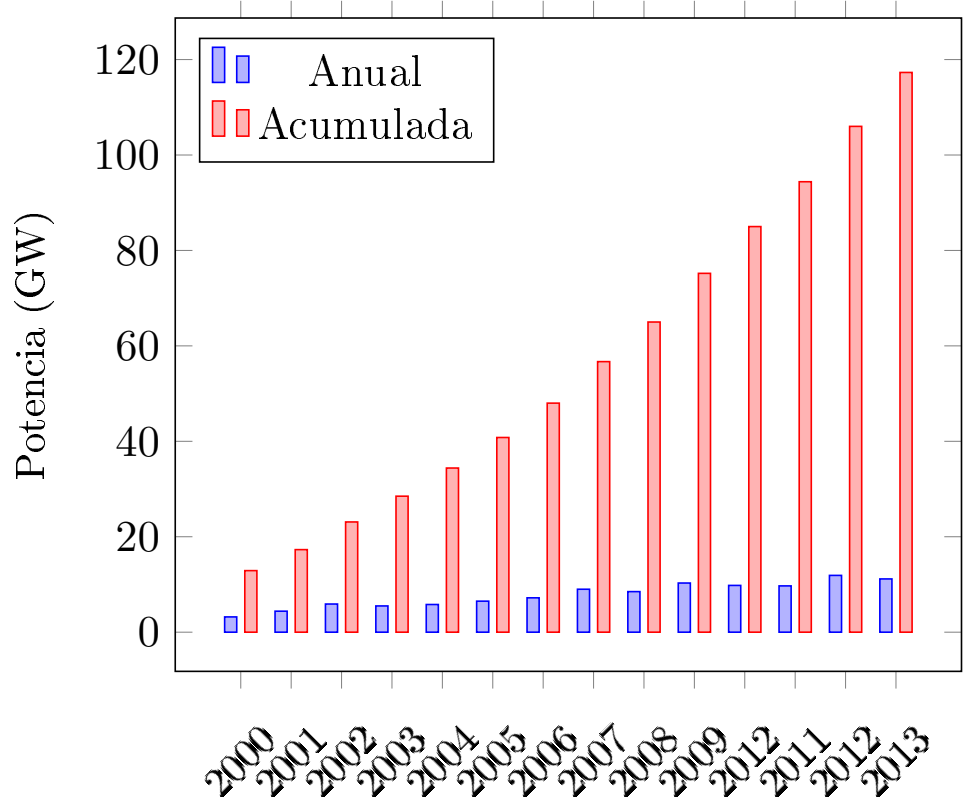

Figura 2.4: Potencia eólica instalada en la UE durante 2000-2013.

el $8 \%$ del consumo de electricidad en la UE.

La energía eólica se ha visto afectada por la crisis económica que barre Europa. Esta crisis ha provocado una incertidumbre regulatoria y política presentando durante el 2013 un impacto negativo sobre el mercado. Aún dentro de esta situación, la tecnología eólica es la que más se ha instalado en 2013: con un $32 \%$ de la potencia total instalada - cinco veces más que los años anteriores, [54]. El grueso de esta cantidad de energía es proporcionada principalmente por cuatro países, Alemania, España, Reino Unido e Italia. Además, Europa cuenta con 23 países con más de $1 \mathrm{GW}$ instalados, ocho de los cuales superan los $4 \mathrm{GW}$.

En cuanto a la generación de energía FV, su crecimiento está siendo considerablemente alto en los últimos años: a finales del 2009, la potencia FV instalada acumulada en Europa se acercaba a 17 GW y un año después era de $30 \mathrm{GW}$. Sin embargo, esta fuente de energía ha sufrido recientemente un descenso significantivo, en términos de potencia instalada. En 2013, se instalaron $11 \mathrm{GW}$ menos que el año anterior, que se instalaron 17,7 GW muy alejado de cifras de 2011, donde la potencia instalada fue 22,4 GW [55]. A pesar de este descenso de potencia instalada, la energía FV sigue siendo la tercera fuente de energía más importante después de la energía hidráulica y eólica. Durante los últimos tres años la energía FV ha sido una de las dos fuentes de energía más instaladas en la Unión Europea. En 2012, se instalaron más de $70 \mathrm{GW}$ — una cantidad capaz de suministrar al menos 77 TWh de electricidad al año-. La figura 2.5 muestra cómo ha evolucionado 


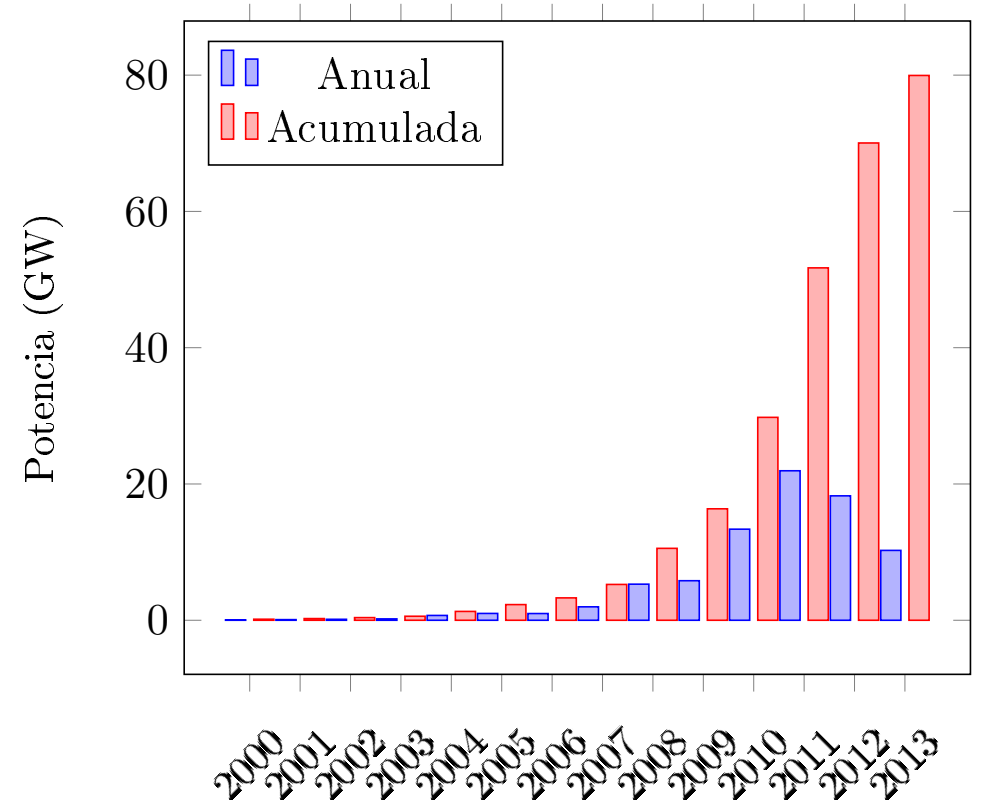

Figura 2.5: Potencia FV instalada en Europa durante 2000-2013.

la capacidad de potencia FV [55]. En este momento, la generación de energía FV es una parte no despreciable del mix eléctrico en Europa, cubriendo el $3 \%$ de la demanda en la UE y alrededor del $6 \%$ durante los periodos de máxima demanda.

El sector de la energía FV se ha visto muy perjudicado por la crisis económica que hay en Europa. Muchos países europeos (Alemania, Italia, Bélgica, Francia y España) han tomado decisiones políticas con el fin de reducir los incentivos destinados a este sector, provocando con ello un descenso en el mercado de la energía. Los casos más llamativos los han presentado países como España y la República Checa que han tenido crecimientos muy notables seguidos de periodos de instalación casi despreciable. El auge español se puede ver como la consecuencia de la primera fase de la crisis, pero también como la estabilización después de la experiencia de la expansión FV en 2008. En 2010, la energía FV también experimentó un alto crecimiento, con las nuevas instalaciones, principalmente, de países como Alemania, Italia y República Checa. El año 2011 ha sido el año con más potencia instalada en Europa, los mayores contribuyentes a este crecimiento se deben a un alto nivel de FV instalada en Italia y las instalaciones alemanas. En 2012, la potencia instalada empieza a decaer, se mantiene gracias a que Alemania presentó un récord en potencia instalada, con el $44 \%$ de las nuevas plantas FV introducidas en el mercado, e Italia, con el $20 \%$, mantiene constante su crecimiento. No obstante, en 2013, estos países que han mantenido a Europa líder del mercado mundial, empiezan a mostrar un declive en sus instalacio- 


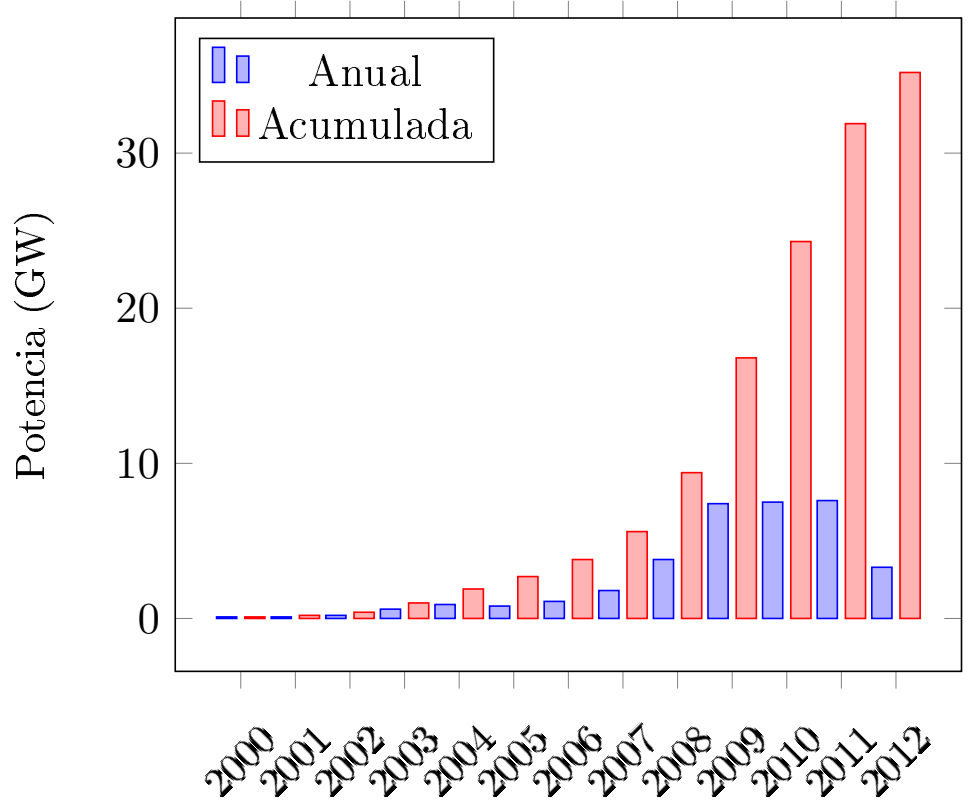

Figura 2.6: Potencia FV instalada en Alemania durante 2000-2013.

nes FV.

La evolución de los mercados de los países que más han ayudado a la consolidación de la FV se puede ver en las figuras 2.6, 2.7 y 2.8, [56]. En términos de consumo de electricidad, en Italia, más del $5 \%$ de electricidad se abastece de sistemas fotovoltaicos conectados a la red en 2011. En Alemania es alrededor del 4\%, y en otros países como en España o Bélgica están incrementando la cobertura de la energía demandada [53. La tabla 2.1 resume la nueva potencia instalada y acumulada para energía eólica y FV en los países europeos [25], [57]. La desaceleración sufrida por los sectores eólico y FV ha estado influenciada tanto por la crisis financiera como por las diversas realidades sociales y políticas de cada país. Así queda reflejado en la distinta evolución de estos mercados al analizar y comparar diferentes sistemas eléctricos y mix de generación.

A pesar de la incertidumbre actual respecto de la evolución de la economía mundial y su recuperación en el futuro, la demanda energética mundial sigue creciendo a un ritmo considerable: un 5\% en el año 2010 [58]. En escenarios elaborados por la Agencia Internacional de la Energía para el año 2035, la demanda energética mundial aumentaría un tercio, básicamente en países que no pertenecen a la Organización para la Cooperación y el Desarrollo Económicos (OCDE). El problema de la integración de las EERR en el sistema eléctrico es, en este momento, un tema de interés de los países europeos. En el año 2007 el Consejo Europeo aprobó el objetivo obligatorio de alcanzar una cuota del $20 \%$ de energía procedente de FER en el consu- 


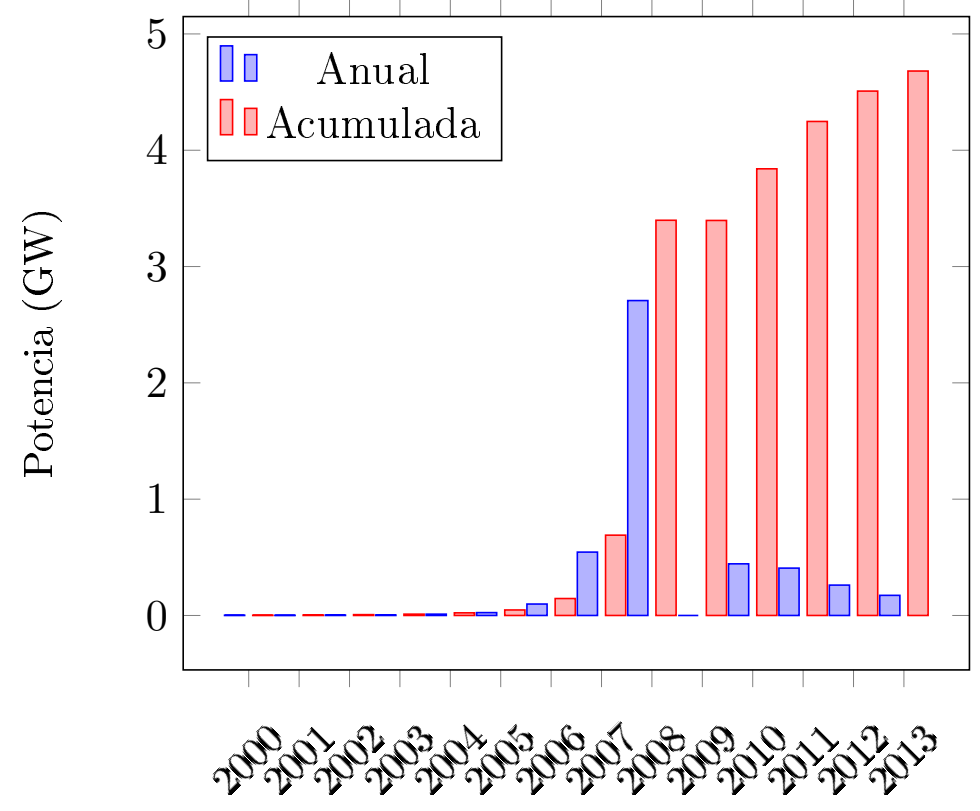

Figura 2.7: Potencia FV instalada en España durante 2000-2013.

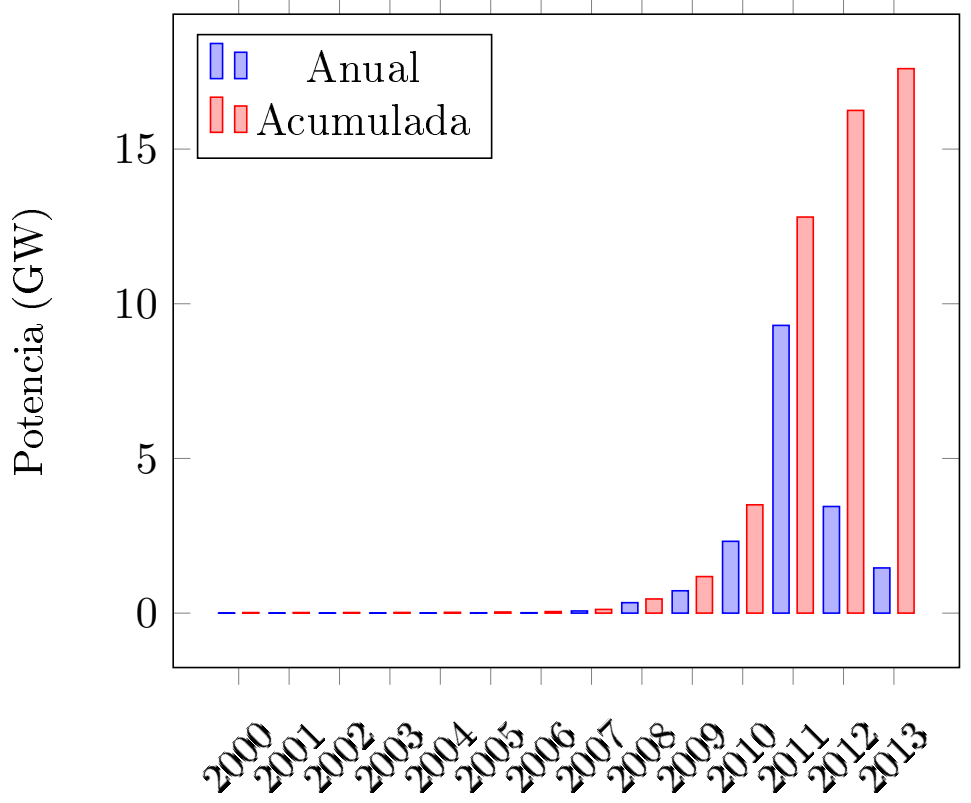

Figura 2.8: Potencia FV instalada en Italia durante 2008-2013. 


\begin{tabular}{|l|c|c|c|c|}
\hline & $\begin{array}{c}\text { Eólica } \\
\text { Instalada 2012 }\end{array}$ & $\begin{array}{c}\text { Eólica } \\
\text { Acumulada 2012 }\end{array}$ & $\begin{array}{c}\text { FV } \\
\text { Instalada 2012 }\end{array}$ & $\begin{array}{c}\text { FV } \\
\text { Acumulada 2012 }\end{array}$ \\
\hline Austria & 0.296 & 1.378 & 0.230 & 0.418 \\
Belgica & 0.297 & 1.375 & 0.599 & 2.650 \\
Bulgaria & 0.168 & 0.684 & 0.767 & 0.903 \\
Croacia & 0.052 & 0.180 & 0 & 0 \\
Cipre & 0.013 & 0.147 & 0 & 0.009 \\
República Checa & 0.044 & 0.260 & 0.113 & 2.072 \\
Dinamarca & 0.211 & 3.956 & 0.378 & 0.394 \\
Estonia & 0.086 & 0.269 & 0 & 0 \\
Finlandia & 0.089 & 0.288 & 0 & 0.001 \\
Francia & 0.757 & 7.564 & 1.079 & 4.003 \\
Alemania & 2.415 & 31.308 & 7.604 & 32.411 \\
Grecia & 0.117 & 1.749 & 0.912 & 1.536 \\
Hungría & 0 & 0.329 & 0 & 0.004 \\
Irlanda & 0.125 & 1.738 & 0 & 0.003 \\
Italia & 1.273 & 8.144 & 3.438 & 16.361 \\
Letonia & 0.021 & 0.068 & 0 & 0.001 \\
Lituania & 0.046 & 0.225 & 0.006 & 0.006 \\
Luxemburgo & 0 & 0.045 & 0 & 0.030 \\
Malta & 0 & 0 & 0 & 0.012 \\
Holanda & 0.119 & 2.391 & 0.125 & 0.266 \\
Noruega & 0.166 & 0.703 & 0 & 0 \\
Polonia & 0.880 & 2.497 & 0.004 & 0.007 \\
Portugal & 0.145 & 4.525 & 0.049 & 0.244 \\
Rumanía & 0.923 & 1.905 & 0.026 & 0.030 \\
Eslovaquia & 0 & 0.003 & 0.015 & 0.523 \\
Eslovenia & 0 & 0 & 0.117 & 0.198 \\
España & 1.122 & 22.796 & 0.276 & 5.166 \\
Suecia & 0.846 & 3.745 & 0.008 & 0.019 \\
Suiza & 0.004 & 0.050 & 0.200 & 0.416 \\
Turquía & 2.312 & 0.002 & 0.009 \\
Ucrania & 0.506 & 0.276 & 0.182 & 0.373 \\
Reino Unido & 0.125 & 0.445 & & 1.829 \\
\hline
\end{tabular}

Tabla 2.1: Potencia instalada y acumulada por fuentes de energía eólica y FV en todos los países europeos en 2012. 


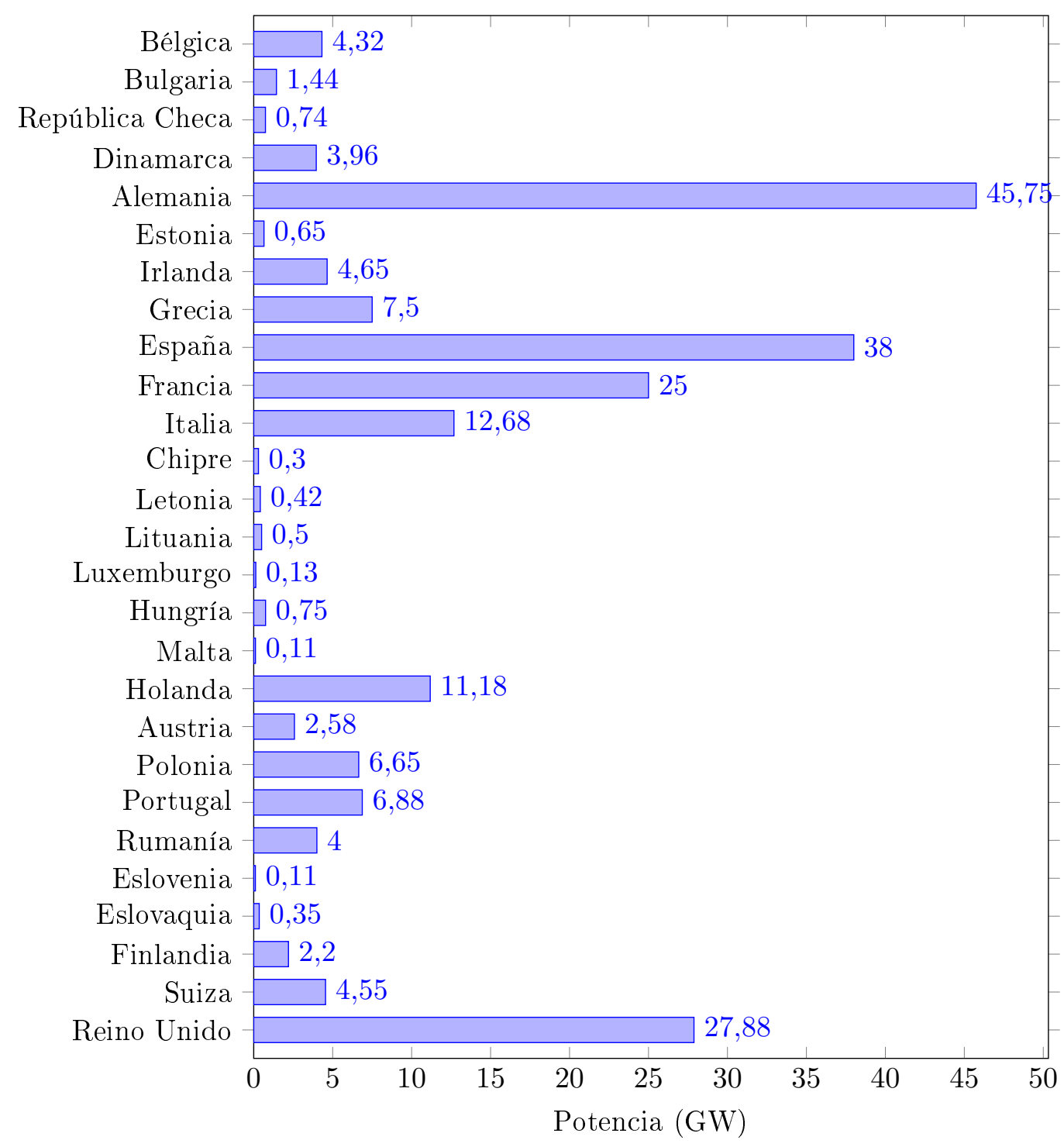

Figura 2.9: Potencia eléctrica eólica proyectada para 2020. 


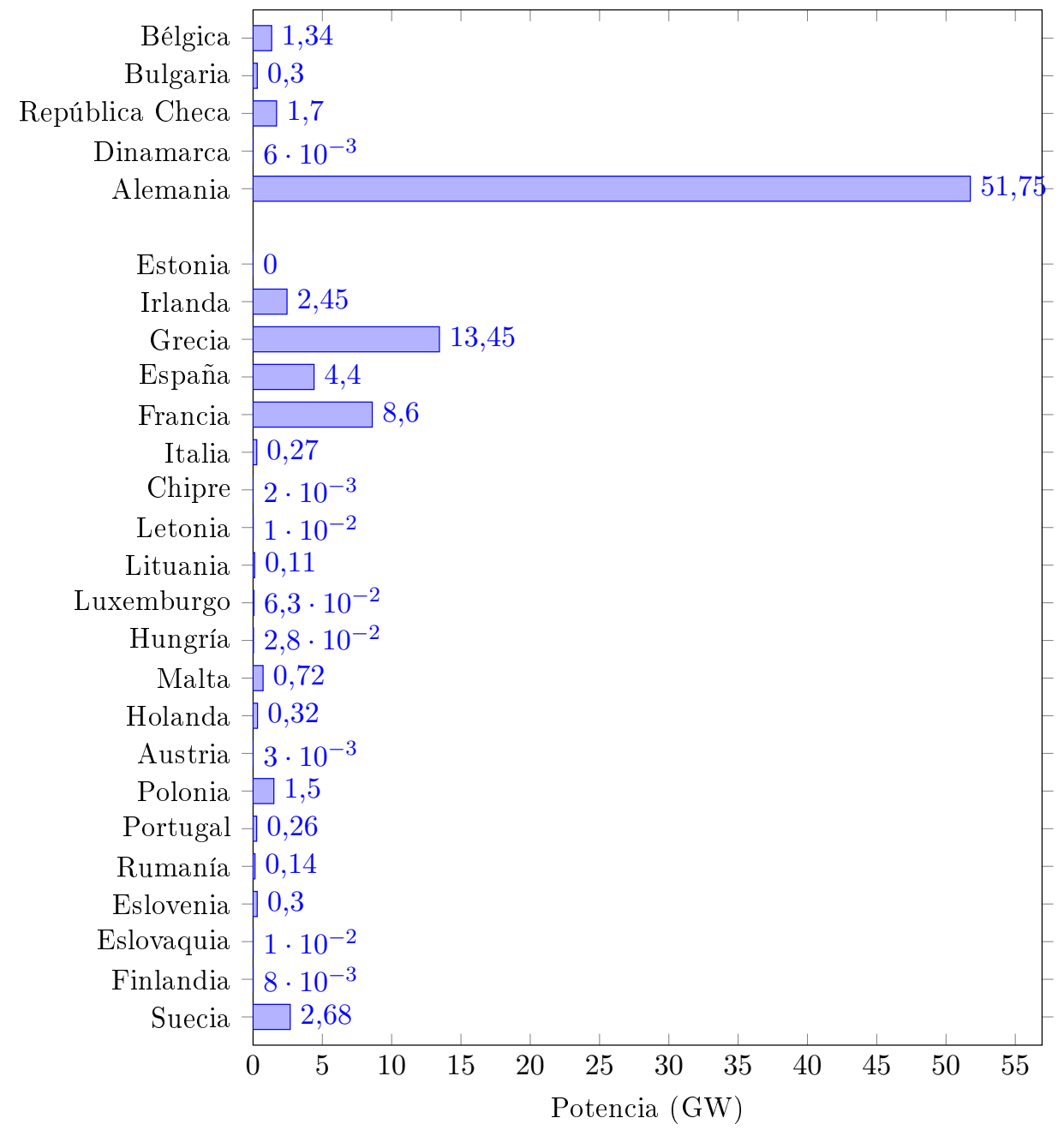

Figura 2.10: Potencia eléctrica solar proyectada para 2020. 
mo total de la energía de la UE en 2020. La Directiva 2009/28/CE asumió los objetivos anteriores y requirió a los Estados miembros la redacción de Planes de Acción Nacional de Energías Renovables (PANER). Cada Estado tienen distintos objetivos individuales para alcanzar este objetivo general, como se muestra en las figuras 2.9 y 2.10 . Además, tienen que proporcionar detalladamente las vías que describan cómo van a llegar a su objetivo [59]. Esta situación hace que se fomente parcialmente el continuo incremento de energía hidráulica así como la rápida expansión de energía eólica y FV, consideradas en este momento como FER indispensables en el mix de energía global. El rápido incremento en la integración de FER está respaldado por la caída de los precios de la tecnología empleada, en alza con los precios de los combustibles fósiles y carbón, pero su auge se debe sustancialmente a las continuas subvenciones, que pasan de 88000 millones de dolares estadounidenses (USD) en 2011 a cerca de 240000 millones USD en 2035. En ese año, 2035, las FER representarían casi un tercio de la producción total de electricidad, convirtiéndose en la segunda mayor fuente mundial de generación de energía para el año 2015 (aproximadamente la mitad que el de carbón) y, acercándose al carbón como la fuente primaria de la electricidad mundial. No obstante, las medidas de subvención para apoyar nuevos proyectos de energía renovable deben ajustarse con el tiempo a medida que aumenta la capacidad y que los costos de las tecnologías renovables caen, en un intento por evitar cargas excesivas a los gobiernos y los consumidores [38].

\subsection{Requerimientos de Conexión y Comportamien- to ante Perturbaciones.}

Antes de la última década, y debido a que el nivel de penetración de FER en la red era extremadamente bajo comparado con los sistemas convencionales de generación, no había normativa que incluyera ninguna regulación de comportamiento exigido a FER. Sin embargo, como se ha visto en el apartado anterior, esta situación ha cambiado radicalmente en muchos países Europeos, con un notable aumento de EERR integradas a la red. Debido a la naturaleza estocástica de las FER, su integración ha añadido más incertidumbre a la red, lo que conlleva a un aumento de la problemática relacionada con la disponibilidad de la generación y el control de flujo de potencia [60]. Además, la desregularización (privatización y liberalización) del sector eléctrico ha invitado a más entidades generadoras y suministradoras a satisfacer la siempre creciente demanda de electricidad [39]. Esta incorporación de las nuevas tecnologías de generación junto con un gran número de competidores ha llevado a perseguir nuevos retos para la correcta planificación y operación del presente y futuro del sistema eléctrico [61]:

- Aumento del nivel de complejidad, 
- naturaleza estocástica de la capacidad de transferencia de energía, y

- flujo de potencia bidireccional en todo el sistema.

La inclusión de estas FER junto con los estrictos requerimientos eléctricos, en términos de frecuencia y tensión que algunas cargas presentan, conlleva la aparición de problemas vinculados con la seguridad, fiabilidad y estabilidad del sistema en su conjunto, tanto desde el punto de vista de la generación como de las cargas conectadas al mismo. Así pues, algunos autores sostienen que resulta necesario un nuevo análisis que englobe el dimensionamiento adecuado de los sistemas de transporte y distribución, un estudio global de la estabilidad de los sistemas, y nuevas propuesta de conexión y operación [62. Este escenario se ve también influenciado por las características de intermitencia y fluctuación asociadas a las fuentes renovables, aspecto que ha de tenerse en cuenta para evitar desequilibrios entre generación y demanda que conlleven oscilaciones no deseadas de la frecuencia del sistema [63.

Para proteger la red eléctrica se necesita elaborar unos reglamentos y normas. Estos reglamentos y normativas actúan como procedimientos estándar y los requisitos para la inclusión o prohibición de la conexión de plantas de generación y cargas a la red. Se deben aplicar tanto a las plantas de generación y a los usuarios existentes como a las nuevos que se quieran conectar a la red. Los requerimientos de conexión son documentos técnicos que contienen las reglas y normas que rigen la operación, mantenimiento y desarrollo del sistema en los puntos de conexionado [64 dirigidos a garantizar la calidad de suministro para los consumidores [65]. A su vez, también están los requerimientos de exigencias de conexión cuando ocurre un evento, con lo cual, para minimizar los problemas de estabilidad y evitar otros inconvenientes, los operadores del sistema de eléctrico han promovido estrictos requerimientos técnicos para las instalaciones de FER, principalmente enfocadas a instalaciones eólicas [66]. Sin embargo, sólo muy pocos países (Alemania, España e Italia) tienen requerimientos técnicos específicos para plantas FV y su rendimiento bajo perturbaciones. Las normativas han ido surgiendo conforme las FER se han ido incorporando al sistema eléctrico. Por eso, la normativa empieza con aplicación a los parques eólicos y luego se extiende a FV, según va aumentando paulatinamente la cuota de demanda que estas fuentes cubren. La normativa especifica que las instalaciones bajo régimen especial tienen que soportar huecos de tensión en el punto de conexionado con el sistema de transmisión/distribución sin desconectarse de la red. Los requisitos varían de un país a otro, así como su severidad, por lo general depende del nivel de penetración de la energía eólica, así como de la robustez de la red eléctrica nacional o regional, [1], como se muestra en la figura 3.18

Como ya se ha comentado anteriormente, y en paralelo a la integración progresiva del recurso FV, algunos países han extendido los requerimientos 


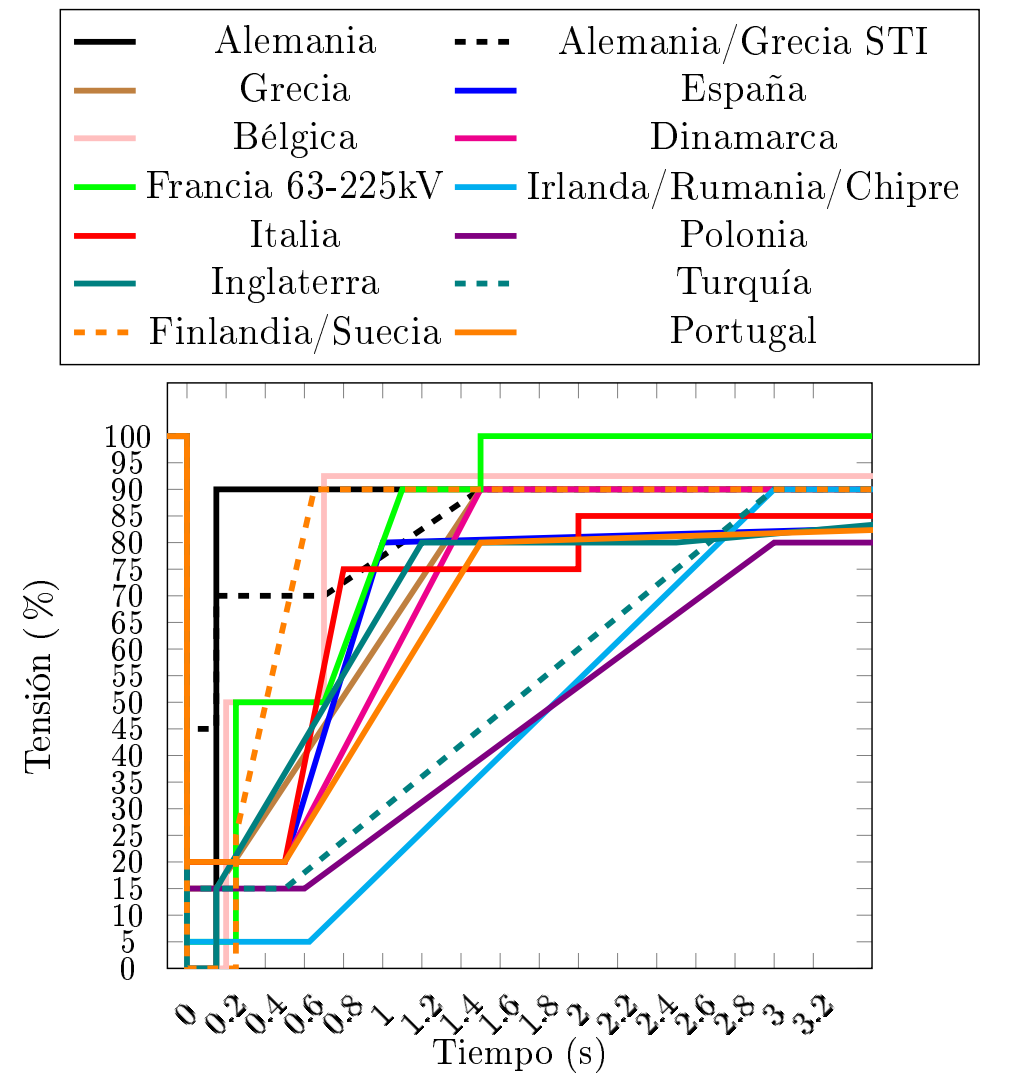

Figura 2.11: Requerimientos de conexionado para parques eólicos: Comparativa de las curvas del valor límite de tensión eficaz en el punto de conexión a la red [1]. 
de conexión ante perturbaciones del ámbito eólico al FV. Estos países son aquéllos en los que durante la última década mayor capacidad de generación FV han incorporado. A modo de ejemplo, se han considerado algunos países con una integración FV significativa, los cuales son buenos ejemplos para discutir cómo la normativa empieza a implementarse a nivel eólico y luego se extiendo a nivel FV. En el caso español también se va a hacer una pequeña retrospectiva histórica en cuanto a normativas y marcos legales.

Alemania es uno de los países con más alta penetración de energía FV de Europa, por eso también se ha visto con la necesidad de elaborar requerimientos técnicos de desconexión y comportamiento ante perturbaciones para este tipo de generadores. Al igual que la mayoría de los países europeos, los primeros requisitos de conexionado fueron específicamente para conectar los parques eólicos, se publicaron en 2003, 67]. Sin embargo, debido a la experiencia adquirida durante los últimos años y considerando nuevos avances, era necesario actualizar y modificar los requerimientos iniciales [63]. La nueva propuesta fue desarrollada por bdew (energie. Wasser. Leben) y aprobada en 2008 (Guía Técnica para Plantas de Generación Conectadas a la Red de Media Tensión) [68]. En este documento se distinguen dos tipos de plantas en función de su comportamiento bajo perturbaciones: tipo-1, donde el generador síncrono está conectado directamente (sólo a través del transformador) a la red; y tipo-2, donde se incluyen el resto de plantas de generación. Las plantas de generación secundaría tipo-1, deben mantenerse conectadas a la red si el valor eficaz de tensión se mantiene por encima de la línea roja, ver figura 2.12, evitando cualquier desconexión bajo la presencia de dichas perturbaciones. Para las plantas tipo-2, cuando se produzcan desajustes en el valor eficaz de tensión por encima de la línea punteada con etiqueta Borde de línea 1, como se puede ver en la figura 2.13, no se permite que se desconecten de la red. Actualmente, las plantas de generación no deben desconectarse de la red cuando aparezcan huecos de tensión con un $0 \%$ de valor eficaz de tensión durante una duración global de $\leq 150 \mathrm{~ms}$. Si dicho valor eficaz está entre Borde de línea 1 y la línea roja etiquetada como Borde de línea 2, las plantas de generación tienen que soportar la perturbación sin desconectarse de la red. En este área de operación se pueden dar las siguientes opciones, basadas en acuerdos con el operador del sistema [69]:

- bajo una intensidad de cortacircuito

- dependiendo del concepto de conexionado a la red, el Borde de línea 2 puede desplazarse

- desconexiones de corta duración de hasta 2s.

Por debajo de la línea azul no hay ningún requisito adicional para el lado de suministro que obligue a las plantas de generación a permanecer conectadas a la red. 


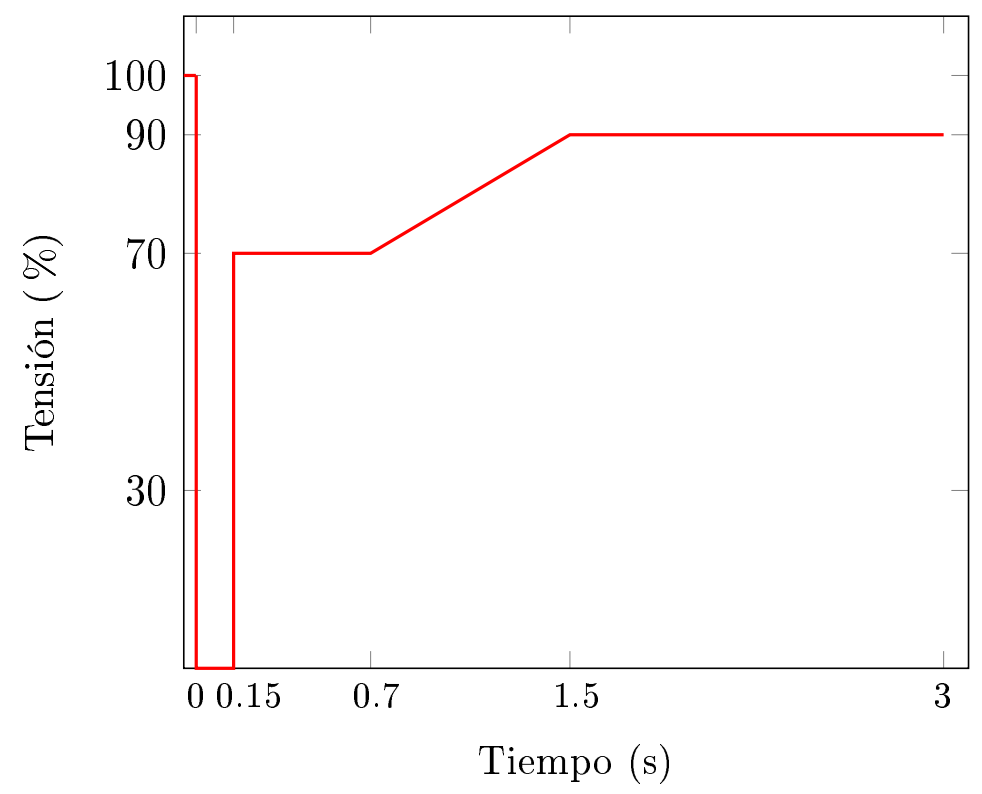

Figura 2.12: Requerimientos de conexión alemán: Curva límite de tensión para las plantas de generación tipo-1 en el punto de conexión a la red.

Todos los requerimientos recogidos en esta directiva debían estar implantados con fecha de 1 de Enero de 2009, prorrogándose hasta 2010 para el caso de instalaciones FV. Las únicas excepciones a estos requerimientos se basan en el comportamiento de las instalaciones ante procesos transitorios ocurridos en la red (por ejemplo la disminución de tensión como consecuencia de huecos) que entraron en vigor el 1 de Enero de 2010. De modo similar a la implantación de esta directiva, se otorgó a las plantas FV un año adicional (hasta el 1 de Enero de 2011) para la inclusión del comportamiento de estas ante huecos de tensión.

Durante las últimas décadas, el gobierno español ha promovido marcos legales con requisitos técnicos que regulan la conexión de generación a la red eléctrica. En España, haciendo una retrospectiva histórica, debe en primer lugar mencionarse el concepto de Generación en Régimen Especial, como definitorio de este modelo propuesto y diferenciado de los sistemas tradicionales de generación e incluidos bajo el concepto de Régimen Ordinario [70]. Nace así la Ley 54/97 y el Real Decreto (RD) 1955/2000, con el fin de definir este marco legal. Más tarde, en 2004, el gobierno español publicó el RD 436/2004, que se centró principalmente en las FER [71]. En él se obligaba a los parques eólicos a cumplir con los requerimientos y equipamiento necesarios para contribuir a la continuidad de suministro frente a perturbaciones debidas a huecos de tensión. En lo que se refiere a requerimientos de protecciones de las distintas tecnologías de generación frente a estas perturbaciones, el operador del sistema español (REE), como gestor de la red nacional, desarrolló el 


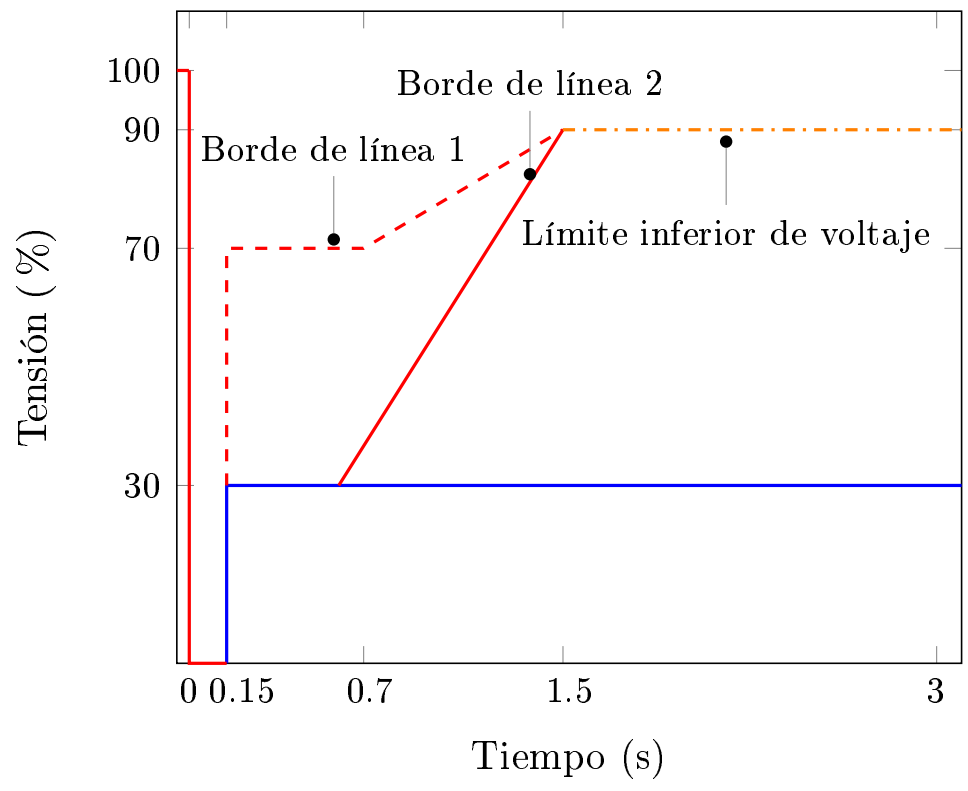

Figura 2.13: Requerimientos de conexión alemán: Curva límite de tensión para las plantas de generación tipo-2 en el punto de conexión a la red.

Procedimiento de Operación Requisitos de respuesta frente a huecos de tensión de las instalaciones de producción de régimen especial (P.O.12.3) [72]. Estos requisitos fueron aprobados y publicados en octubre de 2006, dando un carácter de obligatoriedad para parques eólicos. Posteriormente, en noviembre de 2010, se propuso y publicó el RD 1565/2010 [73], suponiendo una extensión hacia las plantas $\mathrm{FV}$ de los requisitos de continuidad en respuesta ante huecos de tensión anteriores. Adicionalmente, los requerimientos y modificaciones en el factor de potencia tienen que cumplirse para estás fuentes de energía, incluida también en el grupo de los llamados Generación en Regimen Especial (GRE). Esta extensión hacia las instalaciones basadas en solar FV vino promovida principalmente por el crecimiento tan relevante que experimentó la energía solar FV instalada en España en esos años, en los que el caso español lideró el grupo de países de mayor capacidad instalada. Específicamente, el cumplimiento del código de red en el caso español se detalla en el Procedimiento de verificación, validación y certificación de los requisitos del PO 12.3 sobre la respuesta de las instalaciones eólicas y fotovoltaicas ante huecos de tensión [4].

El marco normativo que engloba el apoyo a las fuentes renovables sufrió un importante cambio en 2013 con la publicación de la Ley 24/2013 de 26 de diciembre [74]. Entre otros aspectos, y dentro de un objetivo global de mantener un nivel de competencia efectiva en el sector eléctrico, se eliminó la diferenciación entre régimen ordinario y régimen especial, conduciéndose a una regulación unificada del sector de la generación eléctrica. Esta Ley viene 


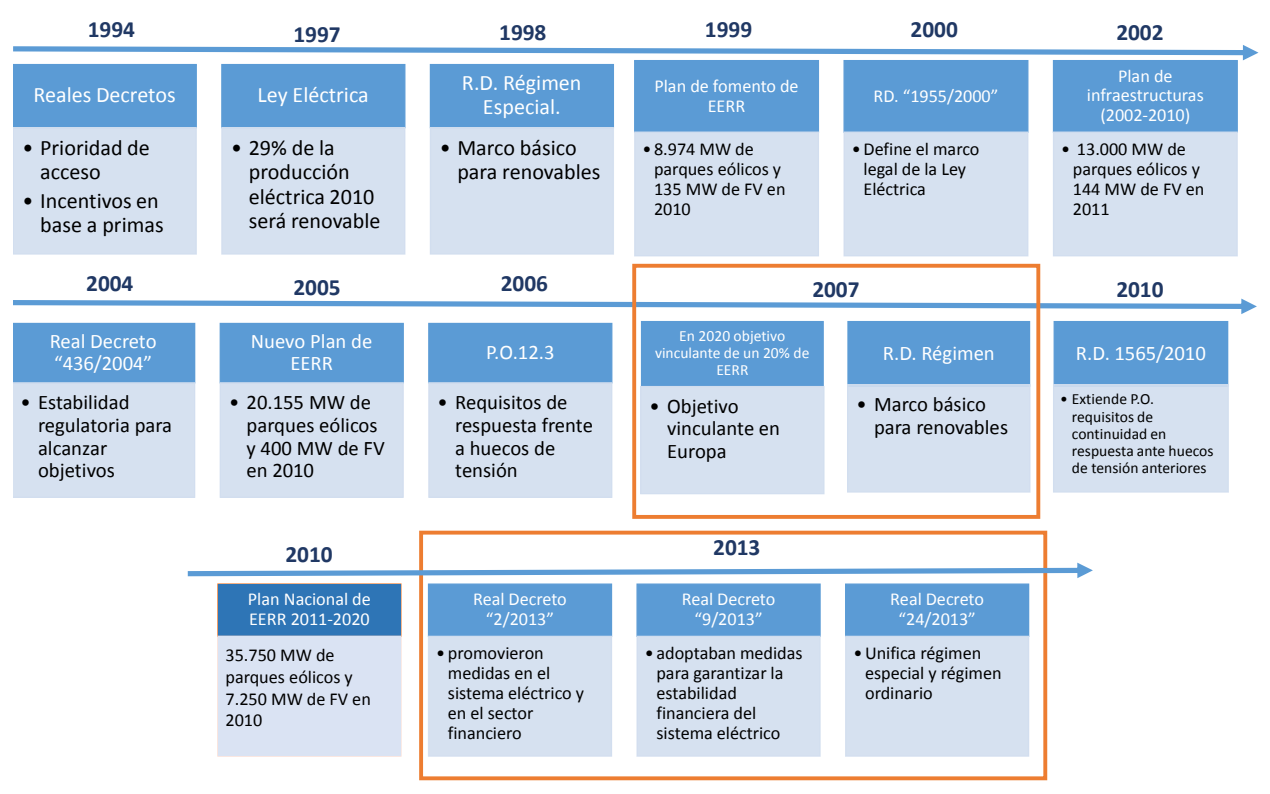

Figura 2.14: Evolución de la normativa en España

a sumarse a otras publicadas a lo largo de 2013 de especial relevancia para el sector renovable en particular, como son el Real Decreto-ley 9/2013, de 12 de julio de 2013, por el que se adoptaban medidas urgentes para garantizar la estabilidad financiera del sistema eléctrico; y el Real Decreto-ley 2/2013, de 1 de febrero de 2013 por el que se promovieron medidas urgentes en el sistema eléctrico y en el sector financiero. Con todo ello, la aparición paulatina de estas normas ha influenciado notablemente en la integración de recursos renovables en el sistema eléctrico español. De hecho, cualquier análisis económico que se realizase debería tener en cuenta este cambio de escenario y las consecuencias derivadas del mismo que, a buen seguro, influirían notablemente en las conclusiones finales.

El procedimiento español de operación para generación basada en fuentes renovables especifica que toda instalación acogida al Régimen Especial debe soportar huecos de tensión en el punto de interconexión con la red de transporte/distribución, sin desconectarse durante y después de la perturbación. Estos requerimientos se establecen para perturbaciones cuya disminución del valor eficaz de tensión (RMS) de la fase más afectada por el hueco esté dentro de los límites establecidos según recoge la figura 3.17. Los valores de tensión, de acuerdo con la normativa, han sido representados en valores por unidad de su valor nominal eficaz. Estos límites han sido establecidos en virtud a simulaciones de estabilidad del operador del sistema (REE) y a criterios asociados a los dispositivos de protección [75].

- El mínimo 0,2 pu proviene de simulaciones de estabilidad de REE y 


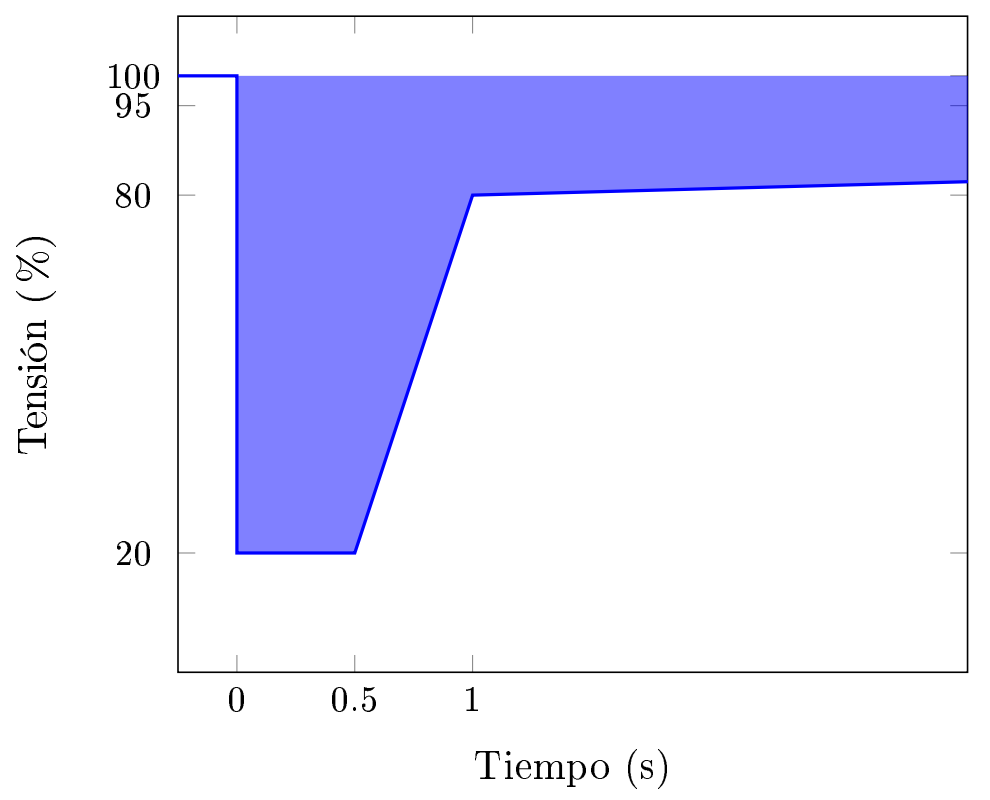

Figura 2.15: Curva española de tensión-tiempo que define el área del hueco de tensión en el punto de conexión a la red que debe ser soportado por la instalación.

el máximo genera potencia activa que la red eléctrica española puede perder cuando hay un corto circuito en el sistema de transmisión.

- El intervalo de tiempo de $500 \mathrm{~ms}$ de la caída de tensión se deriva del tiempo máximo de activación de la protección de distancia, siguiendo los criterios de protección generales del sistema eléctrico español.

- El tiempo de recuperación de la caída de tensión de hasta 1s es el resultado de la protección de bajo voltaje de las unidades de generación convencional, que se activa cuando la tensión está por debajo de $0,8 \mathrm{pu}$ durante un intervalo de tiempo mayor que 1 segundo.

Este procedimiento de operación proporciona igualmente características relacionadas con las capacidades de potencia reactiva y activa a aportar por estas instalaciones a lo largo de la perturbación. Así, durante la falta y después de ésta, incluido el período de recuperación del sistema, se debe proporcionar la máxima generación de corriente. En cualquier caso, esta corriente debe estar ubicada dentro de los límites de las curvas representadas en la figura 2.16. Tanto parques eólicos como plantas FV deben suministrar intensidad reactiva con niveles de tensión por debajo de $0,85 \mathrm{pu}$, y no deben demandar potencia reactiva entre $0,85 \mathrm{pu}$ y el nivel de tensión mínimo permitido para el normal funcionamiento de la red. Bajo estas premisas, puede entonces afirmarse que este tipo de instalaciones renovables deben mantener 


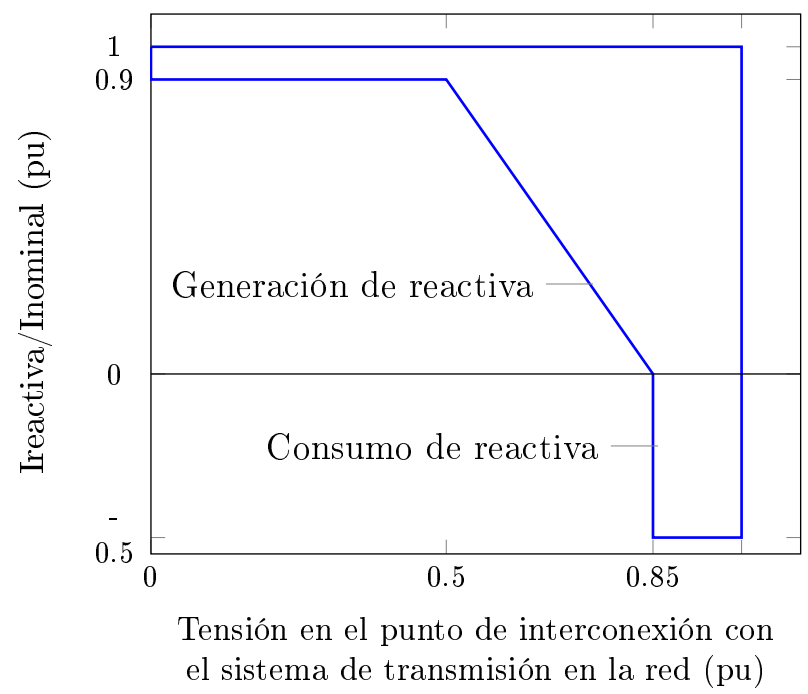

Figura 2.16: Corriente reactiva admisible (generada o consumida), en función de la tensión en bornes.

un nivel de generación de potencia activa muy similar a las condiciones de operación anteriores a la aparición de la perturbación eléctrica.

Las normas generales para los requisitos de conexión a la red de transporte en Italia se resumen en [76],[77],[78],[79]. Las principales normas técnicas para la conexión a la red de generadores eléctricos a la red italiana se encuentran en la normativa CEI 0-16(Normas técnicas de referencia para la conexión de consumidores activos y pasivos a las empresas de disatribución de red eléctrica de alta tensión (AT) y media tensión (MT)), CEI 11-20 (Sistema de producción de energía eléctrica y sistemas de alimentación ininterrumpida conectados a las redes de baja tensión (BT) y de MT), and CEI 11-32 (Sistema de producción de energía eléctrica conectada a la red de AT) [80]. Los requisitos técnicos para la conexión a la red de baja tensión para las plantas FV, cuya potencia sea superior a $6 \mathrm{~kW}$, están recogidos en la norma italiana CEI 0-21 Normas técnicas de referencia para la conexión de los usuarios activos y pasivos de las compañias eléctricas de baja tensión, Junio de 2006, como se muestra en la figura 2.17.

La normativa CEI 82-25 (Guía para el diseño e instalación de sistemas $F V$ conectados a la red de $M T$ y BT), proporciona criterios de diseño, instalación y de verificación de sistemas FV conectados a la red de BT y MT, de acuerdo con las normativas CEI 11-20 y CEI 0-16, también proporciona las prescripciones de las interfaces y la protección de dispositivos [80]. 


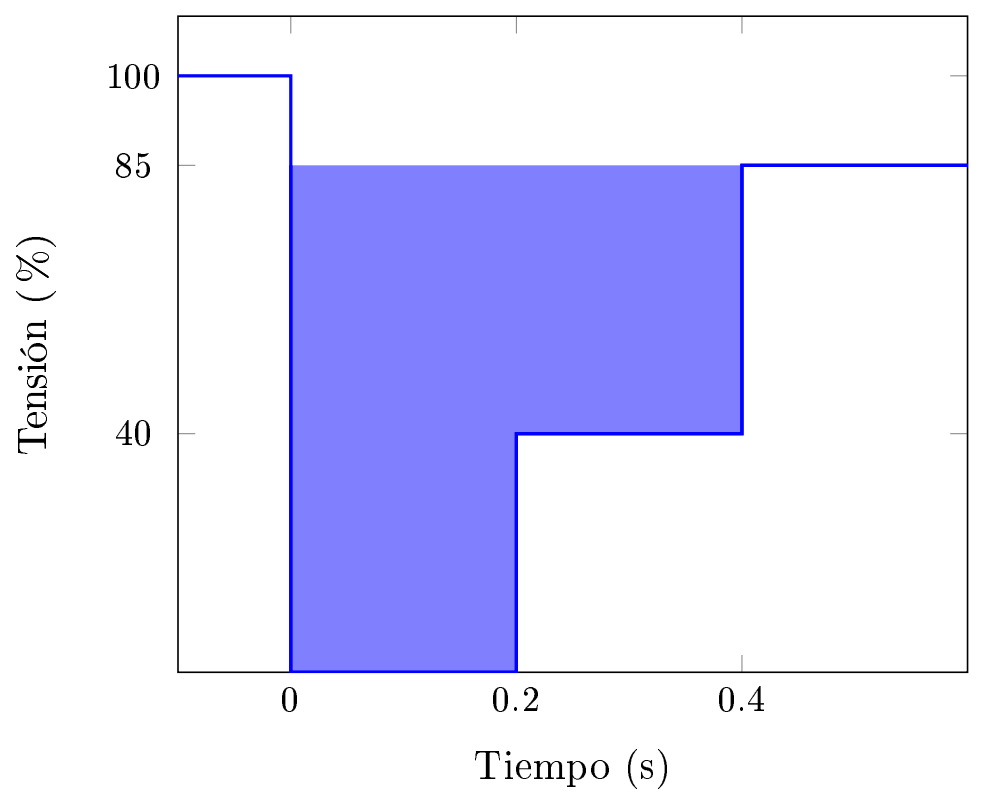

Figura 2.17: Curva italiana de tensión-tiempo que define el área del hueco de tensión en el punto de conexión a la red que debe ser soportado por la instalación.

\subsection{Propuestas en Estudio de Comportamiento an- te Perturbaciones.}

\subsubsection{Propuesta Española.}

Debido a la creciente presencia de parques eólicos e instalaciones FV en el sistema eléctrico español y, por tanto, a la cuota de demanda actualmente cubierta por recursos renovables, el operador del sistema (REE) ha planteado modificaciones de los requisitos técnicos vigentes. Este planteamiento de nuevas propuestas se inicia en 2008, publicando un borrador del P.O.12.2 con requerimientos exigibles a instalaciones eólicas, FV y todas aquellas instalaciones de producción cuya tecnología no emplee un generador síncrono conectado directamente a la red bajo el título Instalaciones conectadas a la Red de Transporte y equipo generador: requisitos minimos de diseño, equipamiento, funcionamiento, puesta en servicio y seguridad [81]. La principal diferencia de estas propuestas en comparación con las características actualmente vigentes se centra en el perfil de la curva tensión-tiempo. Así, se propone una curva característica alternativa que limita la magnitud y duración de los huecos de tensión en función del tipo de cortocircuito que produciría el hueco, englobando perturbaciones procedentes de cortocircuitos trifásicos, bifásicos a tierra o monofásicos de dos fases a tierra, tal y como se muestra 


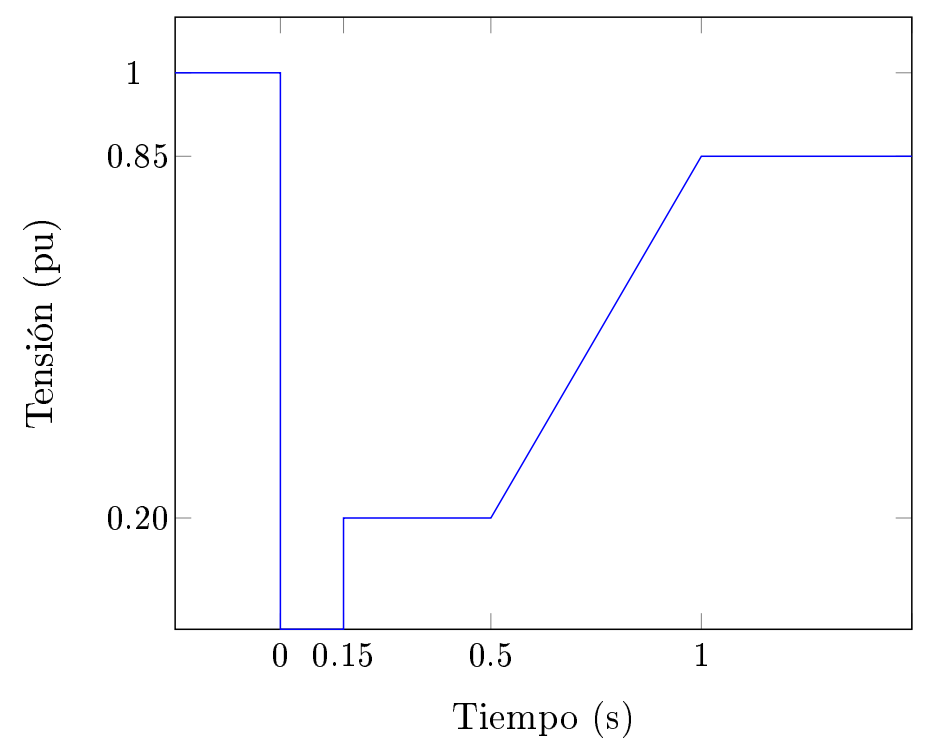

Figura 2.18: Curva propuesta de tensión-tiempo que define el área de la perturbación de tensión en el punto de conexión a red. Exceptuando el caso de cortocircuitos bifásicos aislados de tierra.

en la figura 2.18. En el caso particular de cortocircuitos bifásicos aislados de tierra, la propuesta ofrece una curva tensión-tiempo para caracterizar la magnitud y duración de este tipo de huecos de tensión que las instalaciones de generación renovable deben soportar sin desconectarse de la red, como se puede ver en la figura 2.19 .

Una característica común a requisitos actuales y propuestas en estudio es que no haya demanda de potencia activa y reactiva en el punto de conexión a la red durante la falta y a lo largo del periodo de recuperación de la misma. No obstante, y de acuerdo con el último borrador, se permitiría una demanda momentánea de potencia activa y reactiva durante los primeros instantes de aparición de la perturbación (40 ms para faltas equilibradas y de $80 \mathrm{~ms}$ para el caso de faltas desequilibradas) así como durante los 80 ms inmediatamente posteriores al despeje de la misma. En cualquier caso, y durante todo el régimen transitorio en que está presente la perturbación incluyendo el intervalo de recuperación de la misma, la instalación debe ser capaz de inyectar a la red una corriente aparente de, al menos, el valor de la intensidad nominal de la instalación. Finalmente, la figura 2.20 compara los perfiles característicos de tensión-tiempo del último borrador P.O.12.2 publicado frente a los requisitos de conexión actualmente vigentes en España. 


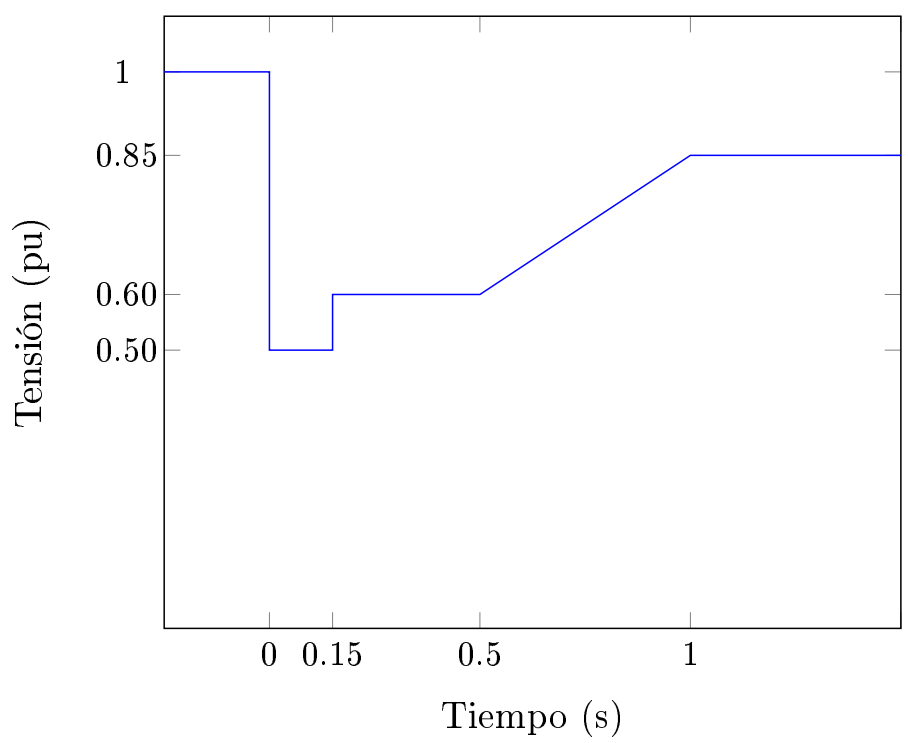

Figura 2.19: Curva propuesta de tensión-tiempo que define el área de la perturbación de tensión en el punto de conexión a red. Caso de cortocircuitos bifásicos aislados de tierra.

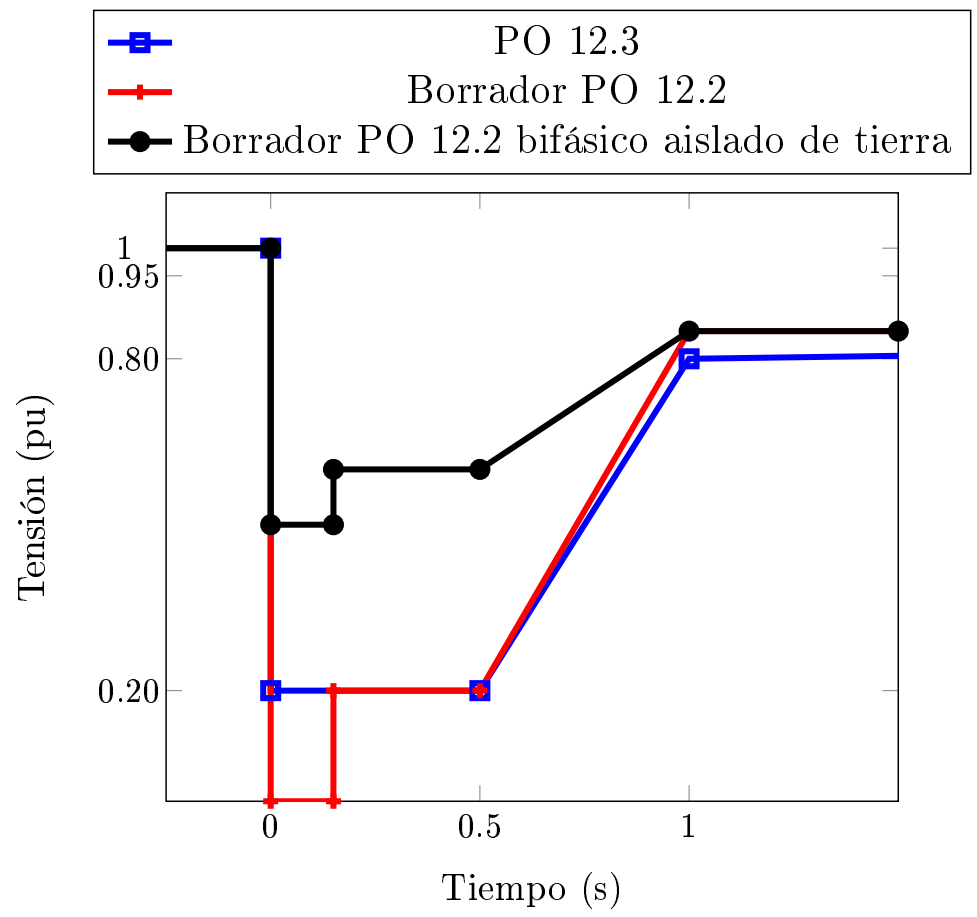

Figura 2.20: Comparativa de requerimientos tensión-tiempo. Normativa actual y propuesta en estudio. 


\subsubsection{Propuesta de ENTSO-E.}

Según se ha visto en las secciones anteriores, no hay un único requerimiento para los países europeos. Estos varían de un país a otro, más aún no todos los países europeos han desarrollado normas específicas para FER conectadas a la red. A nivel europeo se han promovido diferentes iniciativas y requerimientos para fuentes renovables ante la presencia de perturbaciones. Esta proliferación de diferentes criterios se está intentado homogeneizar desde el grupo de operadores de sistemas eléctricos europeos (ENTSO-E), con el fin de establecer pautas de comportamiento comunes en los diferentes países. De hecho, en 2009 ya se apuntaba esta diversidad de requerimientos como un importante hándicap para la integración de recursos renovables, [82] 83, enumerándose una serie de proyectos e iniciativas europeas encaminadas a promover normativas comunes. Igualmente, se recuerda que estos requerimientos han de estar acorde con los estándares europeos que incluyen limitaciones generales para la conexión de suministro a redes eléctricas. En concreto, para la conexión a redes de baja tensión ha de tenerse en cuenta la norma europea EN 50438. En lo que se refiere a especificaciones de calidad de suministro, mientras que para los consumidores finales se establece la norma EN 50160, no existe sin embargo una normativa de calado internacional que hayan de cumplir los generadores renovables.

Bajo este escenario, y como una forma de proporcionar normas generales para las nuevas instalaciones de generación conectadas a la red, se presenta el ultimo borrador de código de red sobre requerimientos de plantas de generación eléctrica (impulsado por ENTSO-E) que proporciona guías generales para la implementación a nivel nacional de puntos de generación conectados por debajo de $110 \mathrm{kV}$ dentro de países de la Unión Europea. Estos requerimientos están enfocados a evitar desconexiones indeseadas de puntos de generación renovable conectados a red, ante la presencia de perturbaciones producidas en el sistema de transporte por faltas (cortocircuitos) correctamente despejadas. El objetivo es limitar las pérdidas de generación durante y después de una falta en el sistema de distribución o transmisión para niveles de tensión de $110 \mathrm{kV}$ o mayores. La figura 2.21 muestra los límites del valor eficaz de tensión durante la falta y después de su despeje. Las plantas de generación deberán estar conectadas a la red y continuar con la operación estable para tensiones superiores a estos límites [84]. 


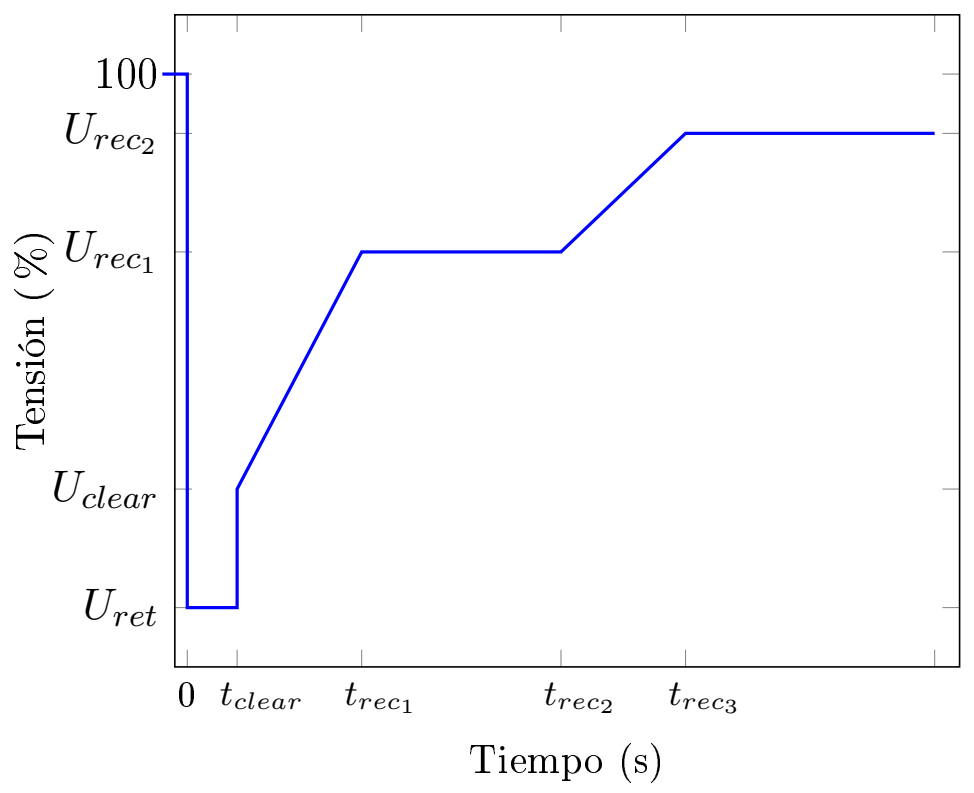

Figura 2.21: Guía para los requerimientos de conexión: Curva límite de tensión para las plantas de generación en el punto de conexión a la red.

\begin{tabular}{|c|c|c|c|c|c|c|c|}
\hline \multicolumn{4}{|c|}{ Generadores síncronos } & \multicolumn{4}{|c|}{ Potencia de parques modulares } \\
\hline Parám & tros de voltaje $(\mathrm{pu})$ & Parán & s de tiempo (s) & Parám & de tensión (pu) & Pará & $\mathrm{s}$ de tiempo $(\mathrm{s})$ \\
\hline$U_{\text {ret }}$ & $0.05-0.3$ & $t_{\text {clear }}$ & $0.14-0.25$ & $U_{\text {ret }}$ & $0.05-0.15$ & $t_{\text {clear }}$ & $0.14-0.25$ \\
\hline$U_{\text {clear }}$ & $0.7-0.9$ & $t_{r e c_{1}}$ & $t_{\text {clear }}$ & $U_{\text {clear }}$ & $U_{\text {ret }}-0.15$ & $t_{r e c_{1}}$ & $t_{\text {clear }}$ \\
\hline$U_{r e c_{1}}$ & $U_{\text {clear }}$ & $t_{r e c_{2}}$ & $t_{r e c_{1}}-0.7$ & $U_{r e c_{1}}$ & $U_{\text {clear }}$ & $t_{r e c_{2}}$ & $t_{r e c_{1}}$ \\
\hline$U_{r e c_{2}}$ & $0.85-0.9$ y $U_{\text {clear }}$ & $t_{r e c_{3}}$ & $t_{r e c_{2}}-1.5$ & $U_{r e c_{2}}$ & 0.85 & $t_{r e c_{3}}$ & $1.5-3.0$ \\
\hline
\end{tabular}

Tabla 2.2: Guía para los requerimientos de conexión: parámetros para tiempo y tensión dentro de los rangos definidos por la normativa. 


\section{Capítulo 3}

\section{Propuesta de Caracterización de Huecos de Tensión y Requerimientos de Operación.}

\footnotetext{
Resumen: Los estudios de huecos de tensión y perturbaciones comienzan en los primeros años de la década de los 90, donde aparecen las primeras propuestas de caracterización y representación de estos eventos. De forma paralela,surgen normativas y requerimientos de conexión vinculados con este tipo de perturbaciones, la mayoría de ellos basados en establecer una trayectoria límite para el valor eficaz de la fase más afectada por la perturbación. En este escenario, el presente capítulo recoge un estado del arte de las caracterizaciones más representativas de los huecos de tensión, tanto a nivel individual como a nivel de campañas de medidas. Posteriormente, introduce un parámetro adicional para determinar con mayor detalle la severidad de los huecos, considerando el intervalo de tiempo marginal que el valor eficaz se mantiene cercano a su valor mínimo. Finalmente, propone un conjunto de longitudes como herramienta identificativa y de visualización de las trayectorias de los valores eficaces a lo largo de la perturbación. Estas longitudes recogen de manera resumida pero concisa las principales características de este tipo de eventos, y permiten una comparativa eficiente y ágil con los requisitos de los operadores de sistema.
} 


\subsection{Introducción.}

El sector eléctrico supone en la actualidad una solución de distribución y consumo de energía altamente demandada y prioritaria en nuestra sociedad contemporánea. De hecho, los consumidores cuentan cada vez más con tecnologías y equipamiento directamente ligado y dependiente del consumo eléctrico. En muchos casos, estos equipos y dispositivos se han convertido en imprescindibles para la vida diaria y el correcto funcionamiento de los procesos industriales. Como resultado de todo ello, se espera que la demanda de electricidad crezca más que cualquier otra forma final de energía. Así, el escenario previsto a medio plazo por la Agencia Internacional de la Energía (IEA World Energy Outlook) estima que la demanda de electricidad se incrementará en más de dos tercios en el período de 2011 a 2035. No obstante, no es sólo la demanda energética la que está sufriendo modificaciones, sino también el mix de generación eléctrica, sobre todo como consecuencia de la integración de fuentes renovables. En consecuencia, las previsiones actuales vaticinan que la participación del carbón caerá del $41 \%$ al $33 \%$ en 2035 , aunque seguirá siendo la fuente de generación con mayor cuota. El gas representará alrededor del $22 \%$, mientras que los combustibles fósiles supondrán más de la mitad de toda la generación. En cuanto a las energías renovables, éstas se espera que presenten un incremento del $20 \%$ al $31 \%$ [85], alentadas tanto por políticas de reducción de emisiones de gases de efecto invernadero como por iniciativas de aprovechamiento de fuentes no convencionales.

En lo que respecta a los sistemas eléctricos, su objetivo principal podría resumirse en asegurar un suministro fiable de energía eléctrica a todos los consumidores conectados a red. Esta disponibilidad energética debería estar cubierta en cualquier instante y con la capacidad necesaria para atender las horas de máxima demanda. Además de este objetivo fundamental, la energía eléctrica debe cumplir con unos requisitos de calidad que garanticen la estabilidad del nivel de tensión y la continuidad del servicio [70]. Aparece así el concepto de calidad de suministro de energía eléctrica, temática de gran actualidad y que es motivo de preocupación tanto por parte de los suministradores como por parte de los usuarios [86]. El término de Calidad de Suministro se refiere a una amplia variedad de fenómenos electromagnéticos caracterizados por la evolución particular de la tensión e intensidad en un intervalo de tiempo dado y en una zona acotada del sistema eléctrico [87]. La Calidad de Suministro puede también ser referida en la bibliografía específica como Calidad de Tensión o Calidad de Intensidad. Entre todas las perturbaciones electromagnéticas que se conocen, es de destacar desde el punto de vista del consumidor aquéllas que implican una reducción en la tensión de alimentación, debido a la cada vez mayor sensibilidad con la tensión de las cargas conectadas a red. En este sentido, las reducciones de tensión más notables están habitualmente causadas por cortocircuitos o faltas a tierra 
ocurridos cerca de subestaciones, produciendo una reducción muy acusada de la tensión en una o en más fases. Disminuciones de tensión habitualmente de menor importancia suelen deberse a transitorios asociados a grandes cargas, que provocan una alteración de la tensión de red en la zona próxima al consumo. Habitualmente, las reducciones en la tensión de una o varias fases suelen permanecer presentes en el sistema un tiempo muy reducido, debido a la rápida acción de maniobras de desconexión automáticas, reparaciones de las faltas o estabilización de las cargas. Así pues, y dependiendo de la duración y de la tensión mínima alcanzada durante el evento, este fenómeno de reducción de tensión se puede clasificar como huecos de tensión o como interrupción [88]. En [89] se tiene una clasificación más detallada, distinguiéndose entre:

- Huecos de tensión.

- Interrupciones momentáneas.

- Interrupciones prolongadas.

En cuanto a los efectos producidos por estas perturbaciones, en general se consideran los huecos de tensión menos perjudiciales para el cliente que las interrupciones, tanto momentáneas como prolongadas. Sin embargo, y dado que el número de huecos de tensión es mayor - las estadísticas indican que en los países desarrollados el ratio de huecos de tensión frente a interrupciones es de 10:1 [90] - su repercusión total puede considerarse de mayor entidad que el producido globalmente por las interrupciones [91, 92]. No obstante, y dado que los huecos de tensión poseen una duración bastante menor que las interrupciones, su registro y análisis resulta más complejo en comparación con las tradicionales interrupciones de larga duración [93]. Por este motivo, diversos autores abogan por líneas de seguimiento y diagnóstico de calidad de suministro cada vez más completas, con el fin de hacer frente y conocer este tipo de perturbaciones de la forma más precisa posible [94]. De hecho, existe muy poca informacion relativa a la presencia de huecos de tensión y su infuencia sobre los sistemas eléctricos con anterioridad a 1990 [95]; datándose a principios de los 90 algunos de los estudios de calidad más relevantes llevados a cabo hasta la fecha [96] [97 [5] [90]. Con la integración paulatina de las energías renovables a la generación eléctrica, los huecos de tensión se vienen considerando como uno de los temas prioritarios y de los retos de mayor actualidad [98].

Despues de una revisión precisa de la normativa vigente, puede afirmarse que no hay definición única de hueco de tensión. Como hueco de tensión quedan englobadas reducciones de la tensión en un punto del sistema eléctrico por debajo de un umbral especifico, elegido para el propósito de detectar y seguir la recuperación de la tensión después de un corto período de tiempo (duración del hueco) que va desde medio de un ciclo a unos pocos segundos 


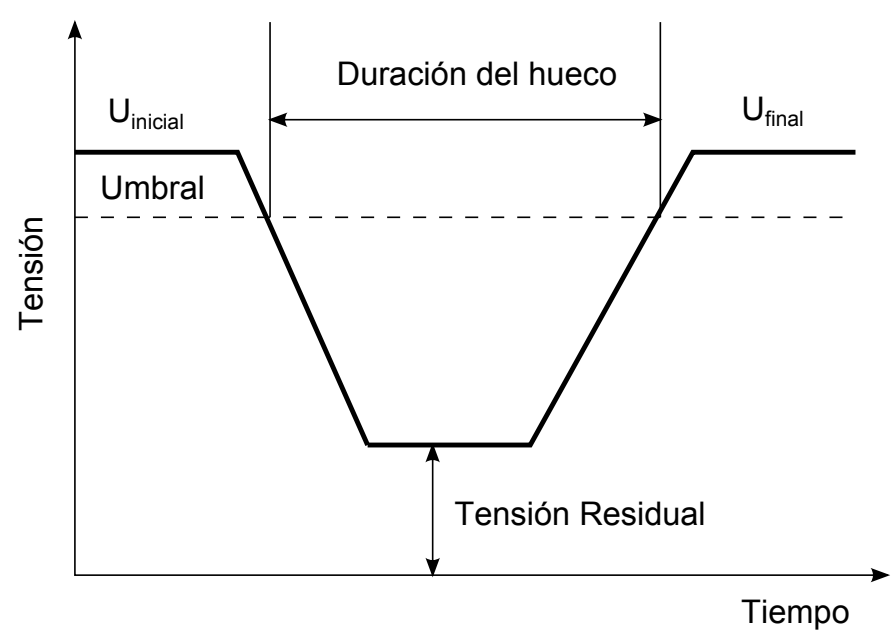

Figura 3.1: Hueco de tensión.

22. La figura 3.1 muestra una representación simplificada de un hueco de tensión y los parámetros principales. En dicha figura puede apreciarse una tensión senosoidal estacionaria incialmente de amplitud $U_{\text {inicial }}$, la cual comienza a disminuir hasta que atraviesa un valor umbral establecido como límite admisible y se inicia con ello el hueco. Posteriormente se alcanza el valor mínimo de la tensión eficaz, $U_{\text {residual }}$ y comienza la recupración de la tensión, período que abarca hasta que ésta retoma valores por encima del umbral admisible $U_{\text {final }}$. En el último IEEE Recommended Practice for Monitoring Power Quality [87] se define hueco de tensión como toda disminución en el valor eficaz de tensión que se produzca entre el $90 \%$ y el $10 \%$ del valor de la tensión nominal, abarcando una duración temporal entre medio ciclo y un minuto.

En el caso de que el hueco de tensión afecte a más de una de las fases, la mayoría de normativas y definiciones se centran y hacen referencia a la fase con menor tensión residual, contabilizando la duración del evento como el intervalo de tiempo total en que la tensión eficaz en al menos una de las fases siga siendo inferior al $90 \%$, tal y como se indica en la Normativa IEC 61000-4-30 [99]. Como puede deducirse, una desventaja importante que plantea este método de caracterización es que un hueco de tensión en una sola fase se vería reprentado en términos de tensión residual y tiempo de igual manera igual que un hueco trifásico de la misma profundidad. Sin embargo, los huecos trifásicos son más severos para las cargas que los huecos asimétricos que tuviesen similar duración y tensión residual [2].

La caracterización de hueco de tensión puede definirse como la cuantificación a través de un número limitado de parámetros de las alteraciones producidas en la evolución de la tensión [100]. De acuerdo con la bibliografía 
específica, los huecos de tensión han sido habitualmente caracterizados por los siguientes parámetros: duración, magnitud (en cuanto a tensión eficaz mínima alcanzada) y salto de fase (como consecuencia de la dinámica generada por la perturbación) [3]. En cuanto a la severidad de los huecos de tensión, habitualmente ésta se ha asociado a su duración global y a la disminución máxima de tensión eficaz — denominada tensión residual—. Principalmente, la severidad de las perturbaciones ha estado vinculada con dicha tensión mínima. Sin embargo, y a la luz del análisis de trayectorias reales de huecos, esta definición de severidad no tiene en cuenta la evolución propia de la tensión eficaz a lo largo del hueco, sino que sólo contempla el valor mínimo alcanzado. De este modo, se caracterizarían de igual forma eventos con la misma tensión mínima pero que presentasen trayectorias de disminución de tensión y recuperación totalmente diferentes. Así, perturbaciones que permaneciesen un alto porcentaje de tiempo en este valor mínimo serían catalogadas de similar severidad a perturbaciones en cuyo valor residual permaneciese unos pocos instantes. En este contexto, sería, pues conveniente introducir de alguna manera la trayectoria seguida por la tensión eficaz dentro de una perturbación como medida de severidad de hueco, con el fin de diferenciar severidades en función del esfuerzo requerido a los elementos conectados al sistema de eléctrico - tanto elementos del lado de la demanda como de la generación-. Sin embargo, y de acuerdo con la literatura consultada, la mayoría de las aportaciones proponen sólo los parámetros de magnitud y duración del hueco para cuantificar la severidad del hueco. Es más, el uso de estos parámetros no aporta información relevante sobre el hueco, centrando los valores en la fase más afectada por el evento y descartando el resto de fases, [94], [2], 27], [101]. Esta aproximación, apropiada para huecos equilibrados, no presenta resultados plausibles para huecos desequilibrados [27]. Por esta razón, algunos autores proponen diferentes métodos para caracterizar huecos desequilibrados, basándose en mediciones o considerando modelos eléctricos simplificados [100]. Otros autores consideran que información adicional del hueco de tensión puede ser relevante, tales como la profundidad del hueco y el ángulo de fase durante todo el evento [2], o las tensiones de fase individuales durante la perturbación [102].

A modo de resumen, pueden identificarse las siguientes metodologías para la caracterización de huecos [28]:

- El control de la tensión residual más baja y el tiempo de duración de hueco más largo de las tres fases de tensión.

- El seguimiento de todas las formas de onda de tensión con una adecuada frecuencia de muestreo.

- El seguimiento de $\sqrt{V_{d}^{2}+V_{q}^{2}}$ mediante un vector de control [103]. 


\subsection{Caracterización y Representación de Huecos de Tensión. Estado del Arte.}

\subsubsection{Caracterización mediante RMS.}

Las actuales normativas técnicas sobre conexionado de plantas eólicas y FV toman como referencia la tensión eficaz de la fase más afectada por la perturbación como una estimación de la situación más desfavorable del evento, y obligan a las instalaciones de generación a cumplir con ciertas limitaciones de tensión y tiempo para mantener la conexión a red durante la perturbación. Así pues, y bajo la premisa de que las normativas europeas establecen los perfiles admisibles de tensión eficaz mediante el método de representación de trayectorias rms, se concluye de forma natural la necesidad de evaluar la severidad y el rendimiento esperado de hueco mediante dicho método de representación. Así, puede considerarse la representación por rms como un método convencional de caracterización de eventos, donde el hueco de tensión queda determinado por su magnitud y duración [99], [104], [105]. En este sentido, en [87] se define un hueco de tensión como una reducción en la magnitud de tensión rms en un rango de tiempo. De esta manera, se entiende por duración del hueco aquel intervalo de tiempo en el que los valores de tensión eficaz se mantienen menores a un umbral específico. Tanto en situaciones de campañas reales de medida como en simulación de eventos, los valores eficaces de tensión son estimados a partir de valores instantáneos de tensión. Así, la determinación del valor eficaz se lleva a cabo atendiendo a la siguiente expresión[92],[105]:

$$
V_{r m s}=\sqrt{\frac{1}{N} \cdot \sum_{i=1}^{N} v_{i}^{2}}
$$

donde $N$ es el número de puntos medidos o simulados por ciclo y $v_{i}$ tensión instantánea medida. La forma de onda $V_{r m s}$ está retrasada en relación con la tensión de fase por $\mathrm{N}-1$ puntos de medida debido al uso de una ventana deslizante de N-muestras, usada en la ecuación 3.1 .

El resultado de la trayectoria seguida por la tensión eficaz depende de cómo se definan el número de muestras por ciclo. Los métodos convencionales calculan un valor de tensión eficaz por ciclo [94]. De este modo se obtienen pocos valores y cambios bruscos de rms ante la presencia de perturbaciones, tal y como muestran las figuras $3.2 \mathrm{c}$ y $3.3 \mathrm{c}$. Este método puede ser preferible en algunos casos, como en el cálculo de índices de funcionamiento basados en niveles de tensión rms [106]. Este método convencional asume que el perfil eficaz durante el hueco de tensión va a seguir trayectorias ciertamente alejadas de la evolución instantánea de la tensión. Esta hipótesis simplificativa puede no resultar adecuada para la estimación y caracterización de 
eventos reales, los cuales presentan perfiles muy heterogéneos, sin seguir un patrón siempre de carácter rectangular, lo que supone una clara desventaja en cuanto a precisión de este método [107], [108]. Para perturbaciones no rectangulares, y con el fin de establecer una estimación más precisa de la evolución de las tensiones, se puede generar una ventana deslizante de forma que el valor eficaz se determina con una paso de tiempo igual al intervalo entre valores medidos de tensión. De esta forma se obtiene una evolución más detallada del valor eficaz, detectando con mayor precisión el inicio de hueco, tal y como se puede ver en la figura 3.3e. Evidentemente, con evoluciones de perturbaciones más complejas, las diferencias entre ambas metodologías serían más acusadas.

Así pues, y atendiendo a todo lo comentado hasta ahora y teniendo en cuenta los resultados reflejados en las figuras anteriores, se decide estimar el valor eficaz de tensión a partir de una ventana deslizante cuyo desplazamiento es función del tiempo de muestreo de los datos, ver figura 3.4. Entre las ventajas a destacar de esta aproximación destaca el hecho de que puede establecerse dentro del propio ciclo de la onda el instante en el que se inicia la disminución del valor eficaz y por tanto el inicio del hueco de tensión. Igualmente, se puede definir con la precisión que otorgue el tiempo de muestreo el instante de despeje de la falta. Por tanto, puede afirmarse que los instantes concretos de inicio o finalización de hueco serán función de la metodología implementada para el cálculo de valores eficaces.

En función de los valores rms estimados existen diferentes clasificaciones de huecos atendiendo a la evolución propia de este parámetro. Uno de los más conocidos es la clasificación fasorial de huecos de tensión - Clasificación $A B C$ - donde inicialmente se distinguen cuatro tipos de perturbaciones, [109]. Esta clasificación fue posteriormente extendida a siete tipos incluyendo faltas bifásicas a tierra. Las expresiones para los siete tipos de tensiones están recogidos en [2]. Esta caracterización considera tanto el módulo como el ángulo de los fasores de tensión en las tres fases durante el hueco. La tabla 3.1 y figura 3.1 muestran los cambios sufridos por estos huecos de tensión ante la presencia de transformadores. Este método presenta como inconvenientes principales que sólo se basa en simulaciones y que no se puede conocer el tipo de hueco mediante la curva de tensión eficaz [2].

Otra clasificación de huecos de tensión que puede encontrarse en la bibliografía específica se basa en el empleo de componentes simétricas clasificación de la componente simétrica - [2]. En este caso, se distingue entre hueco principal que afecte a una sola fase, o bien que afecte a dos fases. Los huecos producidos por faltas trifásicas, suponiendo una misma disminución de valor eficaz en las tres fases, se considera un caso límite para huecos monofásicos y bifásicos. La tensión en la secuencia cero es una característica que se trata de forma separada. Las otras dos variables a considerar son: (i) tensión característica, $V$, y (ii) factor $P N, F$. La expresión general 

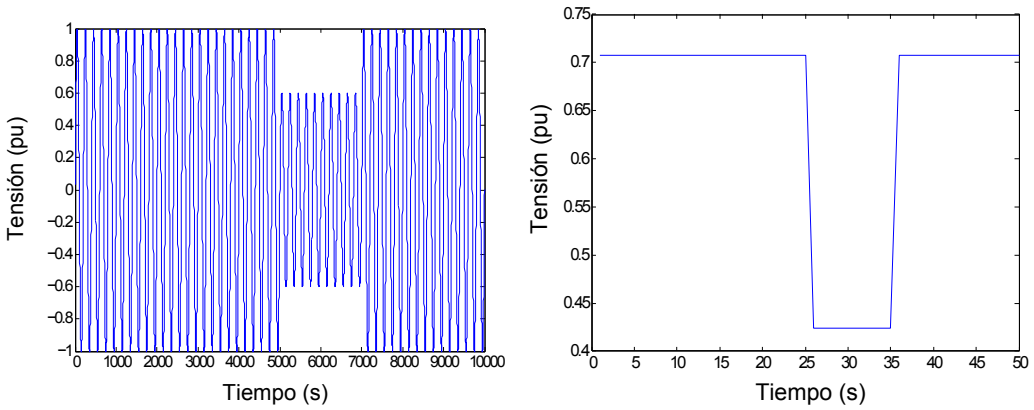

(a) Valores de tensión instatáneos

(b) Valores estimados de rms por ciclo de tensión
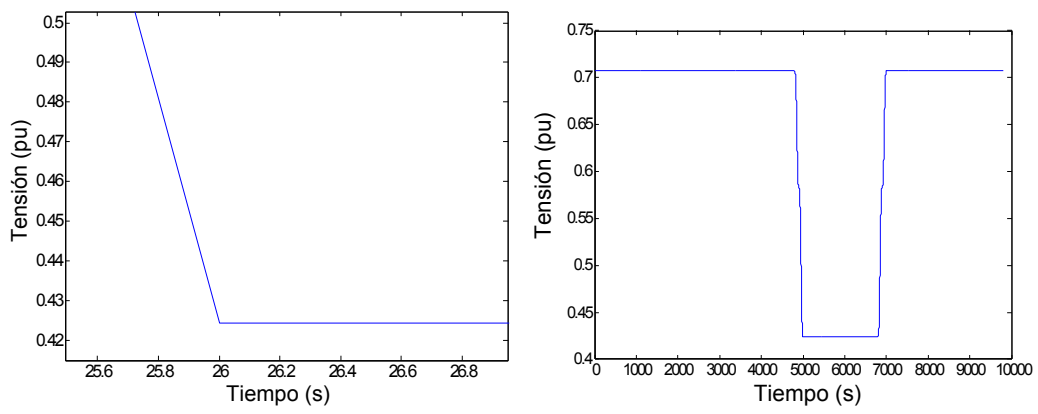

(c) Zoom de valores estimados de rms(d) Valores estimados de rms por inpor ciclo de tensión tervalo de muestreo

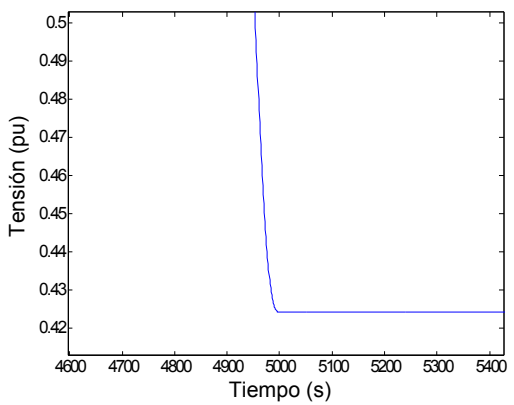

(e) Zoom valores estimados de rms por intervalo de muestreo

Figura 3.2: Ejemplo de hueco rectangular. 

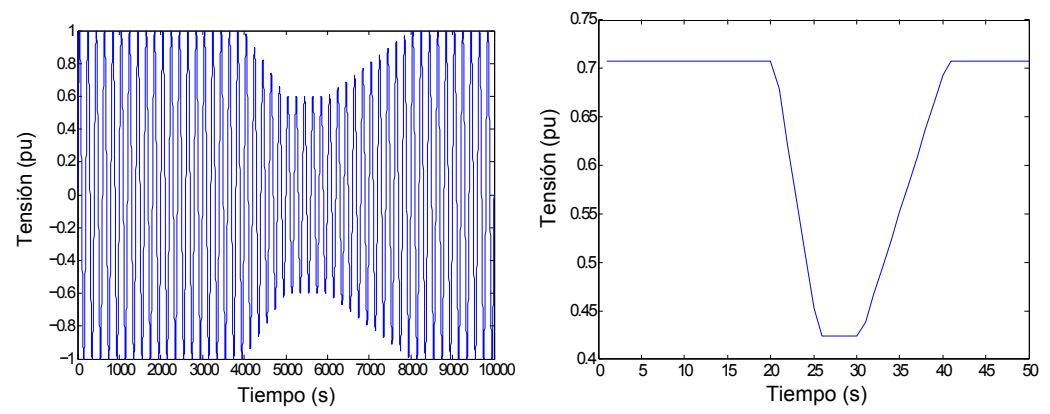

(a) Valores de tensión instatáneos

(b) Valores estimados de rms por ciclo de tensión
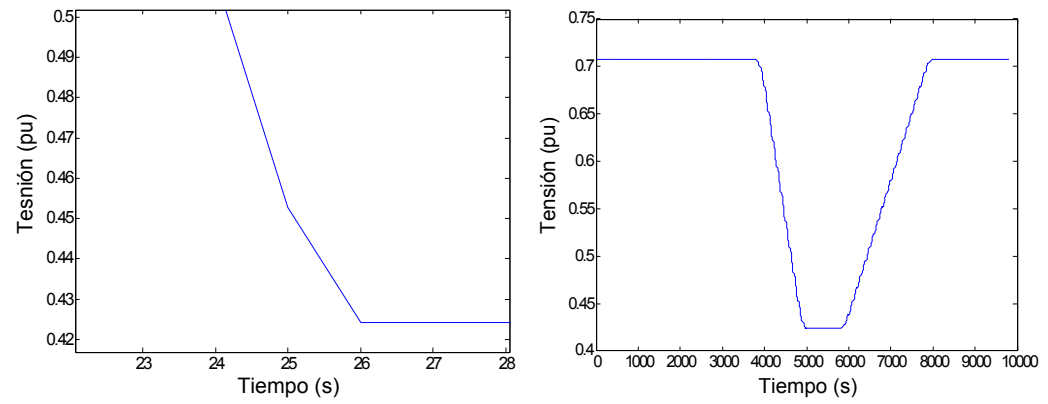

(c) Zoom valores estimados de rms(d) Valores estimados de rms por inpor ciclo de tensión tervalo de muestreo

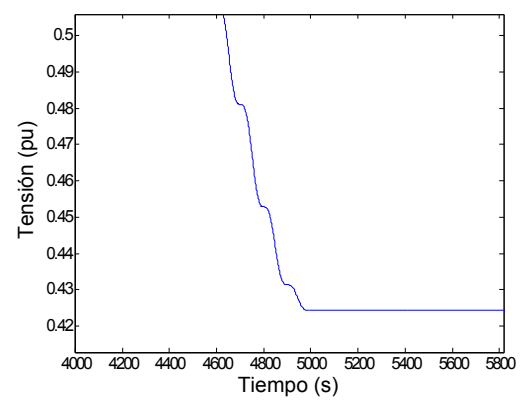

(e) Zoom valores estimados de rms por intervalo de muestreo

Figura 3.3: Ejemplo de hueco no rectangular. 


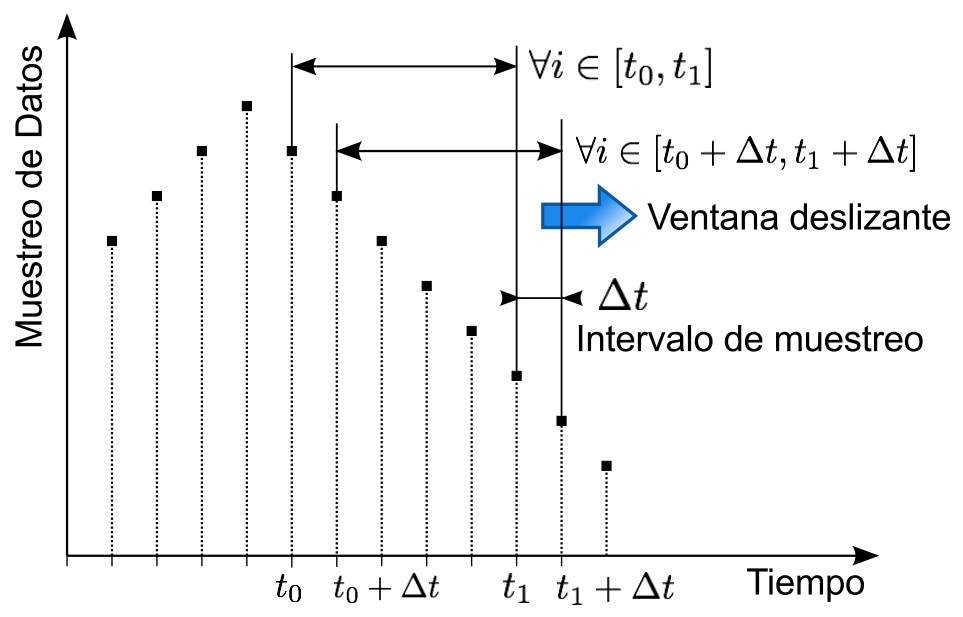

Figura 3.4: Ventana deslizante.

\begin{tabular}{l|clc}
\hline \multirow{2}{*}{ Tipo de falta } & \multicolumn{3}{|c}{ Localización de la medida } \\
\cline { 2 - 4 } & I & II & III \\
\hline Trifásico & A & A & A \\
Bifásico a tierra & E & F & G \\
Bifásico aislado & C & D & C \\
Monofásico a tierra & B & C & D \\
\hline
\end{tabular}

Tabla 3.1: Huecos a diferentes niveles de tensión debidos a diferentes tipos de faltas [2].

para la componente de secuencia no cero del hueco de tensión con el hueco principal en la fase a (tipo Da) viene dada por:

$$
\begin{array}{lc}
U_{a}= & V \\
U_{b}= & -\frac{1}{2} V-\frac{1}{2} j F \sqrt{3} \\
U_{c}= & -\frac{1}{2} V+\frac{1}{2} j F \sqrt{3} .
\end{array}
$$

La expresión general para huecos de tensión con el hueco principal entre las fases b y c (hueco tipo Ca), es:

$$
\begin{array}{lc}
U_{a}= & F \\
U_{b}= & -\frac{1}{2} F-\frac{1}{2} j V \sqrt{3} \\
U_{c}= & -\frac{1}{2} F+\frac{1}{2} j V \sqrt{3},
\end{array}
$$

donde $|F| \geq|V|$. Para huecos equilibrados debidos a faltas trifásicas, se tiene que $F=V$. Expresiones similares se pueden encontrar para los otros cuatro tipos de hueco: Db (hueco en la fase b), Dc (hueco en la fase c); Cb (hueco 


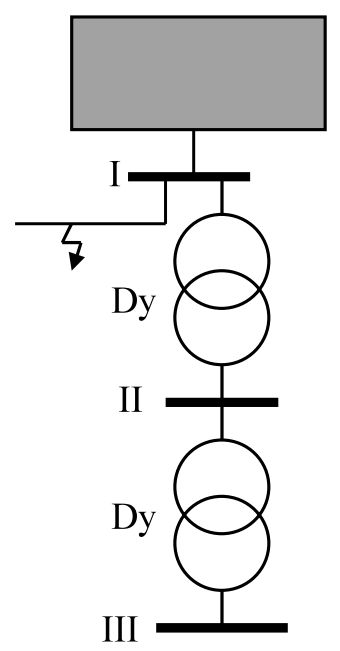

Figura 3.5: Huecos a diferentes niveles de tensión debidos a diferentes tipos de faltas [2].

entre las fases a y c); Cc (hueco entre las fases a y b). Esta clasificación se ha llevado a cabo mediante una notación consecuente con la clasificación ABC. Así, para $F=E_{1}$ y $V=V^{*}, 3.3$ y 3.4, son idénticas al tipo D y tipo $\mathrm{C}$ respectivamente, a como se han definido en la tabla 3.1. La descripción de esta metodología no se ha hecho con mayor detalle al no ser una parte fundamental de desarrollos posteriores. Una descripción con mayor detalle de esta metodología se puede encontrar en las referencias citadas en esta sección.

\subsubsection{Caracterización mediante componentes dq.}

Tal y como se ha comentado en el apartado anterior, tradicionalmente los huecos de tensión se han caracterizado de forma simplificada por su duración y su magnitud, entendida esta última como la máxima reducción de tensión eficaz sufrida por las fases. Esta caracterización proporciona información relevante de la perturbación, aunque en la mayoría de ocasiones es insuficiente para identificar adecuadamente la severidad y la evolución del evento. Así, y además de la caída de tensión eficaz, hay también un desplazamiento del ángulo de fase asociado con él [27]. De acuerdo con aportaciones precedentes, este ángulo de fase puede influir de manera notable en disparos intempestivos de equipos [109]. Además, al considerar sólo la caída de tensión eficaz producida por la fase más afectada por el hueco, esta caracterización sería adecuada para perturbaciones equilibradas, mientras que dejaría de aportar información sobre la evolución del resto de fases para el caso de eventos desequilibrados, estuviesen o no sometidas a alteraciones en su tensión. Sin 
embargo, y de acuerdo con la totalidad de los estudios realizados, la mayoría de los huecos de tensión son desequilibrados [27]. Por todo ello, resultaría más adecuada la representación y caracterización de perturbaciones mediante técnicas que tuviesen en cuenta la evolución de todas las fases a lo largo del hueco de tensión y, al mismo tiempo, permitiesen de forma sencilla visualizar la severidad y trayectoria del evento. De acuerdo con la bibliografía consultada y atendiendo a contribuciones previas, se propone el empleo de la transformación de Park como herramienta para la visualización y caracterización de huecos de tensión, englobando esta transformación la información relativa a todas las fases y no sólo a la fase más afectada por el hueco. Así, la transformación de Park o d-q convierte las componentes $a b c$ del sistema trifásico a otro sistema de referencia $d q 0$. El objetivo de la transformación consiste en convertir los valores trifásicos $a b c$ en variables senosoidalmente en el tiempo, a valores constantes $d q 0$. Para esto se parte del sistema de referencia estacionario o transformada de Clarke.

$\mathrm{Si}$ se considera un sistema trifásico simétrico y equilibrado, se puede demostrar que las corrientes y tensiones trifásicas del sistema se pueden representar mediante un vector espacial de módulo constante que gira a velocidad constante en el plano $\prod$ [110]. La transformada de Clarke consiste en multiplicar el vector de tensiones $(\mathrm{a}, \mathrm{b}, \mathrm{c})$ por la matriz de transformación de Clarke y da como resultados los ejes $0, \alpha$ y $\beta$. Se define como [111,

$$
\left(\begin{array}{l}
x_{\alpha}(t) \\
x_{\beta}(t) \\
x_{0}(t)
\end{array}\right)=\frac{2}{3}\left(\begin{array}{ccc}
1 & -\frac{1}{2} & -\frac{1}{2} \\
0 & \frac{\sqrt{3}}{2} & -\frac{\sqrt{3}}{2} \\
\frac{1}{2} & \frac{1}{2} & \frac{1}{2}
\end{array}\right)\left(\begin{array}{l}
v_{a}(t) \\
v_{b}(t) \\
v_{c}(t)
\end{array}\right)
$$

donde el coeficiente $\frac{2}{3}$ se usa para conservar las magnitudes entre las dos coordenadas del sistema.

Las dos primeras componentes de la transformada de Clarke forman el vector espacial 3.5 y la tercera componente representa la tensión a la secuencia cero 3.6 .

$$
\begin{gathered}
\vec{x}(t)=x_{\alpha}(t)+j x_{\beta}(t)=\frac{2}{3}\left[\begin{array}{lll}
1 & e^{j \frac{2 \pi}{3}} & e^{j \frac{4 \pi}{3}}
\end{array}\right]\left[\begin{array}{l}
v_{a}(t) \\
v_{b}(t) \\
v_{c}(t)
\end{array}\right] \\
x_{0}(t)=\frac{1}{3}\left(v_{a}(t)+v_{b}(t)+v_{c}(t)\right)
\end{gathered}
$$

En el caso de un sistema de componentes simétricos el valor de $x_{0}(t)$ es igual a cero.

A partir de este resultado se puede deducir que la tensión trifásica $\mathrm{v}(\mathrm{t})$ sigue una trayectoria circular de radio $\rho$ y velocidad angular constante $\omega$ sobre el plano $\prod$, tal y cómo muestra la figura 3.6 .

El vector espacial contiene toda la información de las tres fases, incluido el estado estable desequilibrado, distorsión de onda armónica y componentes transitorios [112]. 


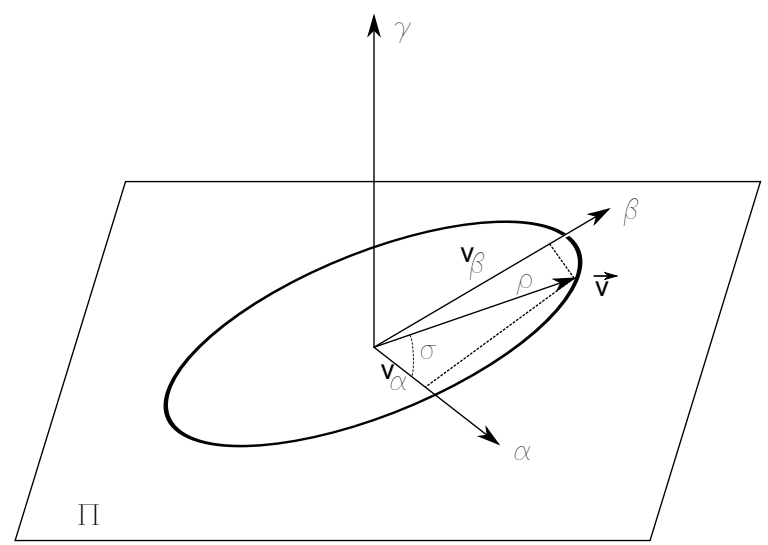

Figura 3.6: Representación de la trayectoria del vector v(t) sobre el plano $\prod$.

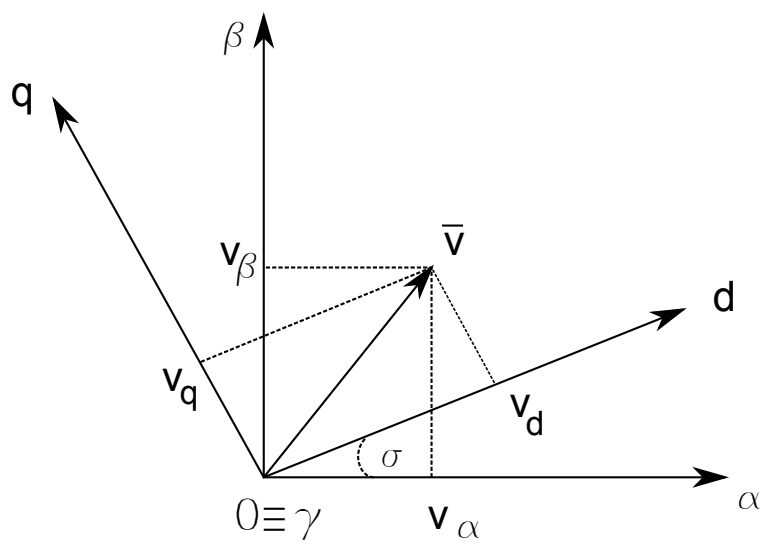

Figura 3.7: Cambio de base de los ejes $\alpha-\beta-\gamma$ a los ejes $d q 0$.

Una vez hecho el cambio de base, las tensiones del sistema girarán con velocidad angular constante $\omega$ sobre el plano $\prod$, definido por los ejes $\alpha-\beta$. No obstante, lo que realmente se persigue es que el vector espacial de tensión permanezca fijo en una determinada posición, de modo que en régimen permanente sea un vector constante. Esto se consigue definiendo un sistema de referencia que gire sobre el plano $\prod$ a la misma velocidad $\omega$ que el vector espacial.

La figura 3.7 muestra cómo se realiza el cambio de ejes sobre el plano $\prod$. La matriz de transformación de Park, 3.7 [113, permite el cambio de 


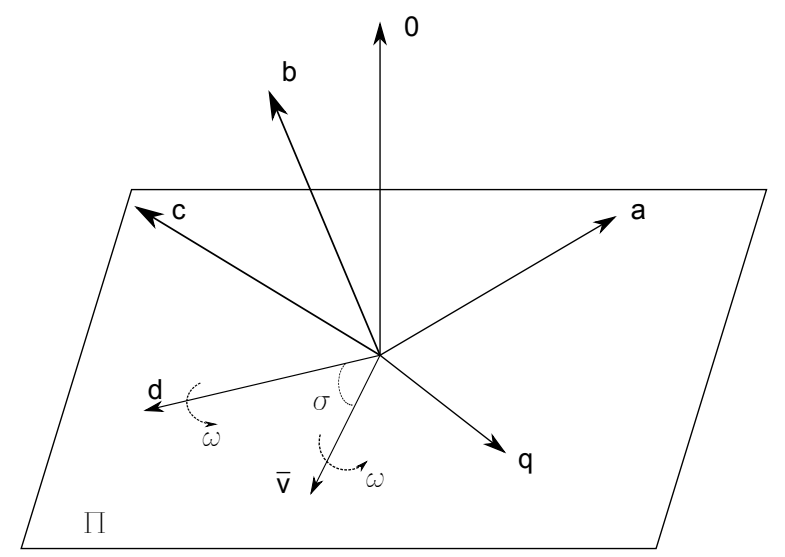

Figura 3.8: Representación del vector espacial v en la nueva base $d q 0$.

base $\alpha-\beta-\gamma$ a $d q 0$.

$$
\begin{aligned}
{\left[\begin{array}{l}
v_{d} \\
v_{q} \\
v_{0}
\end{array}\right]=} & {\left[\begin{array}{l}
k_{d} \\
k_{q} \\
k_{0}
\end{array}\right]\left[\begin{array}{ccc}
\cos \left(\theta_{s}(t)\right) & \cos \left(\theta_{s}(t)-\frac{2 \pi}{3}\right) & \cos \left(\theta_{s}(t)+\frac{2 \pi}{3}\right) \\
\operatorname{sen}\left(\theta_{s}(t)\right) & -\operatorname{sen}\left(\theta_{s}(t)-\frac{2 \pi}{3}\right) & -\operatorname{sen}\left(\theta_{s}(t)+\frac{2 \pi}{3}\right) \\
\frac{1}{2} & \frac{1}{2}
\end{array}\right]\left[\begin{array}{l}
v_{a} \\
v_{b} \\
v_{c}
\end{array}\right] } \\
v_{d}(t) & =k_{d}\left[v_{a}(t) \cdot \cos \left(\theta_{s}(t)\right)+v_{b}(t) \cdot \cos \left(\theta_{s}(t)-\frac{2 \cdot \pi}{3}\right)+\right. \\
& \left.+v_{c}(t) \cdot \cos \left(\theta_{s}(t)+\frac{2 \cdot \pi}{3}\right)\right] \\
v_{q}(t) & =k_{q}\left[-v_{a} \cdot \operatorname{sen}\left(\theta_{s}(t)\right)-v_{b} \cdot \operatorname{sen}\left(\theta_{s}-\frac{2 \cdot \pi}{3}\right)-\right. \\
& \left.-v_{c}(t) \cdot \operatorname{sen}\left(\theta_{s}+\frac{2 \cdot \pi}{3}\right)\right]
\end{aligned}
$$

Los ejes d-q giran sobre el plano $\prod$ a la misma velocidad angular $\omega$ que el vector espacial v, tal y como muestra la figura 3.8 .

En [3] se propone una clasificación y caracterización de huecos trifásicos por la metodología de vector espaciales, como una evolución a las clasificaciones anteriores. Atendiendo a la transformada de Clarke y asumiendo que la tensión se mantiene como onda senosoidal a lo largo de la falta, puede hacerse uso de la fórmula de Euler para obtener la expresión 3.9 .

$$
\vec{x}=x_{p} e^{j w t}+x_{n} e^{-j w t},
$$

donde $x_{p}$ y $x_{n}$ son valores complejos.

La figura 3.9 recoge la representación del vector espacial como suma de dos vectores de la misma velocidad angular y sentidos opuestos de giro, módulos constantes pero de diferente magnitud y diferente desfase inicial. 


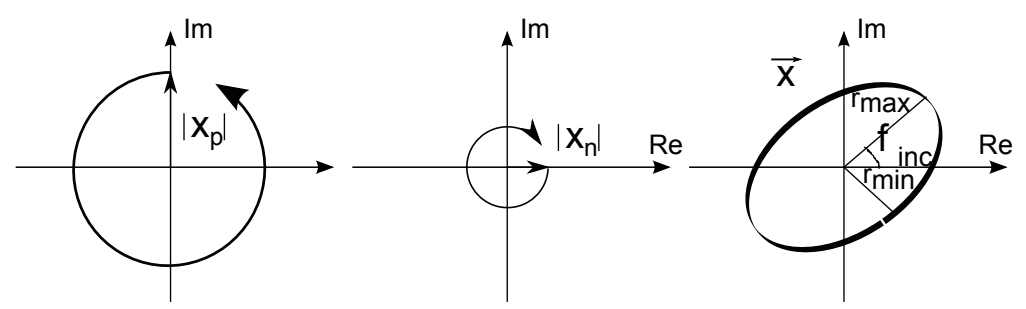

Figura 3.9: Descomposición del vector espacial como suma de dos vectores de módulo y velocidad angular constantes [3].

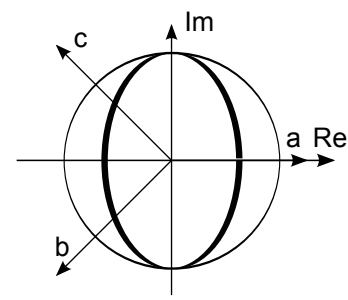

a)

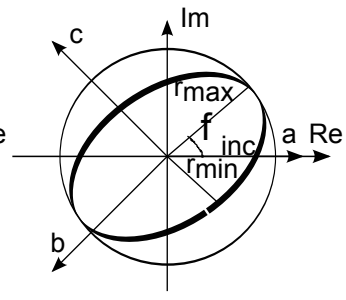

b)

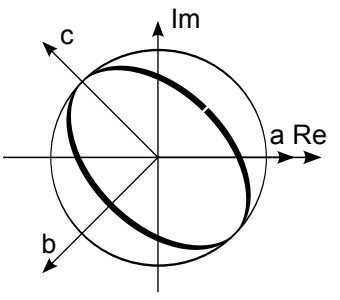

c)

Figura 3.10: Vector espacial para un hueco manofásico tipo B, en las fases a y c respectivamente [3].

La figura 3.10 representa huecos monofásicos a través de la elipse correspondiente. Tal y como puede apreciarse, la posición del eje mayor de la elipse rota en ángulos de $120^{\circ}$ según la fase que esté afectada por el hueco monofásico. Así, y para huecos monofásicos, la posición de los ejes de la elipse indicarían la fase afectada por el hueco. Además, en este caso particular, el eje mayor de la elipse coincide con el diámetro de la circunferencia correspondiente a la situación previa antes del hueco.

\subsection{Propuesta de Inclusión de Severidad de Hueco.}

\subsubsection{Plantemiento General.}

Habitualmente, los equipos de medida de calidad de suministro calculan el voltaje eficaz estimando un valor por ciclo, permitiendo detectar eventos cuando la tensión cae por debajo de un valor umbral [94]. Los equipos toman el valor más bajo de la tensión eficaz como la magnitud de tensión residual. Esta magnitud se expresa como un porcentaje de la tensión nominal [94]. Ejemplos reales de medida y representación de huecos de tensión se pueden ver en las figuras 3.11, 3.12, 3.13 y 3.14. En todos estos casos, las figuras etiquetadas como (a) muestran los valores instantánesos de tensión recogidos 


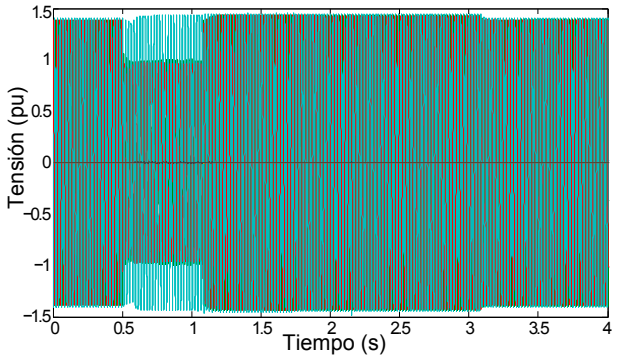

(a) Valores de tensión instatáneos

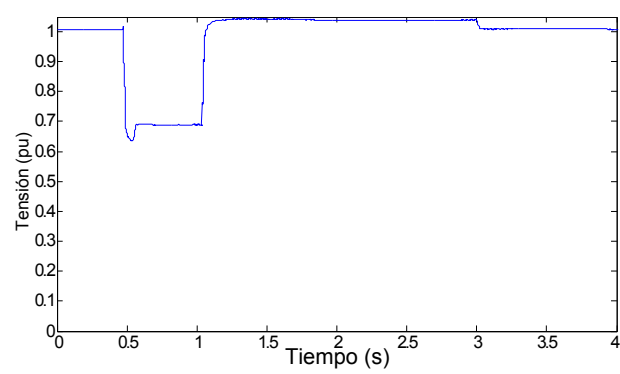

(b) Valores estimados de rms

Figura 3.11: Ejemplo de hueco bifásico medido en un huerto solar fotovoltaico.

directamente de un equipo de medida de calidad de potencia; mientras que las figuras etiquetadas como (b) recogen el valor de tensión eficaz estimada mediante la expresión 3.1 para la fase más afectada por la perturbación. Estos huecos son ejemplos reales de la heterogeneidad de eventos que pueden producirse al realizar medidas de campo; lo que afianza la necesidad de estimar el valor eficaz con un paso igual al tiempo de muestreo, y no determinar un valor por ciclo de onda de tensión.

En lo que respecta a la caracterización de los huecos de tensión, tal y como ya se ha comentado en este documento, las variables que habitualmente se han considerado como propicias para representar un conjunto de perturbaciones han sido el intervalo de máxima duración del hueco y la mayor profundidad de todas las fases [94], [2], 27], [101]. Sin embargo, y atendiendo a la gran cantidad de casos prácticos analizados, puede afirmarse que este conjunto simplificado de parámetros no recoge fielmente la severidad y evolución de los eventos. Así, y a modo de ejemplo, las figuras 3.11 y 3.12 muestran dos huecos de tensión con la misma profundidad, pero con distinta duración. La figura 3.13 muestra un hueco con muy poca tensión residual, pero durante un periodo de tiempo muy pequeño, mientras que la figura 3.14 recoge un hueco con una gran tensión residual durante un periodo muy largo de tiempo. De hecho, estas situaciones en las que se detectan sobretensiones transitorias unidas a huecos de tensión deberían ser estudiadas para su posible inclusión en los actuales requerimientos de conexión para instalaciones renovables conectadas a red. Así, no sólo deberían incluirse las disminuciones de tensión eficaz propias de estas perturbaciones, sino también los aumentos puntuales y transitorios de tensión que en ocasiones van a aparejados a estos eventos.

A modo de conclusión, y dada la falta de uniformidad en los eventos reales recogidos a lo largo de distintas campañas de medida, sería interesante definir nuevas variables que completen la caracterización de las perturbaciones en términos de su severidad. Como se ha visto, hay huecos que tienen una misma 


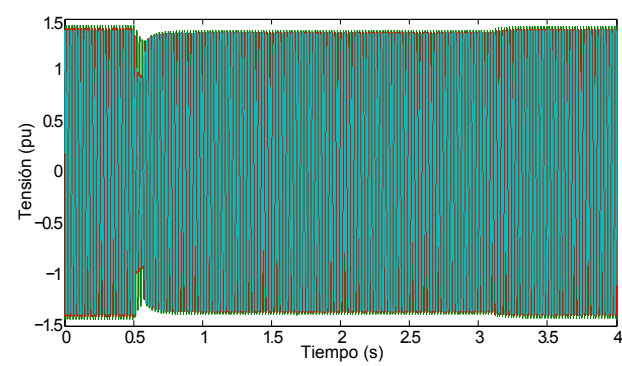

(a) Valores de tensión instantáneos

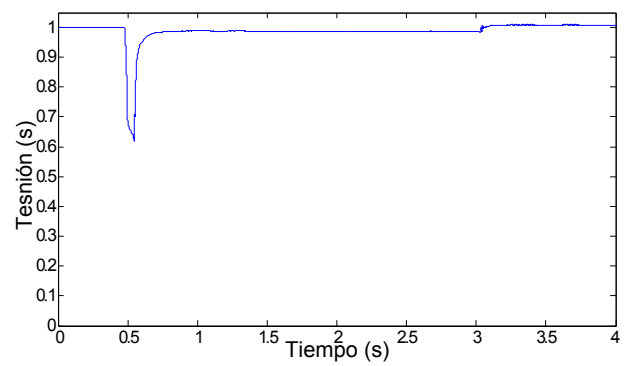

(b) Valores estimados de rms

Figura 3.12: Ejemplo de hueco trifásico medido en un huerto solar fotovoltaico.

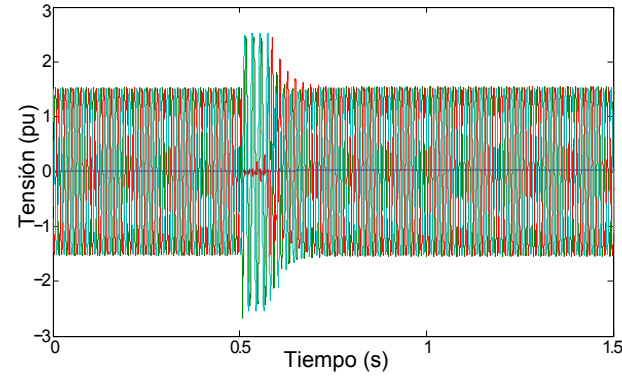

(a) Valores de tensión instantáneos

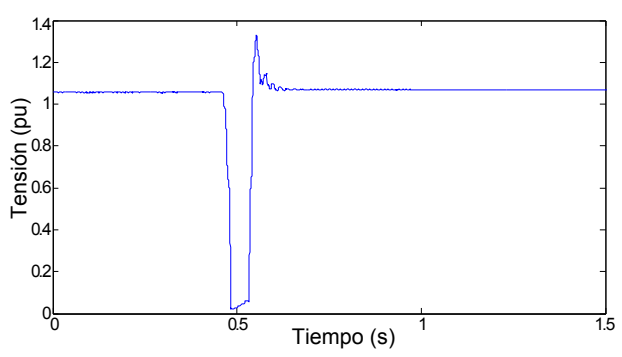

(b) Valores estimados de rms

Figura 3.13: Ejemplo de hueco monofásico medido en una parque eólico.

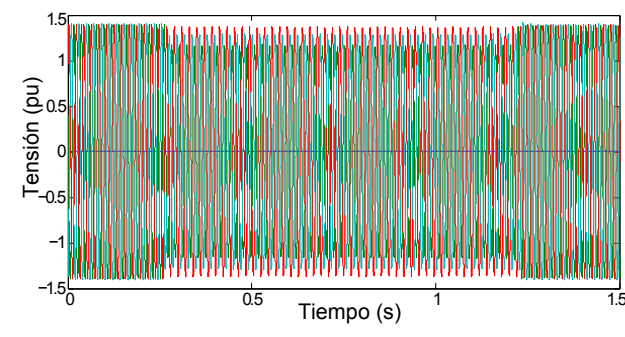

(a) Valores de tensión instantáneos

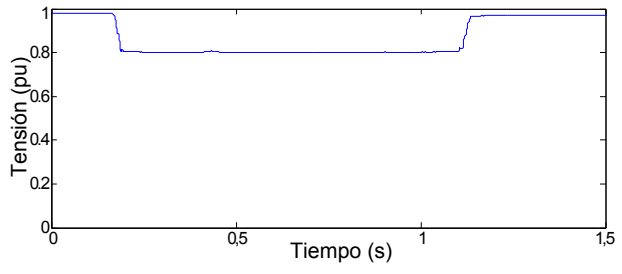

(b) Valores estimados de rms

Figura 3.14: Ejemplo de hueco trifásico medido en un parque eólico. 
tensión residual y un tiempo de duración similar, y sin embargo presentan evoluciones totalmente distintas, lo que lleva a comportamientos diferentes para los equipos que han de soportar estas perturbaciones. Así pues, se hace necesario cuantificar no sólo el valor de la tensión residual, como mínimo de tensión eficaz presente en un hueco, sino también el intervalo de tiempo que la perturbación permanece cercana a este valor, como medida de la severidad del hueco. De hecho, estudios previos como los presentados en [33] y [114] demuestran cómo las cargas con etapa electrónica son sensibles no sólo a la profundidad y duración del hueco, sino al tiempo alrededor del mínimo de tensión. Para ello se incluyen en los trabajos un extenso conjunto de ensayos de laboratorio donde se han generado perturbaciones dentro de un amplio rango de tensiones residuales, duraciones y puntos de inicio dentro de la onda senoidal.

\subsubsection{Propuesta de severidad en estudios basados en RMS.}

En línea con lo comentando en la sección anterior, y a tenor tanto de la disparidad de trayectorias como de la sensibilidad de las cargas a la dinámica de la perturbación, se hace necesario aumentar el número de parámetros con los que caracterizar el hueco, inicialmente limitados a la duración total y a la tensión residual. La figura 3.15, muestra un claro ejemplo de dos huecos de distinta evolución cuyos parámetros característicos en términos de duración y tensión mínima son similares. Sin embargo, la permanencia de cada uno de ellos alrededor del valor mínimo difiere considerablemente, lo que hace que el esfuerzo a realizar para mantener conectados a red los equipos sea muy distinto. Así pues, para caracterizar la severidad de los huecos de tensión se propone incluir como parámetro adicional de estudio un intervalo de tiempo al rededor del valor del mínimo, no sólo desde el punto de vista del valor residual de la tensión, sino también desde el periodo de tiempo que el hueco está cerca de ese valor de tensión residual. La inclusión de este tercer parámetro para identificar perturbaciones permitiría igualmente una visualización y comparación de un conjunto de huecos, ya que ahora cada uno de ellos podría definirse, dentro de unos ejes duración-tensión residual, mediante una esfera cuyo radio fuese función del intervalo de tiempo que la perturbación permaneciese cercana al valor mínimo. En concreto, en este documento de tesis se ha considerado como umbral el instante en el que la tensión se recupere un $10 \%$ por encima del valor mínimo alcanzado. Esta propuesta extendida de caracterización de huecos ha sido aplicada a diferentes conjuntos de perturbaciones, permitiendo una visualización mayor de las características de los huecos medidos y aportando una medida de la severidad de los mismos basada no sólo en la duración total y la tensión mínima alcanzada, sino en el intervalo de tiempo que el hueco permanece próximo a este valor mínimo, lo cual también aporta una idea adicional de los tiempos empleados por el mismo en el inicio y en la recuperación de la tensión. 


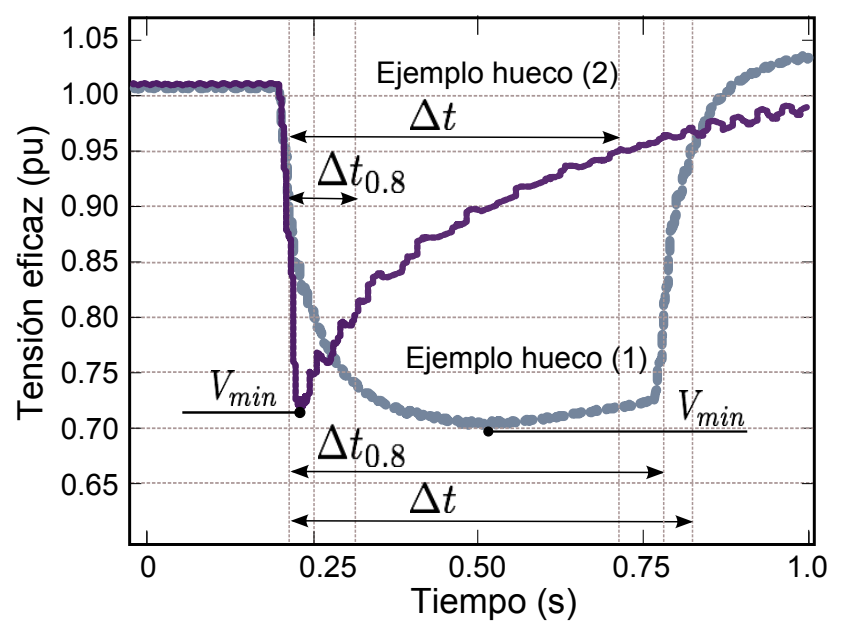

Figura 3.15: Comparativa huecos de tensión.

\subsection{Nuevas Propuestas de Caracterización: Comparativa ante Requerimientos.}

Si se hace un repaso a la normativa emitida por distintos países, tanto del ámbito europeo como internacional, en la mayoría de los casos se han definido los requerimientos de conexión ante perturbaciones dentro de unos ejes tensión eficaz-tiempo. Sobre ellos se han representado los límites permitidos para la evolución de la tensión eficaz en la fase más afectada por la falta. Actualmente existe una gran diversidad de estos requerimientos, tanto en lo que respecta a los valores mínimos que las unidades de generación han de soportar, como en los intervalos de tiempo máximo fijados por los distintos operadores del sistema. Este abanico de normativas se encuentra recogido en la figura 3.18 - ya comentada en el capítulo anterior-, donde se han superpuesto a modo de ejemplo las normativas vigentes para parques eólicos conectados a red.

Con el fin de caracterizar estas normativas con un número reducido de parámetros, la presente tesis propone la linealización mediante una definición de tramos de la trayectoria seguida por la tensión eficaz, de forma que puedan identificarse un conjunto reducido de segmentos. Estas longitudes representan, según el tramo recorrido, o bien variaciones en el valor de tensión eficaz o bien intervalos de tiempo transcurridos alrededor de un mismo valor de tensión. Con ello se propone identificar los requerimientos de conexión no ya con un perfil de tensiones admisibles dentro de unos ejes tensión eficaz-tiempo, sino como un conjunto de cuatro segmentos perfectamente identificables. En concreto, para la normativa vigente en el sistema eléctrico español, la figura 3.17 recoge tanto la definición de estos puntos como los segmentos limitados 


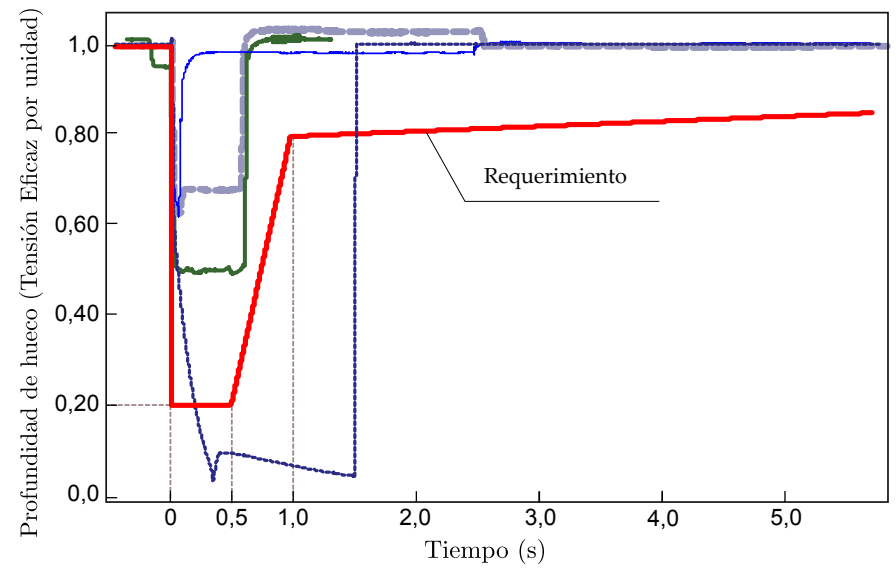

Figura 3.16: Comparativa de perturbaciones reales frente a normativa de conexión española para instalaciones renovables dada en [4].

por dichos puntos. En virtud de la figura 3.18 esta propuesta es extrapolable al resto de países donde existe normativa de conexión ante perturbaciones. Más aún, actualmente está en estudio un borrador para unificar criterios de conexión promovido por ENTSO-E (ver apartado 2.4.2, el cual también se basa en definir los límites de conexión a través de una trayectoria máxima permitida para la tensión eficaz de la fase más afectada por la perturbación. En este caso, también sería de aplicación la propuesta de segmentos aquí descrita, lo cual permite extender esta propuesta a borradores en estudio a fecha de realización de esta tesis.

La figura 3.19 muestra el conjunto de longitudes propuestas $\left(\begin{array}{lll}l_{1} & \text { a } & l_{4}\end{array}\right)$, aplicados sobre los requerimientos de conexión. En este caso se toma como ejemplo los requerimientos de conexión españoles para las unidades de generación conectadas a la red y sometidas a huecos de tensión. Como se deduce de la figura, el conjunto de longitudes propuestas mantiene una relación directa con los límites requeridos para las tensiones eficaces y duraciones, dividiendo cada trayectoria de tensión eficaz en segmentos que caractericen severidad y pendientes máximas permitidas por la normativa. Estas longitudes son por lo tanto el fundamento de una representación relevante de un hueco de tensión. Así pues, se propone representar y caracterizar los eventos medidos mediante estas longitudes, que están claramente relacionadas con los intervalos de tiempo y los límites de tensión eficaz. Además, la severidad de las perturbaciones está también incluida como el valor $l_{2}$, representando el intervalo de tiempo relativo alrededor del valor mínimo de la tensión eficaz - tensión eficaz residual-. Se considera un intervalo de tiempo adicional desde el inicio de la perturbación hasta que la tensión eficaz se recupera sobre el $0,8(\mathrm{pu})$, etiquetada como $\Delta t_{0,8}$. En concordancia con los actuales 


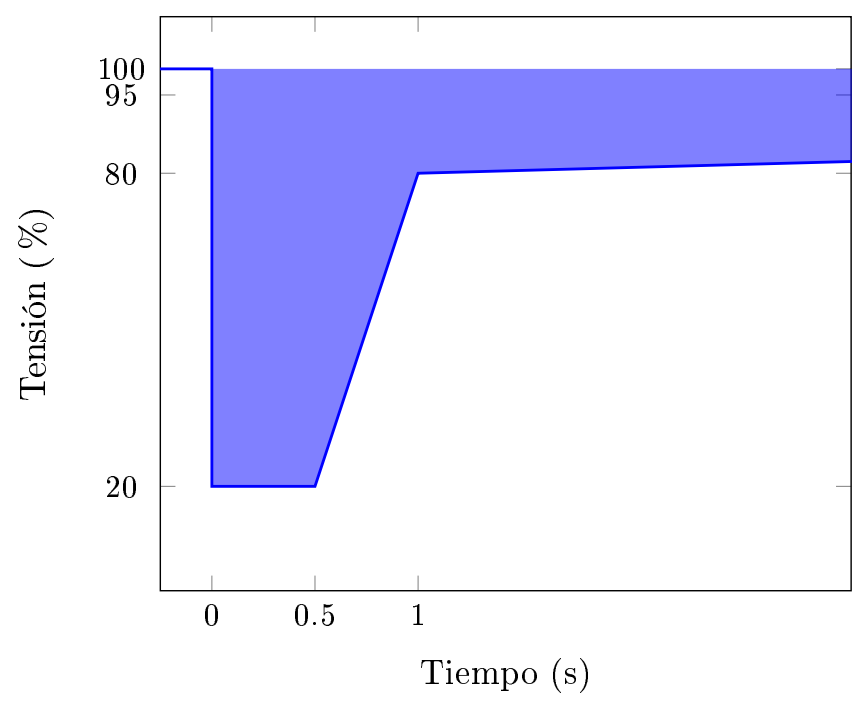

Figura 3.17: Curva española de tensión-tiempo que define el área del hueco de tensión en el punto de conexión a la red que debe ser soportado por la instalación.

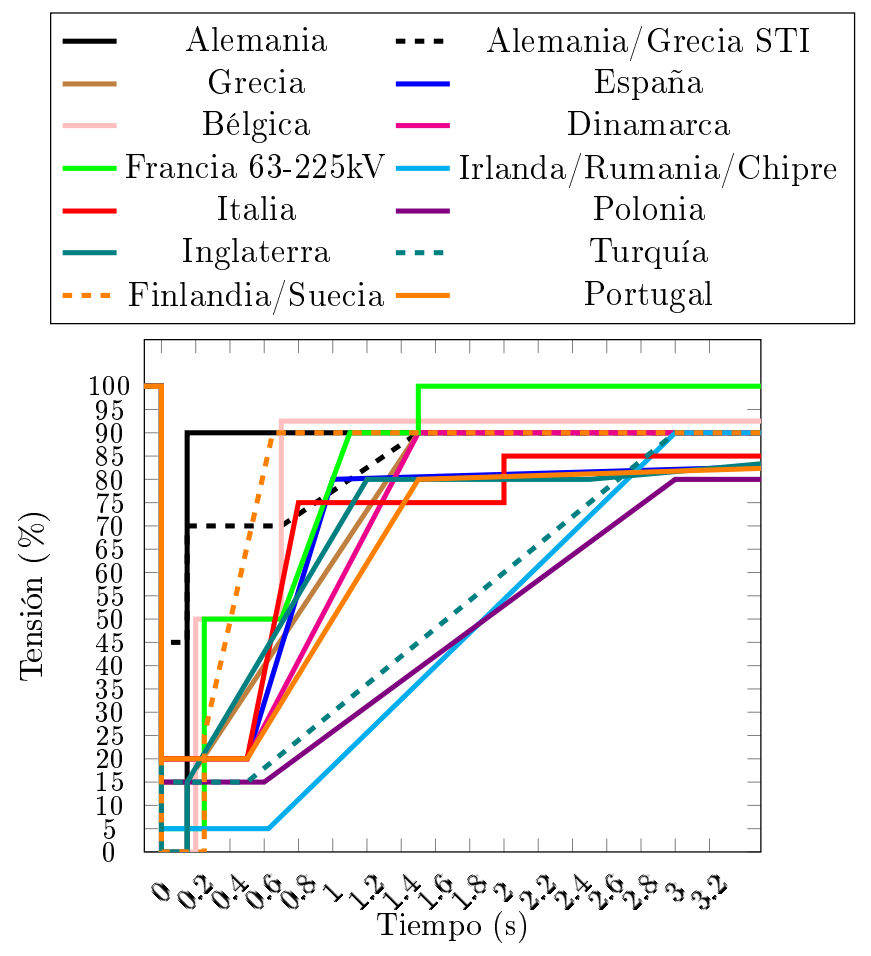

Figura 3.18: Requerimientos de conexionado para parques eólicos: Comparativa de las curvas del valor límite de tensión eficaz en el punto de conexión a la red [1]. 


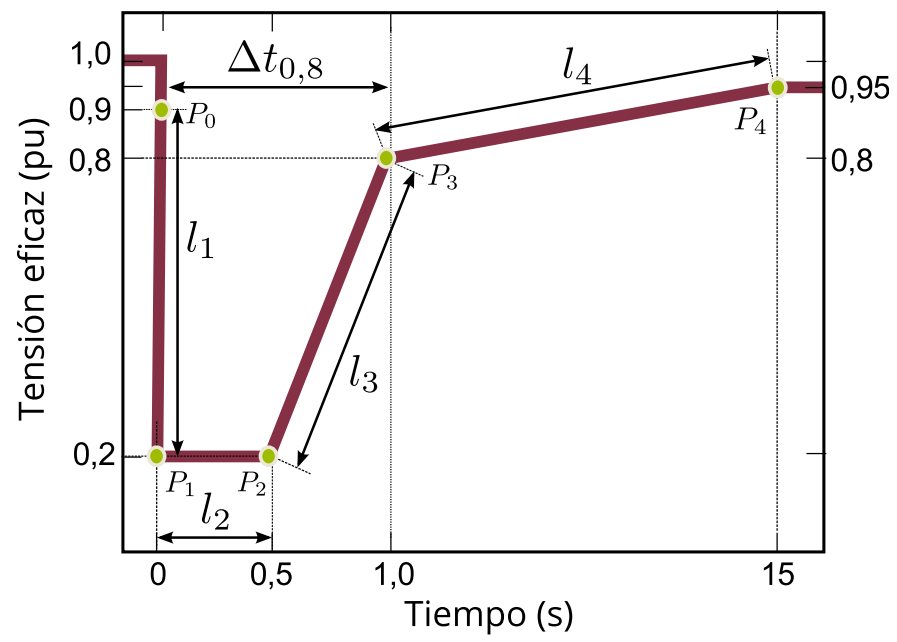

Figura 3.19: Caracterización de perturbaciones mediante conjunto de longitudes. Aplicación a requerimientos del sistema eléctrico español.

requerimientos de conexión, el ratio entre este intervalo de tiempo parcial y la duración global del evento da una estimación de cómo de aguda es la perturbación.

Un ejemplo de esta propuesta de caracterización se muestra en la figura 3.20, donde se identifica una perturbación real medida en un parque eólico español por el correspondiente conjunto de longitudes $\left(l_{1}\right.$ a $\left.l_{4}\right)$. Los requerimientos de conexión español están también incluidos en la figura, como un intento preliminar para comparar-en términos de tensión eficaz residual, tiempo de duración y severidad - los datos medidos y los perfiles de tensión permitidos dados por este requerimiento actual. Para fijar los valores de las 4 longitudes se ha tenido en cuenta los puntos más característicos de las perturbaciones:

- $l_{1}$ se define como 0,9- $V_{\text {min }}$. Es la longitud del segmento $P_{0} P_{1}$.

- $l_{2}$ es el tiempo que permanece alrededor de $V_{\text {min }}$ : es el tiempo que tarda la tensión eficaz en recuperar el $10 \%$ alrededor del valor $V_{\text {min }}$. Está asociado al segmento horizontal $P_{1} P_{2}$.

- $l_{3}$ es la longitud del segmento que va desde el punto $P_{2}$ hasta el punto donde $\mathrm{V}$ recupera el 0,8 ( $P_{3}$ en la figura). En este caso cuando $1,1 V_{\min }>0,8, l_{3}$ se establece en 0 .

- $l_{4}$ es la longitud de $P_{2}$ al punto $P_{4}$ donde $V=0,95$ y la tensión se considera que se ha recuperado completamente. 


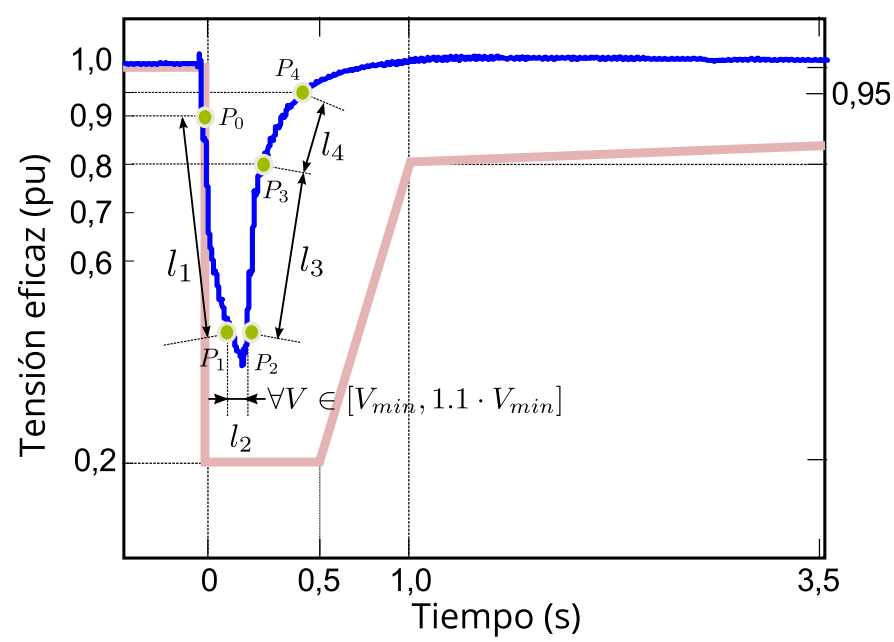

Figura 3.20: Caso práctico de identificación de longitudes. Comparativa ante requerimientos del sistema eléctrico español.

Nótese que la severidad del hueco de tensión no está cuantificada como la tensión eficaz residual, sino se considera como el intervalo de tiempo $\left(l_{2}\right)$ hasta que la tensión recupera el $10 \%$ de su valor mínimo. Esta propuesta está en línea con la evolución típica de la mayoría de los perfiles reales de tensiones eficaces bajo la presencia de faltas, las cuales presentan claramente una gran diversidad y para las cuales solo unas pocas muestras son medidas normalmente para valores mínimos de tensión eficaz. Este intervalo de tiempo relativo aporta un tiempo realista de duración alrededor del valor del mínimo de la tensión eficaz de perturbaciones reales. Más información sobre este parámetro adicional y ejemplos de instalaciones reales de energía FV se pueden encontrar en [115. 



\section{Capítulo 4}

\section{Identificación de Patrones en Conjuntos de Huecos de Tensión: Comparativa Frente a Requerimientos de Conexión.}

\footnotetext{
Resumen: A partir del conjunto de longitudes con las que describir la trayectoria del valor eficaz a lo largo de la perturbación, este capítulo tiene como objetivo la visualización y caracterización — de manera ágil y simple- de un conjunto de perturbaciones. Para ello se aplica un análisis estadístico por componentes principales con el objetivo de reducir la dimensión del cojunto de longitudes, sin pérdida significativa de información. En paralelo a este proceso, se aporta una metodología de identificación de un número reducido de patrones que resuman, en promedio, todas las trayectorias de tensión eficaz presentes en un grupo de eventos. Esta estimación de patrones ofrece una herramienta práctica y visual para establecer comparaciones entre dichos patrones y los requerimientos de conexión de los operadores del sistema. Con ello, se ofrece un análisis global de las diferencias y similitudes entre los límites permitidos por la normativa y las evoluciones de tensión más habituales de un conjunto inicial de perturbaciones medidas en emplazamientos concretos.
} 


\subsection{Planteamiento de la Problemática.}

\subsubsection{Métodos de Caracterización para Conjuntos de Huecos de Tensión. Estado del Arte.}

Tal y como se ha comentado en el capítulo anterior, los huecos de tensión suelen habitualmente caracterizarse a partir de la representación de los perfiles de tensiones eficaces de cada fase. En concreto, se escoge la trayectoria de la tensión eficaz correspondiente a la fase más afectada por la perturbación. Esta representación es la más empleada debido a que los requerimientos de conexión a red se han desarrollado usualmente en base a la evolución de tensiones eficaces. A la hora de representar un conjunto de perturbaciones de manera conjunta, la mayoría de aportaciones han propuesto escoger un número reducido de parámetros con los que identificar cada evento. Así, los perfiles de tensión eficaz se han resumido habitualmente mediante la estimación de la tensión residual y la duración total de la perturbación. A partir de estos parámetros, las caracterizaciones y representaciones de conjuntos de medidas de huecos de tensión más empleadas se han basado en tablas de densidad [116], [117], [96], diagramas de colores [117], [118], [28] o gráficos de dispersión [5]. A continuación pasan a comentarse cada una de estas soluciones.

- Las tablas de densidad muestran un análisis resumido de eventos para la caracterización de una campaña de medidas. Para ello se realiza una distribución de huecos de tensión atendiendo a los valores de tensión residual y de tiempo total de la perturbación. Así, cada celda de la tabla muestra el número de eventos correspondiente a un rango concreto de tensión residual y de tiempo global de duración de la falta. La fila inferior suele reservarse para resumir el total de los eventos clasificados según su duración global, mientras que en la columna de la derecha se agrupan en función del rango de tensión residual alcanzado durante la perturbación.

- Los mapas de colores ofrecen diferentes formas de representación. Normalmente se emplean diagramas tridimensionales para representar gráficamente los eventos, asignando a cada las variables tensión eficaz residual, duración del hueco y número de eventos respectivamente. Una vía alternativa para sintetizar y mostrar las perturbaciones se encuentra propuesta en [119]. Este tipo de representación, llamado mapa de colores, clasifica las perturbaciones en sectores rectangulares. A cada sector se le asigna un color, dependiendo del número de eventos que se registren dentro de un rango de tiempo total de duración y de tensión residual alcanzada durante el evento. De esta manera, los mapas de colores muestran una representación general de los eventos recogidos 
y de la distribución de éstos en base a unos rangos predefinidos de tensión residual y de tiempo de duración.

- Los gráficos de dispersión suponen una vía alternativa y ampliamente propuesta para caracterizar un elevado número de eventos. En este caso, los eventos son representados por un punto dentro de unos ejes que recogen la magnitud de tensíón residual y duración. Un ejemplo de un gráfico de dispersión se puede ver en la figura 4.1. El principal inconveniente que presenta este tipo de caracterización es común a los métodos anteriores, y es la limitación de información que aportan para cada hueco de tensión. Un avance en este campo se presenta en [5], donde se parte de los gráficos de dispersión pero introduciendo una diferenciación por tamaños en los marcadores del gráfico. El tamaño del marcador es proporcional al área generada como consencuencia de la disminución de la tensión eficaz, calculada en voltios-segundo. Esta información ayuda a diferenciar perturbaciones en base a una estimación de la trayectoria generada por la tensión eficaz. Los autores describen el área por la perturbación como una medida de la pérdida de energía durante el hueco de tensión. Este artículo es de las pocas contribuciones encontradas en las que se tiene en cuenta toda la envolvente creada por el hueco, dando así importancia a la trayectoria de la tensión eficaz durante la perturbación, y no sólo a los parámetros de duración total y valor mínimo alcanzado. Sin embargo, esta contribución no resuelve el problema de la representación y análisis conjunto de un grupo de perturbaciones, presentando igualmente el inconveniente del solapamiento de información, y dificultando así la toma de decisiones y la deducción clara de conclusiones. La figura 4.2 muestra un ejemplo de representación de este tipo de caracterización.

\subsubsection{Evaluación de Métodos de Caracterización mediante Campañas de Medidas.}

A partir de los métodos de caracterización comentados en el apartado anterior, se incluye ahora una evaluación y comparativa de los mismos para mostrar sus características y posibles mejoras. Con tal fin se hará uso de un elevado número de perturbaciones reales recogidas en instalaciones renovables conectadas al sistema eléctrico español. Estos datos son fruto de campañas de medida principalmente promovidas por el Instituto de Energías Renovables de la Universidad de Castilla La Mancha. En concreto se han llevado a cabo actuaciones sobre: (i) dos plantas fotovoltaicas y (ii) un parque eólico. Estas campañas de medidas se han realizado a lo largo de los últimos años al amparo de diferentes proyectos nacionales y regionales en los que ha participado el grupo de investigación donde se ha desarrollado la presente tesis. 


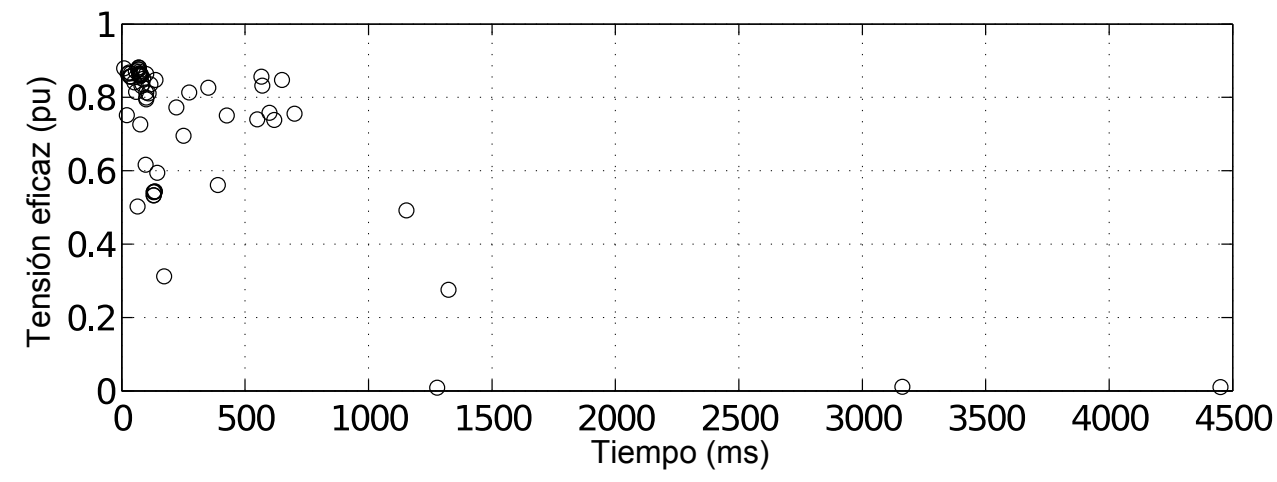

(a) Eventos (duración leg $4500 \mathrm{~ms}$ )

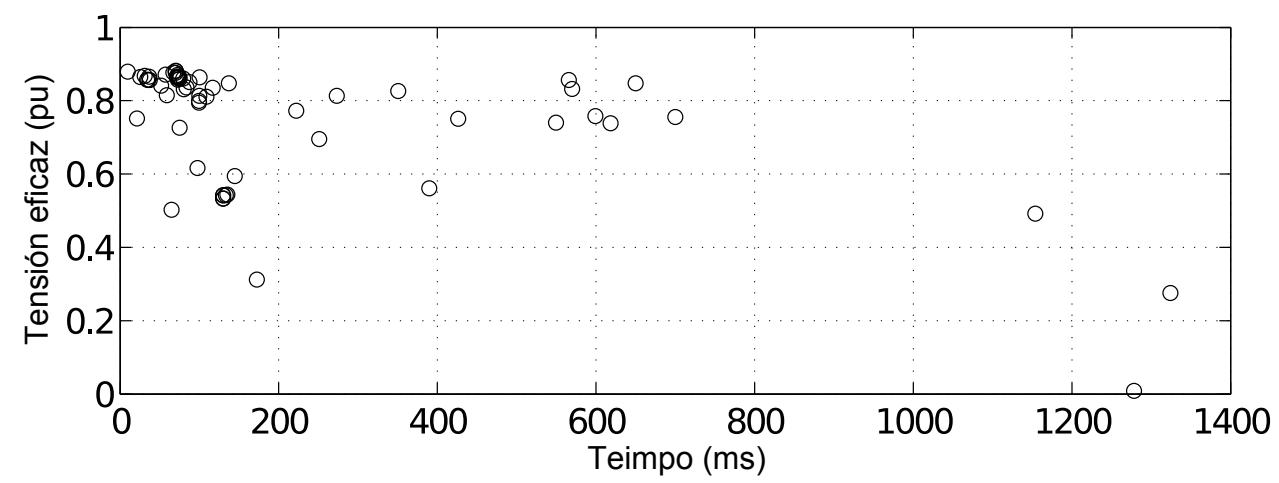

(b) Eventos (duración leg $1400 \mathrm{~ms}$ )

Figura 4.1: Ejemplo gráfico de dispersión.

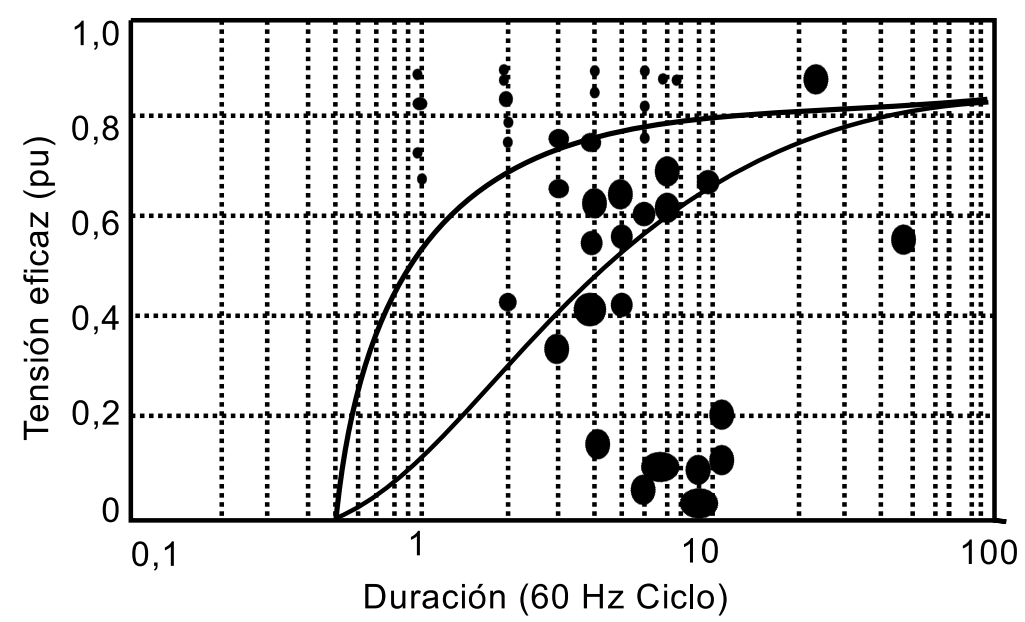

Figura 4.2: Gráfico de dispersión incluyendo el área de la perturbación. (Fuente: [5]). 
En lo que respecta a instalaciones fotovoltaicas, se han realizado campañas de medidas en dos huertos solares ubicados en zonas con un elevado nivel de radiación solar: Castilla-La Mancha y la Región de Murcia. Ambos emplazamientos están situados al sureste de España, donde se están desarrollando proyectos vinculados con el aprovechamiento de energía solar y donde han proliferado instalaciones fotovoltaicas a lo largo de los últimos años. La instalación fotovoltaica localizada en la Región de Castilla-La Mancha está constituida por dos instalaciones con una potencia nominal instalada de 5 MW. Una de las instalaciones comenzó a funcionar en 2010. Se trata de una instalación fija de $4 \mathrm{MW}$ de potencia nominal y compuesta por módulos de silicio policristalino. La otra instalación solar está conectada a red desde finales del año 2008, consta de seguimiento solar en dos ejes con $1 \mathrm{MW}$ de potencia FV y los módulos empleados son de silicio monocristalino. Por su parte, la planta solar fotovoltaica localizada en la Región de Murcia presenta también una potencia nominal de 5 MW. Esta instalación está conectada a la red a través de 50 transformadores trifásicos, con un ratio de potencia de 100 kVA cada uno. La mayoría de los módulos son de capa fina conectados a 750 inversores, Sunny Mini Central 7000HV (rango de potencia de salida de $6.65 \mathrm{~kW})$.

Para la adquisición de los valores de tensión correspondientes a eventos del tipo huecos de tensión e interrupciones se ha empleado un analizador de calidad de potencia - Fluke 1760-. Se han registrado valores de tensión trifásica en el Punto de Conexión a red del lado de alta tensión de las plantas FV durante diferentes períodos enmarcados entre julio de 2008 y julio de 2013. La figura 4.3 muestra una visión general de la monitorización de la planta, así como un ejemplo de los equipos y sondas usadas durante las campañas de medidas en campo. Debido a que la tensión en el Punto de Conexión a red estaba dentro del rango de $\mathrm{kV}$ —en concreto de $20 \mathrm{kV}$ 一, y que las sondas de tensión empleadas sólo admitían un nivel máximo de tensión de $1 \mathrm{kV}$, se empleó un transformador adicional para acondicionar los datos de tensión medidos de acuerdo con las limitaciones de la sonda. A partir de las tensiones instantáneas de cada fase se han estimado los valores de tensión eficaz correspondiente. Para ello se han tenido en cuenta las definiciones de calidad de potencia de eventos de corta duración, pudiéndose encontrar más información en [11]. Atendiendo al total de campañas de medidas realizadas, se han identificado y clasificado 204 eventos distribuidos de la siguiente manera:

- 137 huecos de tensión.

- 9 interrupciones momenténeas ( $<3$ duración en segundos).

- 58 interrupciones temporales ( $\geq 3$ duración en segundos).

De acuerdo con la información facilitada por las empresas explotadoras de 


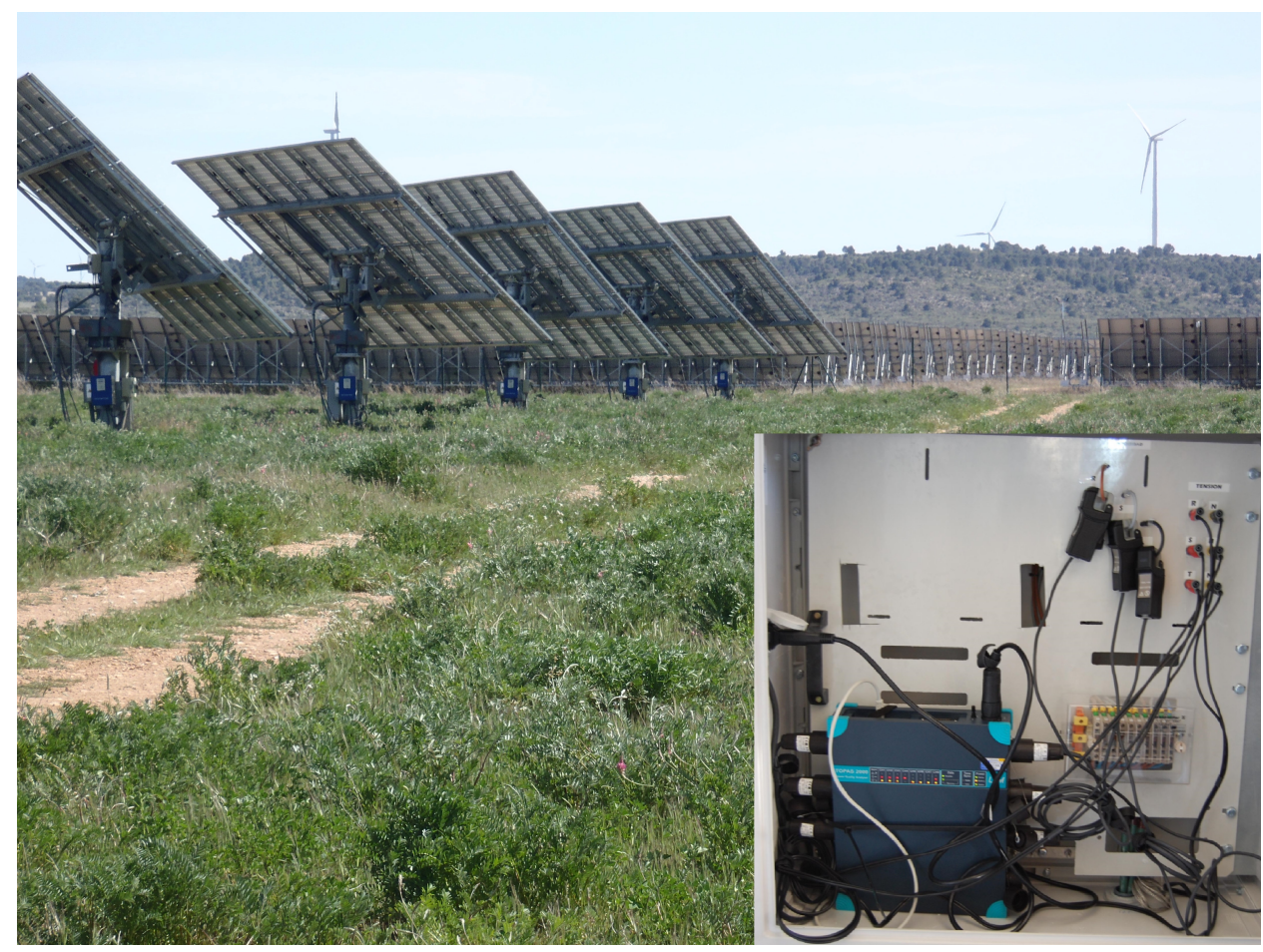

Figura 4.3: Vista general de la planta FV y el analizador de calidad de potencia-Fluke 1760-.

estas instalaciones, una gran parte de las interrupciones temporales se debieron a actividades de mantenimiento relacionadas con los módulos solares de estructura fija. Para ser exactos, estas interrupciones temporales se registraron en 2009, coincidiendo con la instalación y conexión de la planta a la red. Sin embargo, este tipo de eventos están fuera del estudio de esta tesis, centrada en la caracterización de huecos de tensión e interrupciones momentáneas y la comparación de éstos con los requerimientos de conexión a red. Por otro lado, y como un análisis preliminar de los datos registrados, hay que resaltar que el número de huecos de tensión es alrededor de 15 veces mayor que las interrupciones. Este ratio está en concordancia con contribuciones previas consultadas [90], [120], [14].

En cuanto a los registros llevados a cabos en parques eólicos, indicar que éstos se realizaron en un parque eólico situado en una zona con un perfil de velocidades de viento muy alta: Castilla-La Mancha. Dicha instalación consta de 9 aerogeneradores de $2 \mathrm{MW}$ cada uno de potencia nominal. Al igual que para la planta fotovoltaica, se ha usado un analizador de calidad de potencia comercial —Fluke 1760- Las campañas de medidas se desarrollaron de forma intermitente y a lo largo de un periodo de cinco años — desde 2006 a 


\begin{tabular}{l|c|c|c|c|c|c}
\hline Tensión Residual (pu) Duración(ms) & $\mathbf{0 - 1 0 0}$ & $\mathbf{1 0 0}-\mathbf{2 0 0}$ & $\mathbf{2 0 0}-\mathbf{4 0 0}$ & $\mathbf{4 0 0}-\mathbf{6 0 0}$ & $\mathbf{>} \mathbf{6 0 0}$ & Total \\
\hline $\mathbf{0 . 8}-\mathbf{0 . 9}$ & 36 & 29 & 5 & 4 & 7 & 81 \\
\hline $\mathbf{0 . 7}-\mathbf{0 . 8}$ & 4 & 6 & 6 & 2 & 10 & 28 \\
\hline $\mathbf{0 . 6}-\mathbf{0 . 7}$ & 2 & 2 & 0 & 0 & 3 & 7 \\
\hline $\mathbf{0 . 5}-\mathbf{0 . 6}$ & 0 & 1 & 6 & 1 & 1 & 9 \\
\hline $\mathbf{0 . 4}-\mathbf{0 . 5}$ & 0 & 1 & 0 & 0 & 3 & 4 \\
\hline $\mathbf{0 . 3} \mathbf{- 0 . 4}$ & 0 & 0 & 3 & 0 & 0 & 3 \\
\hline $\mathbf{0 . 2}-\mathbf{0 . 3}$ & 0 & 0 & 0 & 0 & 1 & 1 \\
\hline $\mathbf{0 . 1}-\mathbf{0 . 2}$ & 0 & 0 & 0 & 0 & 1 & 1 \\
\hline$<\mathbf{0 . 1}$ & 0 & 0 & 0 & 0 & 1 & 1 \\
\hline Total & 42 & 39 & 20 & 7 & 29 & 137 \\
\hline
\end{tabular}

Tabla 4.1: Resultados medidos en instalaciones FV: Tabla huecos de tensión.

2011-, registrándose un total de 303 huecos de tensión.

A partir de los eventos registrados en ambos tipos de instalaciones renovables, se abordó un análisis preliminar de los datos a través de tablas de densidad. Así, las tablas 4.1 y 4.2 caracterizan las campañas de medidas en los huertos solares y en el parque eólico respectivamente, mediante una distribución de huecos de tensión. Cada celda de la tabla muestra el número de eventos correspondiente a un rango concreto de tensión residual y de tiempo de duración global. La fila inferior resume el total de los eventos clasificados según su duración global. Como se puede ver, la mayoría de estos eventos se encuentran en la fila superior (huecos poco profundos) y en las columnas de la izquierda (tiempos de duración reducidos). De hecho, alrededor del $80 \%$ de los eventos - huecos de tensión e interrupciones - presentan un tiempo global de duración menor de 600 ms. Estos datos son ligeramente diferentes a las contribuciones previas medidas en ambientes industriales [14. En este último caso, la presencia de máquinas eléctricas grandes tiene una clara influencia en las perturbaciones, produciendo huecos de tensión más largos debido principalmente a los transitorios durante los procesos de arranque. Igualmente, la amplia mayoría de las perturbaciones medidas en las plantas FV monitorizadas están relacionadas con cortocircuitos principalmente localizados en el nivel de distribución y geográficamente cerca de estas instalaciones.

Como ya se ha comentado anteriormente, los mapas de colores suponen una representación gráfica de las tablas de densidad. En dichos diagramas se agrupan las perturbaciones por sectores rectangulares donde cada sector tiene asignado un color dependiendo del número de eventos que se registren dentro de unos rangos de tiempo total de duración y tensión residual alcanzada durante la falta. Las figuras 4.4 y 4.5 muestran el mapa de colores correspondiente a la distribución de los eventos medidos en las instalaciones fotovoltaicas y eólica. De esta figura, y en línea con las tablas 4.1 y 4.2 , los eventos recogidos presentan mayoritariamente valores de corta duración y poca profundidad. En realidad, los eventos tienen valores residuales de 


\begin{tabular}{|c|c|c|c|c|c|c|}
\hline Tensión Residual (pu) Duración (ms) & $0-100$ & $100-200$ & $200-400$ & $400-600$ & $>600$ & Total \\
\hline $0.8-0.9$ & 111 & 12 & 5 & 6 & 35 & 169 \\
\hline $0.7-0.8$ & 14 & 6 & 22 & 2 & 19 & 63 \\
\hline $0.6-0.7$ & 12 & 15 & 11 & 4 & 8 & 50 \\
\hline $0.5-0.6$ & 1 & 1 & 7 & 0 & 5 & 14 \\
\hline $0.4-0.5$ & 0 & 0 & 1 & 0 & 1 & 2 \\
\hline $0.3-0.4$ & 0 & 0 & 2 & 0 & 0 & 2 \\
\hline $0.2-0.3$ & 1 & 0 & 0 & 0 & 0 & 1 \\
\hline $0.1-0.2$ & 0 & 0 & 0 & 0 & 0 & 0 \\
\hline$<0.1$ & 2 & 0 & 0 & 0 & 0 & 2 \\
\hline Total & 141 & 34 & 48 & 12 & 68 & 303 \\
\hline
\end{tabular}

Tabla 4.2: Resultados medidos en parque eólico: Tabla huecos de tensión.

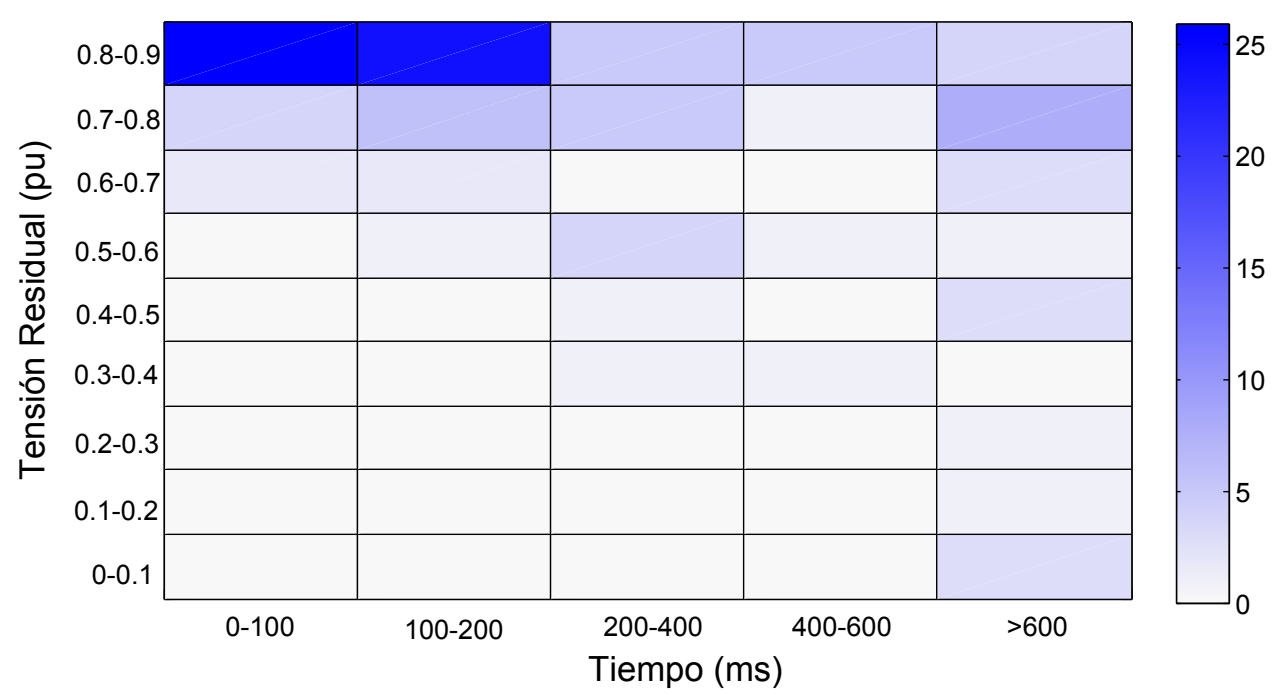

Figura 4.4: Resultados de campo en instalaciones FV: mapa de colores.

tensión entre 0,8 pu y 0., pu con una duración global menor a 100 ms.

\subsubsection{Métodos de Caracterización y Comparativa ante Requerimientos de Operación.}

A partir de todo lo comentado en los apartados anteriores, puede afirmarse que si bien existen métodos que permiten una representación simplificada de un conjunto de perturbaciones, se ha detectado sin embargo una laguna de contribuciones relativas a ofrecer soluciones visualmente ágiles para poder comparar un elevado número de perturbaciones frente requerimientos de conexión. De hecho, las tablas de densidad o los mapas de colores recogen una información limitada sobre los eventos medidos, ya que se limitan a dos términos característicos: tensión residual y tiempo de duración, lo que dificulta una comparativa eficiente frente a las normativas normalmente ex- 


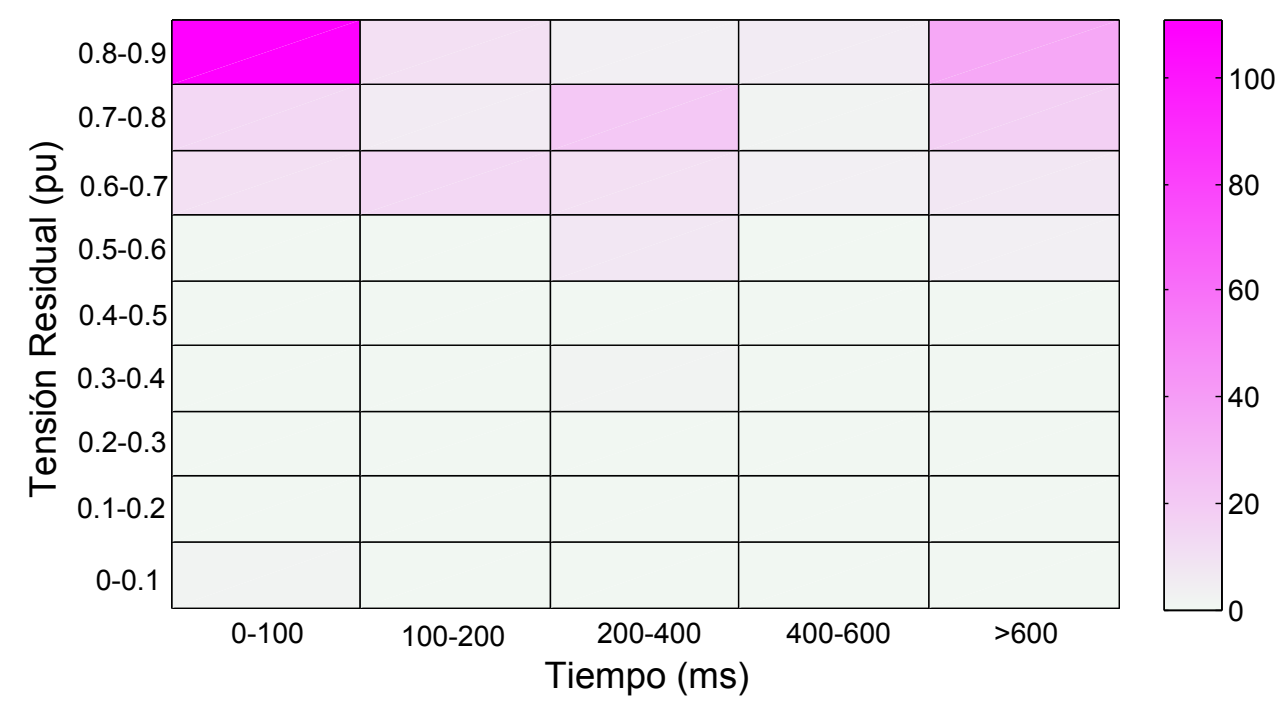

Figura 4.5: Resultados de campo en parque eólico: mapa de colores.

presadas como una limitación en la evolución de la tensión eficaz. Por tanto, se necesitarían soluciones que nos permitiesen conocer el grado de cumplimiento de los requisitos de conexión, así como proporcionar una estimación clara de cómo de severo son los eventos analizados y cuán de cerca están del limite de tensión eficaz permitido. En este sentido, recientes estudios han comprobado que además del valor de tensión eficaz residual, la severidad de las faltas es altamente dependiente de la evolución de tensión a lo largo de la perturbación y el comienzo de la onda [33]. Atendiendo a estas premisas, puede deducirse que inicialmente resultaría más apropiada una comparación basada en la superposición de trayectorias de la tensión eficaz del hueco frente al tiempo. Sin embargo, este solución presenta varias limitaciones: (i) si bien puede ser adecuada para un número reducido de eventos, resulta inoperativa si el número de eventos a analizar es elevado; (ii) no proporciona una caracterización sistemática en términos de severidad de huecos de tensión y la proximidad a los límites de tensión eficaz. Adicionalmente, y partiendo de la base de que podrían generarse un número infinito de posibles trayectorias a analizar y comparar con los límites marcados por la normativa, y que por tanto sería inviable verificar todos los posibles casos que se pueden presentar, algunos autores han propuesto recientemente técnicas computacionales con las que establecer una verificación de normativa para aeros dotados de un full-converter [121]. Estas contribuciones se basan en verificaciones para el diseño de convertidores, pero no ofrecen solución a la hora de clasificar o agrupar huecos reales.

Para superar todos estos inconvenientes, el presente capítulo describe una nueva propuesta de caracterización, clasificación y comparación de conjun- 
tos de huecos de tensión basado en el Análisis por Componentes Principales (PCA, acrónimo en inglés) y en un proceso de clustering basado en metodología $\mathrm{K}$-means. Con ello se ofrece una solución alternativa y novedosa a la identificación de distribuciones y tendencias de una gran cantidad de eventos, proponiendo patrones tipo de comportamiento de perturbaciones y permitiendo una comparativa eficiente con las limitaciones actualmente vigentes. A partir de las campañas de medidas de perturbaciones reales en parques eólicos e instalaciones fotovoltaicas comentadas anterioremente, se llevará a cabo una evaluación de la herramienta propuesta de caracterización y estimación de patrones tipo, con el fin de comparar las trayectorias de tensión eficaz promediadas con los requisitos técnicos de conexión actualmente vigente en el sistema eléctrico español, dando una estimación global de la gravedad de los eventos recogidos.

\subsection{Caracterización de Campañas de Medida: Inclusión de Severidad de Hueco.}

Tras analizar las perturbaciones medidas mediante los métodos de caracterización más comunes, se ha observado que la información que se obtiene con estos métodos no es suficiente para una correcta interpretación de la trayectoria descrita por la tensión eficaz. Así, y atendiendo a la linealización de la trayectoria del valor eficaz de tensión propuesto en el capítulo anterior, una primera aportación de este capítulo es la inclusión de un parámetro adicional con el tener en cuenta la severidad del hueco, entendido éste como el intervalo de tiempo que el hueco permanece alrededor de su valor residual. En concreto, la severidad de hueco estaría relacionada con la longitud del segmento $l_{2}$, introducido en el capítulo anterior. Por tanto, a la hora de representar un conjunto de eventos, éstas quedarían caracterizados con tres parámetros: duración, tensión residual y severidad $\left(l_{2}\right)$. Así pueden plantearse diversas opciones de representación de perturbaciones. Una primera alternativa para incluir la severidad de las perturbaciones viene representada en la figura 4.6, donde se muestran los huecos de tensión correspondientes a la instalación solar fotovoltaica. Debido al número elevado de perturbaciones, se ha hecho una subdivisión de acuerdo con el tiempo de duración total del hueco de tensión, para clarificar así la distribución de eventos. De esta forma es más fácil representar los huecos de tensión en términos no sólo de su valor de tensión residual y tiempo de duración del hueco, sino que también se considera el tiempo de permanencia alrededor del valor mínimo de tensión $\left(\forall V V \in\left[V_{\min }, 1,1 \cdot V_{\min }\right]\right)$. Consecuentemente, las esferas tridimensionales con mayor radio serán las que presenten mayor tiempo alrededor del valor de la tensión residual. Al incluir la severidad de hueco como el tiempo de permanencia alrededor del mínimo, sería posible detectar como huecos ciertamente críticos aquéllos que, aún teniendo una tensión residual alta (por 


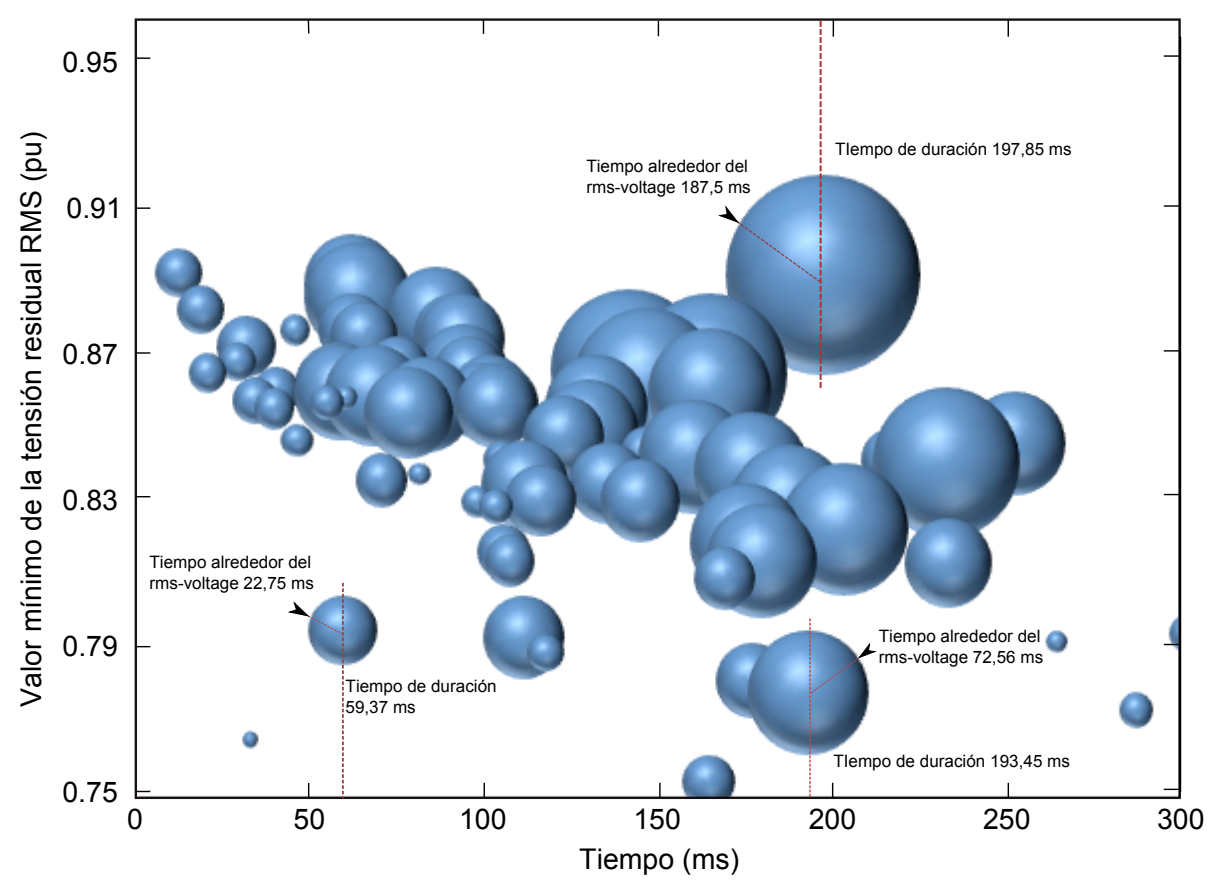

Figura 4.6: Resultados medidos en la planta FV: Propuesta de diagrama de dispersión para evaluar la severidad de hueco de tensión.

ejemplo $0,75 \mathrm{pu}$ ) permanecen casi todo el tiempo del hueco alrededor de ese valor. Por lo tanto, si el hueco presenta una gran parte de la duración del mismo alrededor de esa tensión residual, éste podría ser más severo que un hueco con menor tensión residual pero que se recuperase muy rápidamente. Esta clase de representación permite comparar la severidad de las perturbaciones frente a los intervalos de tiempo y valores máximos de tensión residual que demandan los requisitos técnicos actuales de conexión. En efecto, estos requisitos especifican claramente el intervalo de tiempo en torno a las tensiones mínimas de los recursos energéticos renovables en general y para las centrales fotovoltaicas conectadas a la red, en particular, como se representa en la figura 4.7 .

La mayoría de los eventos reales presentan un valor de tensión mínima pero, por lo general, no todos los eventos se mantienen en este mínimo durante todo el tiempo de duración del hueco, por eso se propone un intervalo de tensión alrededor del valor mínimo correspondiente para ser capaces de definir esta característica. De esta manera, y tal y como se ha comentado en el párrafo anterior, se asume hasta un $10 \%$ por encima de la tensión residual correspondiente como intervalo de tiempo que caracteriza la gravedad de la perturbación y el tiempo alrededor de la tensión mínima - recuérdese la definición de $l_{2}$ del capítulo anterior-. Teniendo en cuenta esta suposi- 


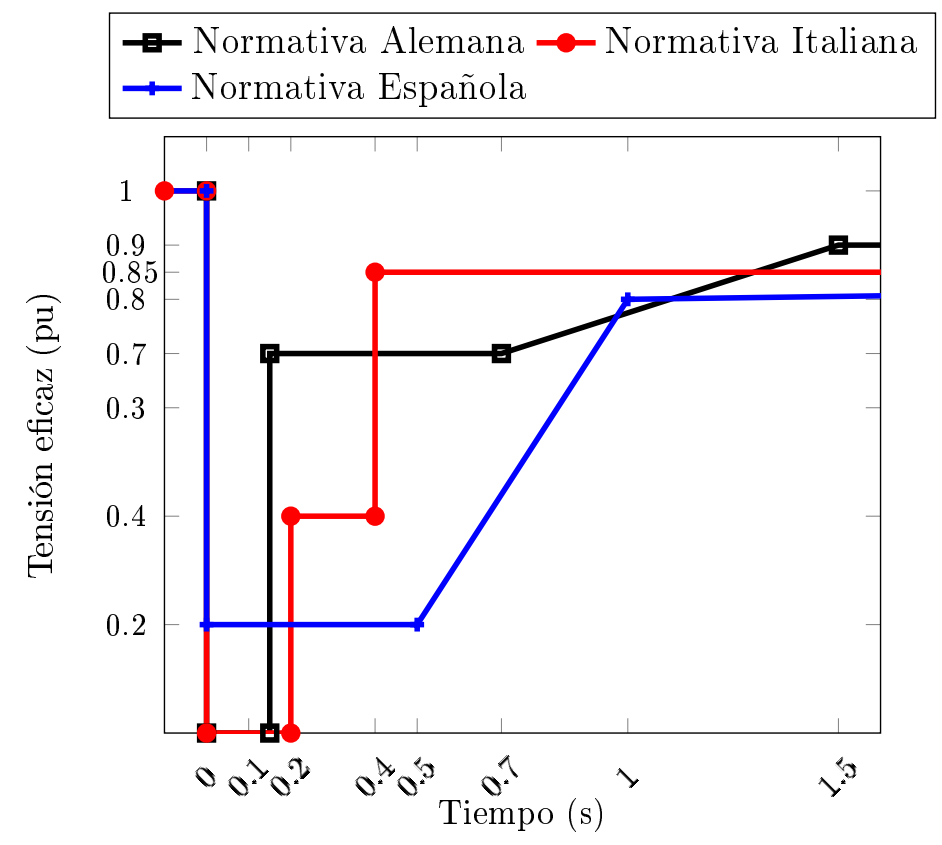

Figura 4.7: Comparativa de los requerimientos para plantas FV en el punto de conexión con la red (Ejemplos de normativa europea)

ción, la figura 4.8 compara los requerimientos de conexión alemán, español e italiano con los eventos medidos. Los huecos de tensión se representan por la definición dada del dato mínimo de tensión, así como su intervalo alrededor de estos valores mínimos de tensión. De acuerdo con los requerimientos de conexión, las centrales FV deben soportar perturbaciones por encima de los valores de tensión residuales mínimos sin desconexiones. Por otra parte, las instalaciones FV para los requerimientos de conexión alemanes e italianos deben soportar los eventos con $100 \%$ del hueco de tensión — valor de tensión residual cero-.

Si la valoración de la comparativa se lleva a cabo considerando tanto los huecos medidos como los requerimientos de conexión, las plantas FV tienen que soportar sin desconectarse la mayoría de los huecos de tensión. Los requerimientos italianos son los más estrictos comparados con el caso alemán, en términos de intervalo de tiempo alrededor de la tensión residual —200 ms- Sin embargo, la cantidad de eventos registrados que tienen que soportar las instalaciones FV son bastante similares al caso alemán (89\%). En relación con los valores de tensión residual, el requerimiento de conexión español es menos severo en comparación con los otros dos países, aunque es más exigente en términos de intervalo de tiempo máximo en torno a la tensión mínima - $500 \mathrm{~ms}$ - . De esta manera, y debido a que la mayoría de las perturbaciones registradas presentan una alta tensión residual y consecuentemente baja profundidad, las instalaciones FV tienen que soportar alrededor 


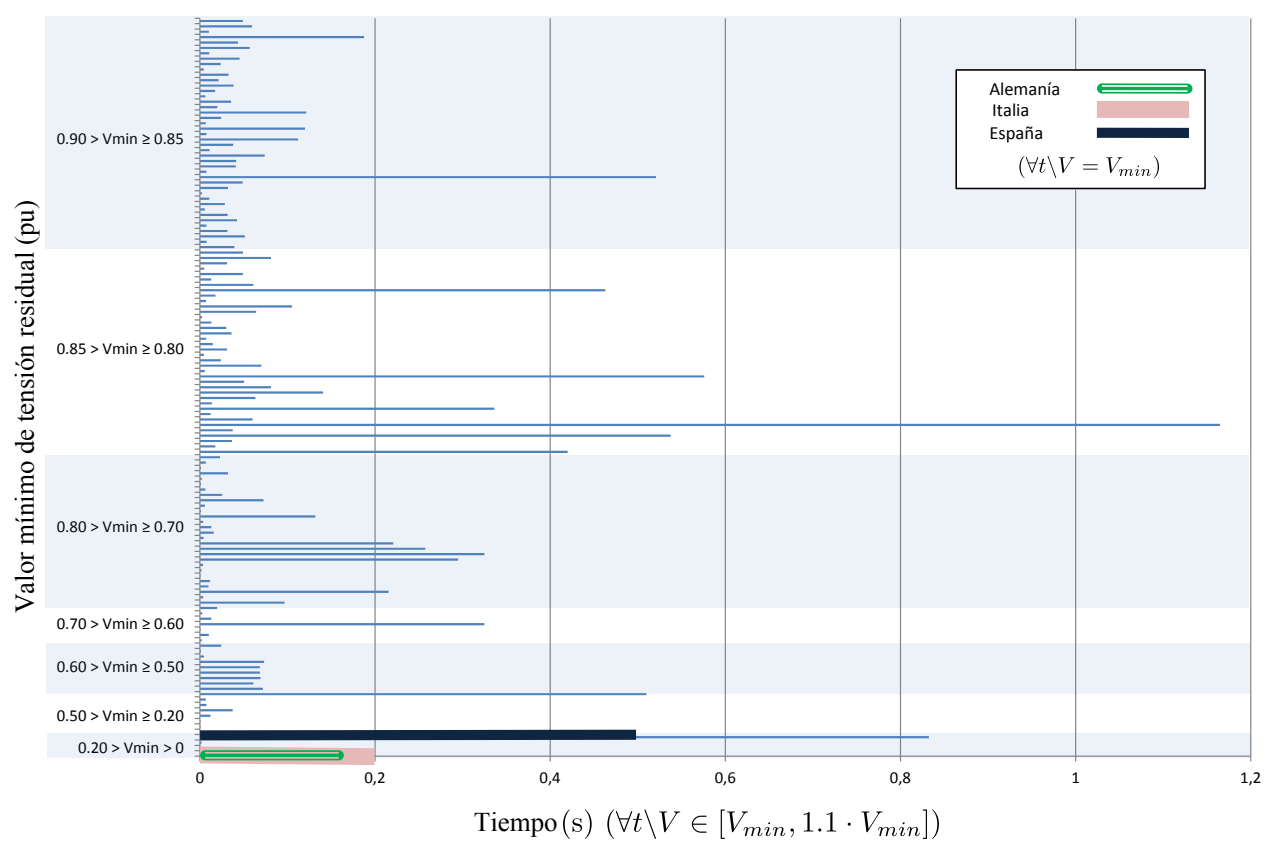

Figura 4.8: Resultados medidos en la planta FV: Comparativa con los requisitos de conexión.

del $92 \%$ de los eventos sin desconectarse. Por lo tanto, y como conclusión de esta comparativa, los requisitos de conexionado europeo que incluyen requerimientos específicos para plantas FV proporcionan actuaciones graves y altamente exigentes para este tipo de instalaciones, y deben soportar la mayoría de los eventos sin desconexiones. Estas características deberían ser revisadas y en su mayoría incluidas en el rendimiento del inversor, ya que son componentes muy sensibles de las instalaciones fotovoltaicas bajo huecos de tensión e interrupciones.

Esta forma de caracterización y representación de huecos de tensión ofrece más información que los métodos de caracterización discutidos anteriormente. Sin embargo, tal y como se ha visto, no ofrece una solución práctica y plausible para caracterizar grandes conjuntos de perturbaciones y poder compararlas con requerimientos actuales de conexión. Téngase en cuenta que el mero análisis de los eventos correspondientes a las instalaciones FV, muchos menores en número que los obtenidos en el parque eólico, ya dificultan su lectura y producen un solapamiento de unos con respecto a otros. Por tanto, para una correcta interpretación de la caracterización de grandes grupos de perturbaciones sería necesario proponer metodologías alternativas de visualización y representación. Este objectivo es el que se aborda en el siguiente apartado. 


\subsection{Metodología Propuesta: Caracterización mediante Componentes Principales y Agrupación basada en K-means.}

En relación con lo expuesto en el apartado anterior, uno de los problemas que se plantean a la hora de analizar un conjunto de eventos es que la representación conjunta de un número elevado de perturbaciones entorpece su caracterización y dificulta la visualización agregada de los mismos. Así pues, resulta complicada una comparativa directa entre los requerimientos de conexión y dichas perturbaciones reales, en términos de severidad y de proximidad (o no) a los límites impuestos por las normativas actuales. Para solucionar este problema podrían proponerse diferentes opciones con las que representar un conjunto de eventos. La solución más aceptada en anteriores contribuciones pasa por reducir el número de variables a representar, con la consecuente pérdida de información de cada evento, aspecto ya comentado en diversos apartados de esta tesis. Como método alternativo para la caracterización de perturbaciones, se propone en este capítulo un análisis estadístico a través de componentes principales y de forma paralela un agrupamiento de eventos con características similares mediante un proceso de clustering basado en técnicas de $\mathrm{K}$-means. Con ello se ofrece una solución no basada en la reducción de variables, sino en la agrupación de eventos con similares características. A partir de estas agrupaciones pueden deducirse patrones de comportamiento de eventos más probables, limitando el número de trayectorias de tensión eficaz a comparar con los requerimientos de operación.

\subsubsection{Análisis por Componentes Principales. Descripción General.}

Con el objetivo de caracterizar los huecos de tensión con la mayor precisión posible, en la sección 3.4 del capítulo 3 se describen un conjunto de segmentos como propuesta alternativa para la caracterización de la evolución de la tensión eficaz a lo largo de una perturbación. Este proceso de linealización, si bien identifica de forma clara los eventos, introduce un mayor número de variables para cada perturbación. De hecho, se cuenta con cuatro valores de longitudes de segmento por evento caracterizado. Así pues, cuando se pretenden analizar una gran cantidad de huecos de tensión, este número de variables se presenta inicialmente elevado; siendo deseable alcanzar un término medio entre precisión y simplicidad que minimice, al mismo tiempo, el número de variables a manejar. Por otro lado, la solución aportada debería permitir una visualización ágil y clara de los eventos analizados; permitiendo realizar comparativas con las trayetorias límites impuestas por los requerimientos de conexión actuales. Teniendo en cuenta estas premisas, se propone como paso inicial una reducción del número de variables asociadas a cada 


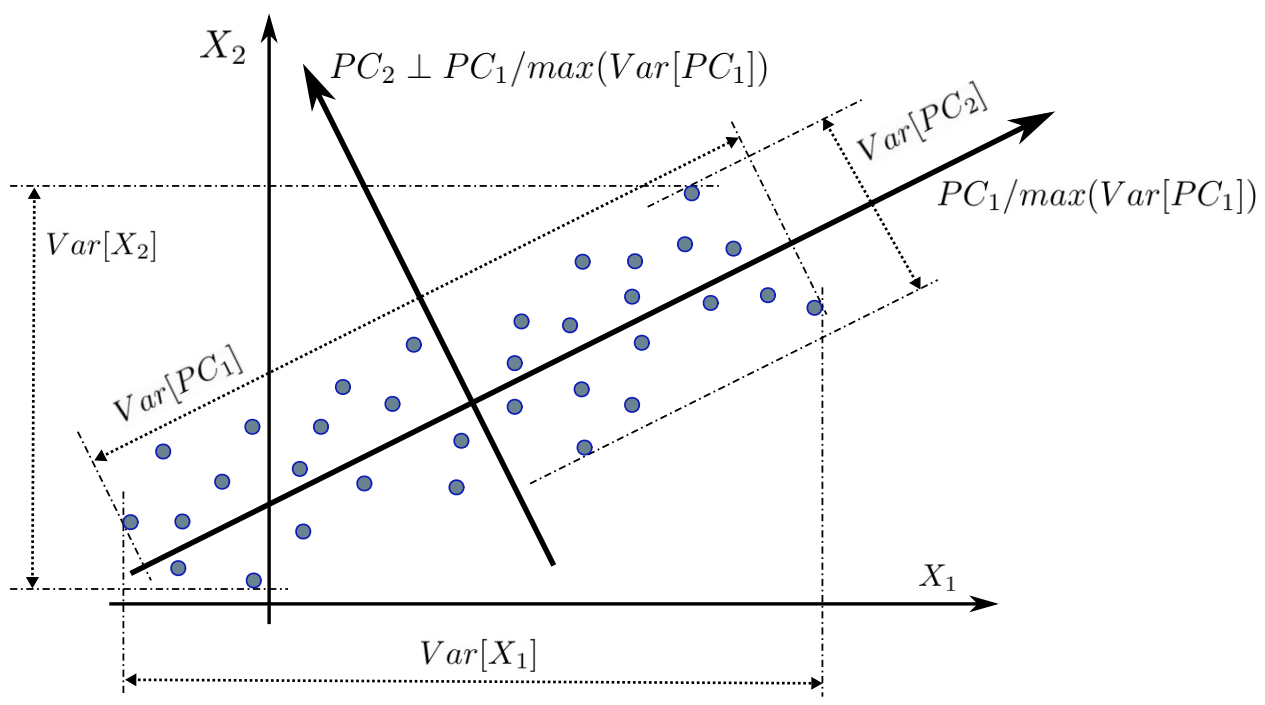

Figura 4.9: Análisis de componentes. Resumen general.

evento mediante el Análisis por Componentes Principales (ACP), para más información puede consultarse 122 Capítulo 14. Esta es una técnica estadística en la que, a partir de un conjunto datos identificados por las variables $X_{1}, X_{2}, \ldots, X_{p}$, propone una reducción de la dimensión de estas variables sin pérdida dramática de la información. Es decir, se propone un nuevo conjunto de variables, combinación lineal de las primeras y denominadas componentes principales, en las que se conserva la variabilidad del conjunto inicial. Expresado en términos matemáticos, las $p$ componentes principales derivadas del conjunto inicial tendrían la siguiente estructura:

$$
\begin{aligned}
P C_{1} & =\lambda_{11} X_{1}+\lambda_{12} X_{2}+\ldots+\lambda_{1 p} X_{p} \\
P C_{2} & =\lambda_{21} X_{1}+\lambda_{22} X_{2}+\ldots+\lambda_{2 p} X_{p} \\
\vdots & =\vdots \\
P C_{p} & =\lambda_{p 1} X_{1}+\lambda_{p 2} X_{2}+\ldots+\lambda_{p p} X_{p} .
\end{aligned}
$$

Las combinaciones lineales anteriores se normalizan, cumpliéndose para cada columna $i$, la siguiente relación: $\lambda_{i 1}^{2}+\lambda_{i 2}^{2}+\ldots+\lambda_{i p}^{2}=1$.

Una característica crucial a la hora de seleccionar las componentes principales es que ha de maximizarse su variabilidad. De esta forma, y comenzando por la componente principal $P C_{1}$, se seleccionará aquélla para la cual su variabilidad es máxima; es decir, no existe otra combinación lineal normalizada de las variables $X_{1}, X_{2}, \ldots, X_{p}$ que presente una variabilidad mayor que $P C_{1}$. Además, ha de cumplirse que $P C_{1}, P C_{2} \ldots P C_{p}$ no estén correlacionadas: $\operatorname{corr}\left(P C_{i}, P C_{j}\right)=0$ para todo $i$ y $j$. Por tanto, y continuando con nuestra deducción, $P C_{2}$ presenta la mayor variabilidad entre todas las 
combinaciones lineales normalizadas de las variables originales que no estén correlacionadas con $P C_{1}$. De manera similar, $P C_{3}$ presenta la mayor variabilidad entre todas las combinaciones lineales normalizadas que no están correlacionadas con $P C_{1}$ y $P C_{2}$; y así sucesivamente. Además, y tal y como se ha comentado al comienzo del párrafo, se conserva la variabilidad total del conjunto de valores inicial:

$\operatorname{var}\left(X_{1}\right)+\operatorname{var}\left(X_{2}\right)+\ldots+\operatorname{var}\left(X_{p}\right)=\operatorname{var}\left(P C_{1}\right)+\operatorname{var}\left(P C_{2}\right)+\ldots+\operatorname{var}\left(P C_{p}\right)$.

No obstante, y debido a la metodología de selección de las componentes principales, sí es cierto que se cumple:

$$
\operatorname{var}\left(P C_{1}\right) \geq \operatorname{var}\left(P C_{2}\right) \geq \ldots \geq \operatorname{var}\left(P C_{p}\right) .
$$

Esta última característica es clave para conseguir una reducción de las dimensiones de las variables iniciales. Así, si las primeras componentes principales abarcan una elevada variabilidad es posible entonces considerar sólo dichas componentes, despreciando el resto y facilitando su análisis e incluso su representación grafica. De esta forma sería posible alcanzar una reducción de las dimensiones iniciales, considerando sólo las primeras componentes como una representación aproximada del conjunto de datos inicial. Esto puede ser particularmente útil para casos en los que el número de variables iniciales $\left(X_{1}, X_{2}, \ldots X_{p}\right)$ es mayor de tres, y por tanto no se puede realizar una representación gráfica de las mismas. De este modo, y dado que la primera componente $P C_{1}$ muestra la mayor variabilidad de todas las combinaciones posibles, el máximo de reducción de componentes sería sólo considerar $P C_{1}$, con la consecuente pérdida de información — en términos de variabilidaddel conjunto inicial. Por otro lado, y a través del valor de los pesos $\left(\lambda_{i j}\right)$, puede determinarse la aportación de cada variable $\left(X_{j}\right)$ a la componente principal, identificando aquellas variables originales que más contribuyen a $P C_{1}$. Este último análisis es aplicable a cualquier componente principal, a partir de los valores de $\operatorname{los} \lambda_{i j}$ correspondientes.

Con el fin de clarificar las ideas aquí expuestas en cuanto a aplicación de componentes principales, la figura 4.9 muestra un ejemplo de un conjunto inicial de datos bi-dimensionales caracterizados por las variables $X_{1}$ y $X_{2}$. La aplicación del análisis por componentes principales supondría la estimación de $P C_{1}$ y $P C_{2}$, variables no correlacionadas y que mantienen los requerimientos previos. Además, en este caso se cumple que $\operatorname{var}\left(P C_{1}\right)>>\operatorname{var}\left(P C_{2}\right)$, con lo que en consecuencia el conjunto inicial de datos bi-dimensionales podría reducirse y caracterizarse por sólo por una variable unidimensional a través de $P C_{1}$. Esta transformación y la reducción de componentes implicaría la pérdida de información respecto a los datos originales. En concreto, se desestimaría la variabilidad que posee $P C_{2}$ y se supondría que el conjunto inicial está suficiente representado por la componente $P C_{1}$. Una reducción 
en las dimensiones iniciales resulta es muy útil, tal y como se ha comentando anteriormente, ya que en ocasiones permite una representación gráfica o bien una reducción de las dimensiones de dicha representación. Por ejemplo, en el caso de la figura 4.9 pasaríamos de una representación bi-dimensional mediante $X_{1}$ y $X_{2}$ a una uni-dimensional basadá sólo en $P C_{1}$.

Matemáticamente, las componentes principales se obtienen como los vectores propios de la matriz de covarianza del conjunto de datos, para más información consultar la referencia [122]. En el caso que nos ocupa, y a partir del conjunto de segmentos definido en el apartado sección 3.4 , se tendría que $p=4$ y que el conjunto inicial de variables $\left(X_{1}, X_{2}, \ldots, X_{p}\right)$ estaría representado por las longitudes $\left(l_{1}, l_{2}, l_{3}, l_{4}\right)$. Así pues, partiríamos de conjuntos de eventos caracterizados mediante cuatro valores, los cuales suponen la linealización por tramos de la trayectoria de la tensión eficaz de la fase más afectada por la perturbación. Para cada conjunto de eventos, se propone como metodología de reducción de variables la aplicación del análisis por componentes principales, escogiendo si es posible un número más reducido de componentes que permita la representación gráfica y la visualización de los eventos. Ejemplos prácticos de esta técnica estadística se encuentran recogidos en el apartado 4.4 de este capítulo.

\subsubsection{Agrupación basada en K-means. Identificación de Patrones.}

En paralelo al cálculo de componentes principales y la posible reducción de variables con las que caracterizar cada evento, se propone en este apartado una solución para poder visualizar un conjunto de perturbaciones y comparar con los requirimientos de operación. El objetivo se centra en identificar grupos de eventos que posean caracterícticas similares, y estimar patrones-tipo con los que reducir el número de trayectorias a comparar con las actuales normativas. Con tal fin, se propone un proceso basado en $\mathrm{K}$ means clustering a partir de los parámetros $l_{1}, l_{2}, l_{3}, l_{4}$. Así pues, se trata de identificar comportamientos similares en base a las longitudes de estos segmentos, proponiendo perfiles-tipo en los que queda representada la tensión residual del hueco, el tiempo transcurrido alrededor de este valor mínimo y las pendientes de entrada al hueco y recuperación. Este tipo de caracterización y agrupación de eventos supone una de las contribuciones relevantes de la presente tesis, no habiéndose encontrado en la literatura específica aportaciones similares al problema del análisis e identificación de conjuntos de perturbaciones. Además, los perfiles representativos de tensión eficaz se pueden comparar de manera ágil y directa con los requerimientos de conexión actuales, dando un estimación media de la severidad de las perturbaciones en términos de los límites de tensión eficaz requeridos por los procedimientos de operación.

En cuanto a la metodologia escogida basada en K-means clustering, ésta 
se basa en la identificación de clusters y centros de clusters en un conjunto de datos. El usuario necesita especificar el número de centros de cluster y la estimación inicial para ellos, y el procedimiento K-means desplaza de forma iterativa los centros para minimizar la varianza total del cluster. Concretamente, dado un conjunto inicial de centros, el algoritmo K-means engloba dos etapas:

- Cada punto es asignado a su centro más cercano

- Se calculan las medias de cada variable para los puntos de datos en cada cluster, y este vector medio se convierte en el nuevo centro de este cluster.

Estos dos pasos se iteran hasta que las diferencias son menores de un umbral escogido, llegando así a la convergencia del proceso. Normalmente los centros iniciales son observaciones elegidas aleatoriamente de los datos de partida, y supone un punto a tener en cuenta por el usuario de estas metodologías. Para más información, consultar la referencia [122], página 460.

A partir de todo lo expuesto en este apartado 4.3.2 y en el anterior (ver apartado 4.3.1 , la figura 4.10 sintetiza la metodología propuesta para caracterizar y representar un grupo de perturbaciones. Primeramente se describe cada hueco de tensión a través del conjunto de longitudes $\left(l_{1}, l_{2}, l_{3}, l_{4}\right)$ que contienen información de la evolución del evento. Posteriormente se aplica el Análisis de Componentes Principales (ACP) para reducir la dimensión del conjunto de datos original, manteniendo aquel número de componentes que explican con suficiente nivel de exactitud la variabilidad de los datos. Para minimizar la pérdida de información como consecuencia de tomar un conjunto reducido de componentes principales, se ha seleccionado un número tal que abarque al menos el $90 \%$ de viabilidad inicial. Por otro lado, y de forma paralela, se aplica un proceso de agrupación o clustering basado en K-means para identificar los patrones y similitudes en el conjunto de eventos. Finalmente, y considerando las salidas de ambos procesos, se tiene un conjunto promedio de perfiles de tensión que representan los eventos más comunes. Estos perfiles pueden ser fácilmente comparables con los requisitos de procedimientos de operación actuales, todos ellos basados en trayectorias de tensión eficaz para la fase más afectada por el hueco. 


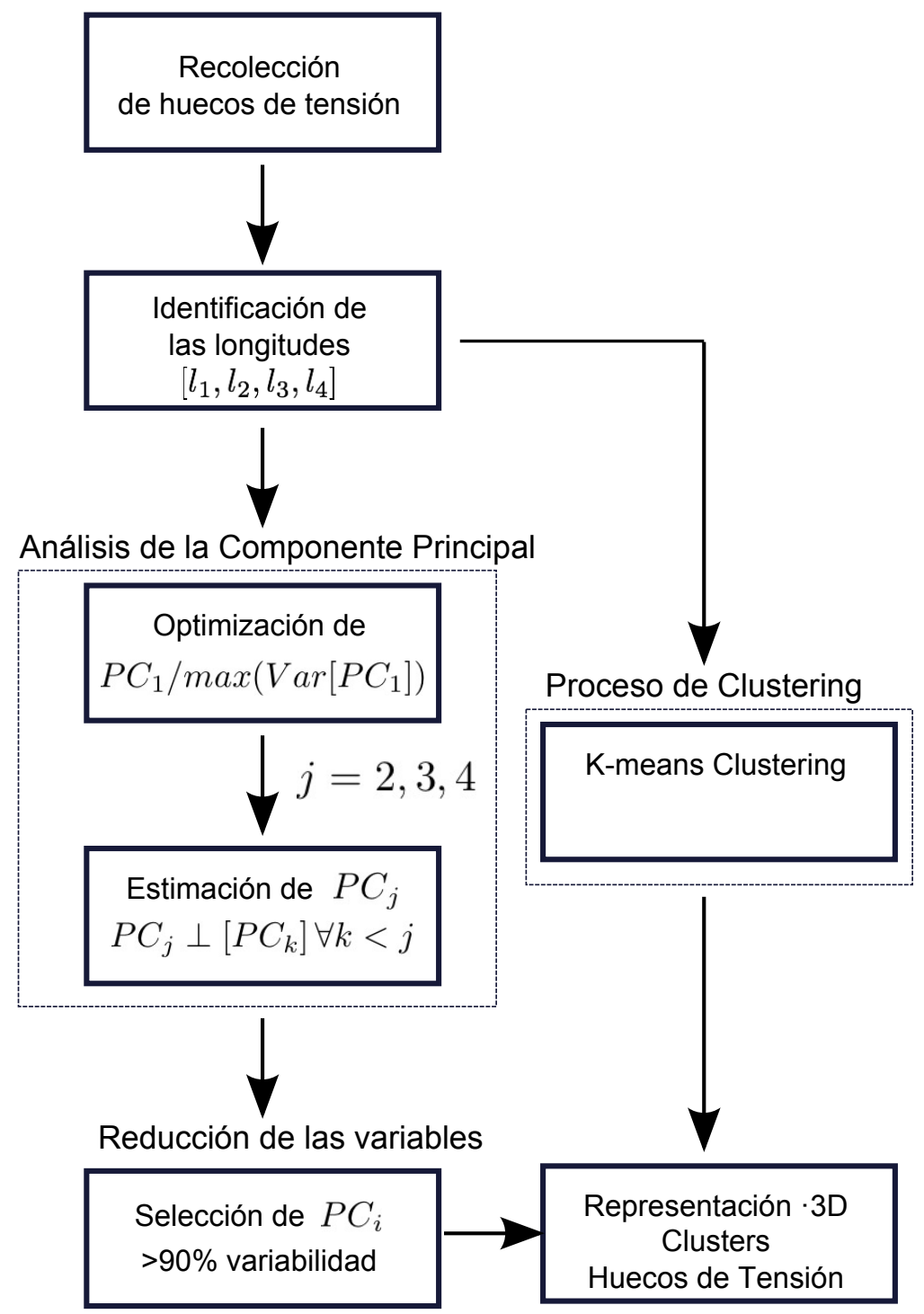

Figura 4.10: Análisis de Componente Principal y K-means Clustering. Descripción General. 


\begin{tabular}{ccccc}
\hline & $P_{1}$ & $P_{2}$ & $P C_{3}$ & $P C_{4}$ \\
\hline Desviación estándar & 0,27 & 0,19 & 0,15 & 0,05 \\
Proporción de variabilidad & 0,54 & 0,28 & 0,16 & 0,02 \\
Proporción acumulada & 0,54 & 0,82 & 0,98 & 1 \\
\hline
\end{tabular}

Tabla 4.3: Huecos de tensión en parque eólico. Distribución de variabilidad por componentes principales.

\subsection{Resultados: Aplicación a Campañas de Medida Reales y Comparativa con Requerimientos de Operación.}

Considerando la metodología expuesta en el apartado anterior (ver 4.3), se ha llevado a cabo una extensa evaluación de esta propuesta tomando como eventos los medidos en instalaciones fotovoltaicas y eólicas que se describieron en el apartado 4.1.2 Para todos ellos se ha realizado primeramente una etapa de identificación del conjunto de longitudes, caracterizando así la tensión eficaz correspondiente a la fase más efectada por la perturbación. A partir de estas longitudes $\left(l_{1}, l_{2}, l_{3}, l_{4}\right)$, estimadas para cada perturbación, se ha aplicado un análisis por componentes principales para reducir la dimensión inicial de las longitudes. En paralelo se ha realizado un proceso de clustering con el que identificar patrones-tipo más comunes y poder así hacer una comparativa con requerimientos de conexión actualmente aplicables en el sistema eléctrico español. Todo ello, tal y como se discutió anteriormente, queda resumido a modo de esquema en la figura 4.10 .

\subsubsection{Aplicación ACP y K-means a campañas de medida en parque eólico.}

Partiendo del conjunto de eventos resumido en la Tabla 4.2, y teniendo en cuenta el cálculo de longitudes $\left(l_{1}, l_{2}, l_{3}, l_{4}\right)$ para cada una de estas perturbaciones, el análisis por componentes principales queda resumido en la tabla 4.3. Tal y como puede apreciarse, y de acuerdo con los resultados derivados de este análisis estadístico, la primera componente $P C_{1}$ incluiría el $54 \%$ del total de la variabilidad de eventos, mientras que la segunda componente $P C_{2}$ englobaría aproximadamente el $28 \%$, dejando el tercer componente restante $P C_{3}$ con la explicación del $16 \%$ de la variabilidad, y una aportación casi marginal añadiría la componente $P C_{4}$. Así, pues se podría inicialmente reducir la dimensión del conjunto inicial de cuatro a dos parámetros, tomando como valores representativos $P C_{1}$ y $P C_{2}$, y englobando así más del $80 \%$ de la variabilidad del conjunto inicial de eventos.

En la tabla 4.4 se muestran los valores de $\lambda_{i j}$ correspondientes a las primeras componentes principales $\left(P C_{1}, P C_{2}, P C_{3}\right)$. Tal y como se ha comentado 


\begin{tabular}{ccccc}
\hline & $l_{1}$ & $l_{2}$ & $l_{3}$ & $l_{4}$ \\
\hline$P C_{1}$ & 0,09 & $-0,99$ & 0,12 & $-0,03$ \\
$P C_{2}$ & 0,67 & 0,14 & 0,71 & 0,16 \\
$P C_{3}$ & $-0,06$ & $-0,05$ & $-0,17$ & 0,98 \\
\hline
\end{tabular}

Tabla 4.4: Huecos de tensión en parque eólico. Coeficientes por componentes principales.

anteriormente, se propone una reducción efectiva efectiva y conveniente de la dimensión de parámetros iniciales, teniendo en cuenta sólo las dos primeras componentes del algoritmo ACP, quedando así una disminución de las cuatro longitudes iniciales en dos componentes principales con una pérdida variabilidad menor al $20 \%$. Las expresiones que relacionan las dos primeros componentes principales serían pues:

$$
\begin{aligned}
& P C_{1}, \text { Wind }=-0,09 l_{1}+0,99 l_{2}-0,12 l_{3}+0,03 l_{4} \\
& P C_{2}, \text { Wind }=0,67 l_{1}+0,14 l_{2}+0,71 l_{3}+0,16 l_{4} .
\end{aligned}
$$

La primera componente está dominada por $l_{2}$, el intervalo de tiempo correspondiente a la evolución de la tensión eficaz alrededor del valor de la tensión residual. La segunda componente posee una mayor presencia de $l_{3}$ y de $l_{1}$, dos características que básicamente relatan cómo es el proceso de entrada de hueco y de despeje del mismo. Perturbaciones con altos valores de $P C_{2}$, por lo general, implicarían tensiones eficaces residuales bajas y por lo tanto, importante reducción de la tensión eficaz.

En concordancia con el esquema representado en la figura 4.10, se propone una visualización bi-dimensional de $P C_{1}$ y $P C_{2}$ como una representación reducida del conjunto inicial de parámetros. Esta es la primera ventaja de esta solución, reducir la dimensión del conjunto inicial de parámetros (longitudes) y permitir una representación visual de los eventos recogidos. En este caso, reducir la caracterización supone representar más del $80 \%$ de la variabilidad del grupo inicial de perturbaciones, mientras se disminuye en la mitad el número de variables a considerar (de cuatro longitudes a sólo dos componentes principales). Así, los 293 huecos de tensión recogidos en las campañas de medición en campo se representan en la figura 4.11 mediante sus parámetros $P C_{1}$ y $P C_{2}$. Esta es una proyección bi-dimensional de las componentes principales reducidas sólo a sus dos primeras direcciones principales: $P C_{1}$ y $P C_{2}$. Cada hueco individual está etiquetado con su número de cluster, resultante de aplicar la etapa de K-means clustering al conjunto de eventos. Es de destacar que la mayoría de los eventos se distribuyen a lo largo de dos líneas, excepto básicamente el grupo número 11, que está situado en otra zona ajena a estas dos líneas. La línea formada por los grupos 3,8 y 6 están dominados por un incremento progresivo de $P C_{1}$, asociado principalmente al intervalo de tiempo correspondiente a la evolución de la 


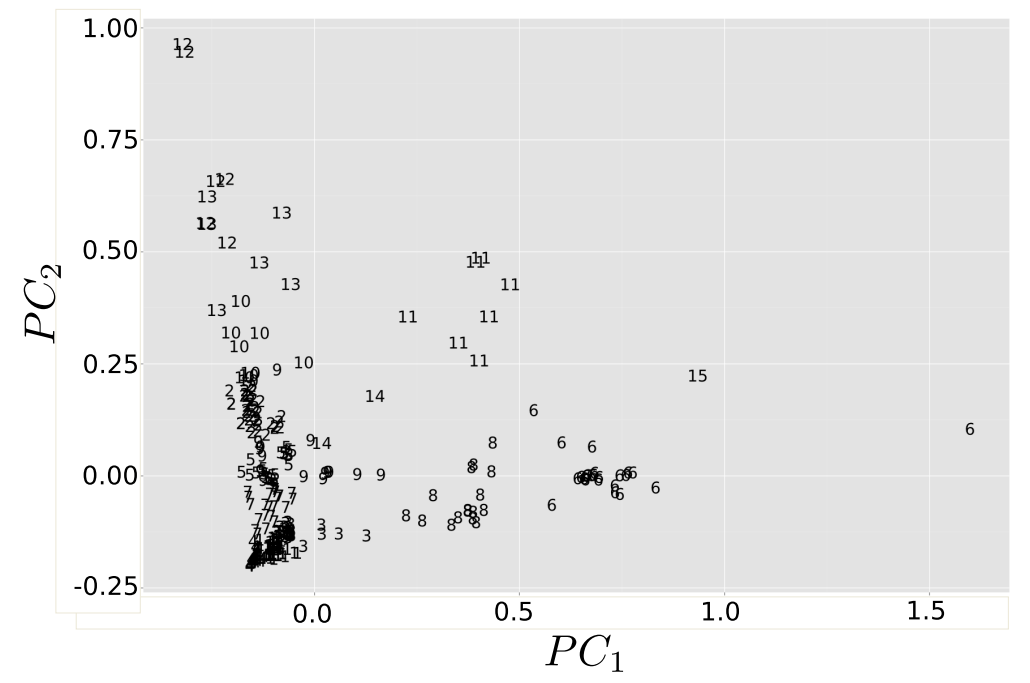

Figura 4.11: Huecos de tensión en parque eólico. Caracterización por componentes $P C_{1}$ y $P C_{2}$. Identificación de grupos y patrones mediante $\mathrm{K}$-means Clustering.

tensión eficaz en torno al valor residual $\left(l_{2}\right)$, y una disminución de $P C_{2}$, relacionada básicamente con la profundidad del hueco. En cuanto a la línea donde podrían englobarse los grupos de 7, 9, 10, 13 y 12, se tiene un incremento de $P C_{2}$ mientras que $P C_{1}$ permanece casi invariable y con un valor reducido, lo que corresponde a huecos más profundos y de poca duración. Esta interpretación de los datos se confirma con la tabla 4.5 que muestra respectivamente la media y desviación estándar de cada longitud (desde $l_{1}$ a $l_{4}$ ) dentro de los 15 clusters. La definición de las longitudes se han discutido en el capítulo anterior, ver la figura 3.20 . Además, la tabla 4.6 resume los clusters, en términos de media y desviación típica para los principales parámetros relacionados con los valores de tensión y duración. Estos parámetros están en línea con las características pre-definidas que tienen que ser identificadas en cada hueco: el valor mínimo de la tensión eficaz $\left(V_{\min }\right)$, el tiempo global de duración del evento $(\Delta t)$, el intervalo de tiempo alrededor de la tensión eficaz mínima $\left(\Delta t\left(V_{\min }\right)\right)$ y el intervalo de tiempo parcial tomado para recuperar hasta $0,8 \mathrm{pu}\left(\Delta t_{0,8}\right)$.

A partir de la representación de los eventos y su etiquetado en función del grupo al que pertenecen, se propone una manera adicional de representarlos en función del número de huecos presentes. Así, la figura 4.12 representa un mapa de calor de la densidad de presencia de valores $P C_{1}$ y $P C_{2}$ para los huecos en estudio. Esta representación gráfica complementa la presentada en la figura 4.11, y viene a destacar las zonas con una mayor presencia de eventos y, por tanto, los valores más habituales tanto de $P C_{1}$ como de $P C_{2}$ para los huecos medidos. 


\begin{tabular}{cccccccccc}
\hline Cluster & avg $\left(l_{1}\right)$ & $\sigma\left(l_{1}\right)$ & avg $\left(l_{2}\right)$ & $\sigma\left(l_{2}\right)$ & avg $\left(l_{3}\right)$ & $\sigma\left(l_{3}\right)$ & $\operatorname{avg}\left(l_{4}\right)$ & $\sigma\left(l_{4}\right)$ & Número de huecos \\
\hline 1 & 0.03 & 0.02 & 0.07 & 0.02 & 0.00 & 0.00 & 0.03 & 0.02 & 48 \\
2 & 0.28 & 0.02 & 0.05 & 0.03 & 0.19 & 0.03 & 0.10 & 0.00 & 43 \\
3 & 0.07 & 0.02 & 0.11 & 0.04 & 0.00 & 0.00 & 0.07 & 0.02 & 37 \\
4 & 0.02 & 0.02 & 0.01 & 0.01 & 0.00 & 0.00 & 0.02 & 0.02 & 31 \\
5 & 0.19 & 0.02 & 0.08 & 0.04 & 0.09 & 0.02 & 0.10 & 0.01 & 26 \\
6 & 0.09 & 0.04 & 0.90 & 0.20 & 0.01 & 0.03 & 0.10 & 0.01 & 24 \\
7 & 0.13 & 0.02 & 0.06 & 0.03 & 0.03 & 0.03 & 0.10 & 0.01 & 22 \\
8 & 0.07 & 0.05 & 0.53 & 0.06 & 0.01 & 0.02 & 0.07 & 0.04 & 16 \\
9 & 0.13 & 0.04 & 0.12 & 0.10 & 0.03 & 0.05 & 0.66 & 0.13 & 15 \\
10 & 0.37 & 0.04 & 0.07 & 0.05 & 0.28 & 0.05 & 0.10 & 0.01 & 9 \\
11 & 0.37 & 0.08 & 0.62 & 0.08 & 0.28 & 0.05 & 0.10 & 0.00 & 7 \\
12 & 0.70 & 0.14 & 0.03 & 0.03 & 0.60 & 0.14 & 0.10 & 0.00 & 6 \\
13 & 0.32 & 0.09 & 0.09 & 0.09 & 0.65 & 0.11 & 0.10 & 0.00 & 6 \\
14 & 0.25 & 0.04 & 0.28 & 0.10 & 0.15 & 0.04 & 0.10 & 0.00 & 2 \\
15 & 0.11 & & 1.08 & & 0.01 & & 1.15 & & 1 \\
\hline
\end{tabular}

Tabla 4.5: Huecos de tensión en parque eólico. Proceso K-means clustering: identificación de clusters y variabilidad de longitudes

\begin{tabular}{cccccccccc}
\hline Cluster & $\operatorname{avg}\left(V_{\min }\right)$ & $\sigma\left(V_{\min }\right)$ & $\operatorname{avg}(\Delta t)$ & $\sigma(\Delta t)$ & $\operatorname{avg}\left(\Delta t\left(V_{\min }\right)\right.$ & $\sigma\left(\Delta t\left(V_{\min }\right)\right.$ & $\operatorname{avg}\left(\Delta t_{0,8}\right)$ & $\sigma\left(\Delta t_{0,8}\right)$ & Número de huecos \\
\hline 1 & 0.87 & 0.02 & 0.07 & 0.02 & 0.07 & 0.02 & 0.00 & 0.00 & 48 \\
2 & 0.62 & 0.02 & 0.11 & 0.04 & 0.05 & 0.03 & 0.09 & 0.04 & 43 \\
3 & 0.83 & 0.02 & 0.11 & 0.04 & 0.11 & 0.04 & 0.00 & 0.00 & 37 \\
4 & 0.88 & 0.02 & 0.01 & 0.01 & 0.01 & 0.01 & 0.00 & 0.00 & 31 \\
5 & 0.71 & 0.02 & 0.11 & 0.04 & 0.08 & 0.04 & 0.09 & 0.04 & 26 \\
6 & 0.81 & 0.04 & 0.90 & 0.20 & 0.90 & 0.20 & 0.10 & 0.27 & 24 \\
7 & 0.77 & 0.02 & 0.09 & 0.04 & 0.06 & 0.03 & 0.06 & 0.04 & 22 \\
8 & 0.83 & 0.05 & 0.55 & 0.07 & 0.53 & 0.06 & 0.15 & 0.26 & 16 \\
9 & 0.77 & 0.04 & 0.78 & 0.09 & 0.12 & 0.10 & 0.13 & 0.10 & 15 \\
10 & 0.53 & 0.04 & 0.15 & 0.10 & 0.07 & 0.05 & 0.13 & 0.10 & 9 \\
11 & 0.53 & 0.08 & 0.74 & 0.10 & 0.62 & 0.08 & 0.74 & 0.10 & 7 \\
12 & 0.20 & 0.14 & 0.09 & 0.01 & 0.03 & 0.03 & 0.09 & 0.01 & 6 \\
13 & 0.58 & 0.09 & 0.71 & 0.09 & 0.09 & 0.09 & 0.70 & 0.09 & 6 \\
14 & 0.65 & 0.04 & 0.30 & 0.11 & 0.28 & 0.10 & 0.30 & 0.11 & 2 \\
15 & 0.79 & & 2.23 & & 1.08 & & 1.08 & & 1 \\
\hline
\end{tabular}

Tabla 4.6: Huecos de tensión en parque eólico. Proceso K-means clustering: identificación de clusters y parámetros principales de tensión y duración 


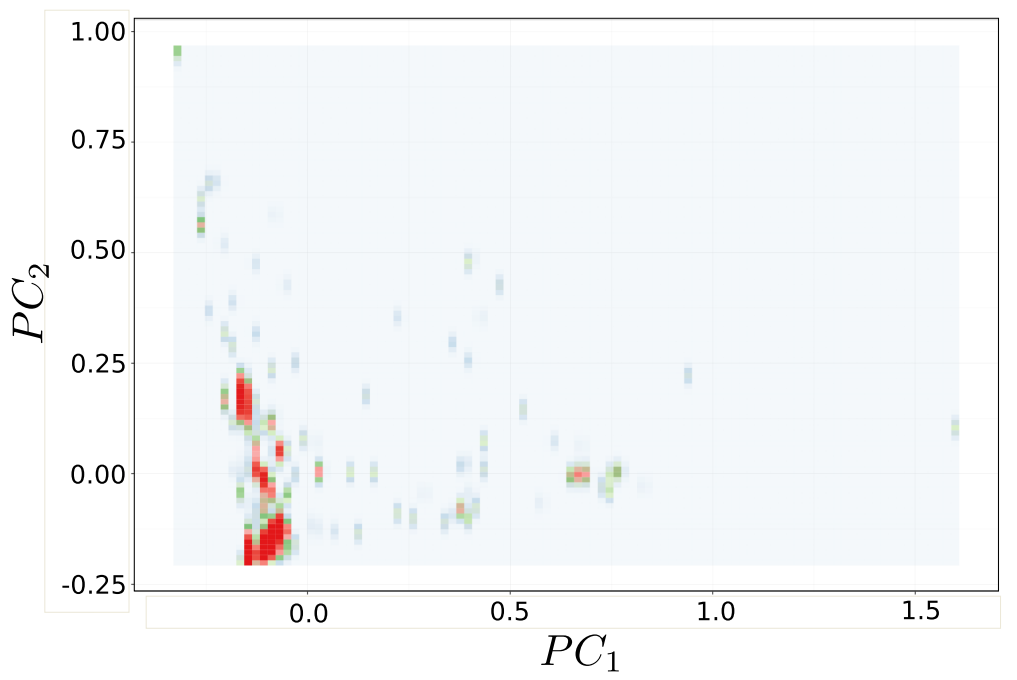

Figura 4.12: Huecos de tensión en parque eólico. Mapa de calor por caracterización de componentes $P C_{1}$ y $P C_{2}$.

Tal y como se comentó anteriormente, la identificación de estos grupos de eventos atendiendo a características similares permite proponer una serie de trayectorias promedio de tensiones eficaces aplicando técnicas de clustering basadas en $\mathrm{K}$-means. Estas trayectorias suponen patrones de evolución de tensiones eficaces a lo largo de la perturbación con las que comparar de manera ágil los requerimientos de operación, sin tener que representar todos los huecos medidos. Así, se estiman un número reducido de patrones los cuales proporcionan información notable sobre el comportamiento medio de cada cluster. Este análisis supone, pues, una vía alternativa para visualizar los eventos más esperados, deducidos de las campañas de medida en campo, evitando comparativas masivas e individuales que no son capaces de dar información general y global sobre la gran cantidad de eventos. De hecho, se ha detectado una laguna de contribuciones sobre este tema en la literatura específica. Por tanto, esta estimación de perfiles-tipo supone una destacable contribución de la presente tesis doctoral, aportando una nueva solución a un campo en el que las contribuciones han sido muy escasas. Como un ejemplo de la identificación del promedio de patrones, y con el objetivo de proporcionar una caracterización general y simplificada de las perturbaciones recogidas en comparación con los perfiles dados en los requerimientos de conexión español, la figura 4.13 muestra los diferentes patrones de tensión eficaz correspondientes a cada cluster para los huecos medidos en parque eólico. Estos perfiles-tipo se superponen con los límites de tensión eficaz permitidos por la normativa actual española, en un intento de visualizar no sólo la trayectoria promedio de los valores de tensión eficaz a lo largo de la perturbación, sino también su evolución en comparación con los límites 


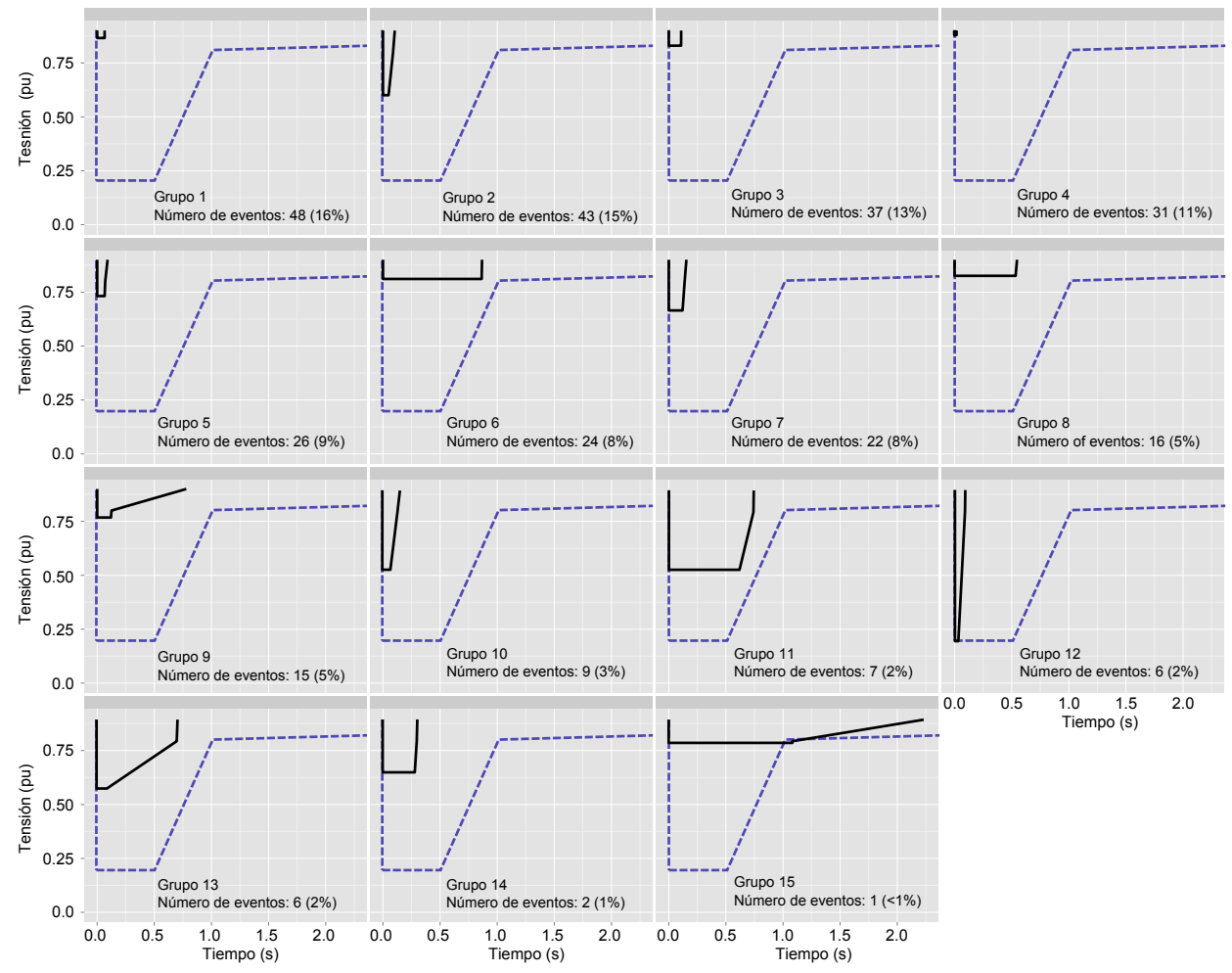

Figura 4.13: Huecos de tensión en parque eólico. Comparativa de patrones de tensiones eficaces con requerimientos de conexión español.

dados por las normativas actuales españolas. Como se puede ver, la mayoría de los eventos presentan claramente trayectorias de tensión eficaz que tienen que ser soportadas por las plantas de generación de energía renovable sin desconectarse de la red, y por lo tanto los protocolos españoles son relativamente severos en términos de máxima tensión eficaz residual sufridos por los sistemas de generación renovable. Al menos, atendiendo a los resultados derivados de estas campañas de medidas.

\subsubsection{Aplicación ACP y K-means a campañas de medida en instalaciones FV.}

Siguiendo un planteamiento similar al recogido en el apartado anterior (ver apartado 4.4.2, , se ha procedido de igual modo con los huecos medidos en instalaciones fotovoltaicas. El resultado del análisis por componentes principales se recoge en la tabla 4.7, donde puede comprobarse que la primera componente $P C_{1}$ engloba el $52 \%$ del total de la variabilidad, mientras que la segunda componente $P C_{2}$ añade casi el $39 \%$. En este caso, tanto la tercera componente $P_{3}$ como la cuarta $P C_{4}$ poseen una aportación muy reducida en ambos casos a la variabilidad total, lo que justificaría de forma 


\begin{tabular}{ccccc}
\hline & PC1 & PC2 & PC3 & PC4 \\
\hline Desviación estándar & 0,52 & 0,45 & 0,19 & 0,10 \\
Proporción de variabilidad & 0,52 & 0,39 & 0,07 & 0,02 \\
Proporción acumulada & 0,52 & 0,91 & 0,98 & 1 \\
\hline
\end{tabular}

Tabla 4.7: Huecos de tensión en instalación FV. Distribución de variabilidad por componentes principales.

\begin{tabular}{ccccc}
\hline & $l_{1}$ & $l_{2}$ & $l_{3}$ & $l_{4}$ \\
\hline$P C_{1}$ & $-0,28$ & 0,04 & $-0,96$ & $-0,08$ \\
$P C_{2}$ & $-0,01$ & 0,01 & 0,08 & -1.00 \\
$P C_{3}$ & $-0,10$ & $-0,99$ & $-0,01$ & $-0,01$ \\
\hline
\end{tabular}

Tabla 4.8: Huecos de tensíón en instalación FV. Coeficientes por componentes principales.

similar al caso anterior una reducción de cuatro parámetros $\left(l_{1}, l_{2}, l_{3}, l_{4}\right)$ a sólo dos parámetros $\left(P C_{1}, P C_{2}\right)$. Estos valores permiten que las cuatro longitudes iniciales en los que se ha dividido los huecos queden reducidas a sólo las dos primeras componentes principales del algoritmo ACP. Los coeficientes de las variables $l_{1}$ a $l_{4}$ en $P C_{1}, P C_{2}$ y $P C_{3}$ están recogidos en la tabla 4.8. La expresión asociada a las dos primeras componentes principales más significativas son, por tanto,

$$
\begin{aligned}
& P C_{1}, P V=0,28 l_{1}-0,04 l_{2}+0,96 l_{3}+0,08 l_{4} \\
& P C_{2}, P V=0,01 l_{1}-0,01 l_{2}-0,08 l_{3}+1 \cdot l_{4} .
\end{aligned}
$$

En este caso, la componente principal $P C_{1}$ está dominada mayoritariamente por $l_{3}$, mientras que para los huecos de tensión medidos en el parque eólico era $l_{2}$ la longitud de mayor aportación — ver tabla 4.4 —. Sin embargo, en este análisis de las campañas de medidas en instalaciones fotovoltaicas, la aportación de $l_{2}$ a $P C_{1}$ es prácticamente despreciable. La segunda componente básicamente depende de $l_{4}$, siendo el resto de valores muy bajos. Reduciendo las 4 longitudes a dos componentes principales se consigue más de un $90 \%$ de la variabilidad del grupo inicial de perturbaciones, un valor mayor que el conseguido al analizar mediante ACP el grupo de huecos medidos en el parque eólico.

La representación gráfica de estos resultados está recogida en la figura 4.14, donde cada uno de los 147 huecos medidos en las instalaciones FV es definido por sus parámetros según 4.8, y se encuentra etiquetado atendiendo al número de cluster al que pertenece. Estos huecos también se han representado mediante un mapa de color, figura 4.15, de esta forma se puede ver fácilmente la localización de los valores más habituales dentro del espacio bi-dimensional $P C_{1}$ y $P C_{2}$. La mayoría de los huecos están localizados en la 


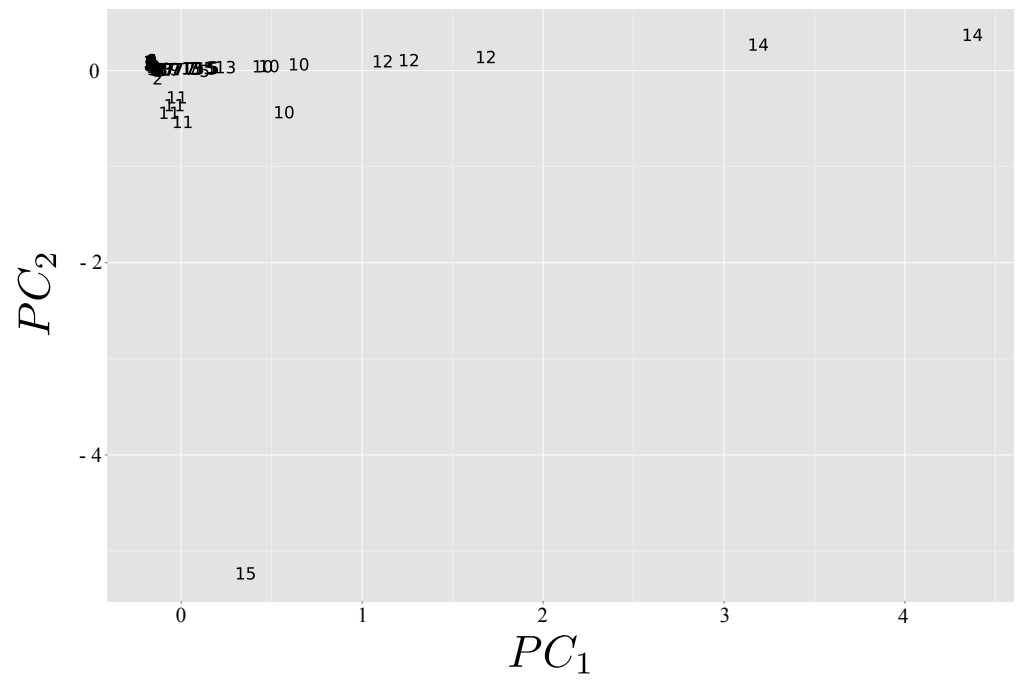

Figura 4.14: Huecos de tensión en instalaciones FV. Caracterización por componentes $P C_{1}$ y $P C_{2}$. Identificación de grupos y patrones mediante $\mathrm{K}$ means clustering.

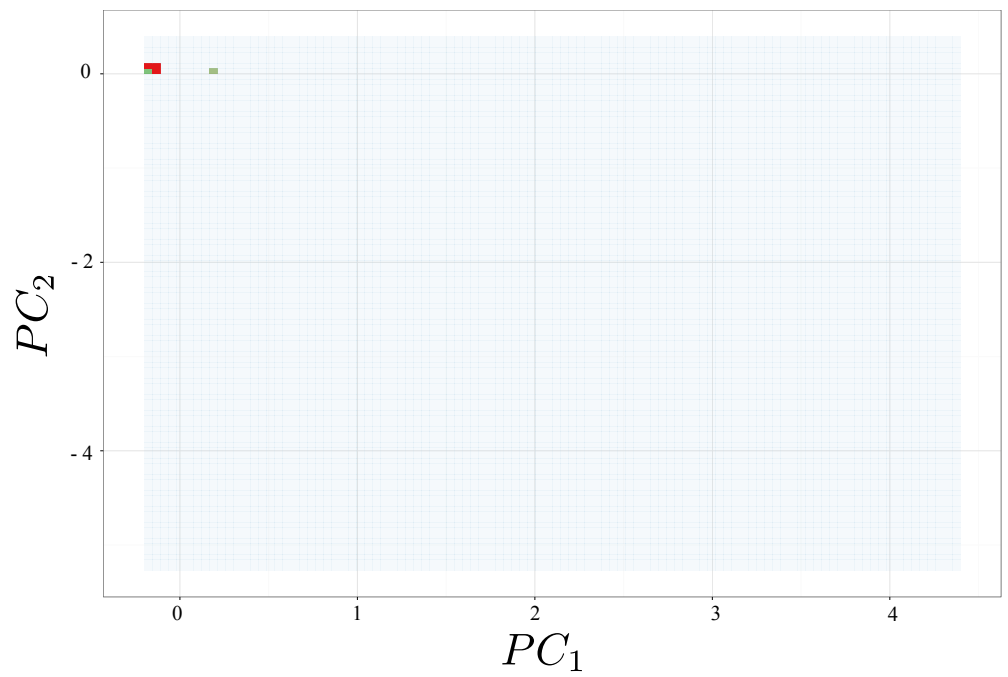

Figura 4.15: Huecos de tensión en instalaciones FV. Mapa de calor por caracterización de componentes $P C_{1}$ y $P C_{2}$. 
misma región. Son huecos con $P C_{2}$ prácticamente nulo, con lo cual quedan definidos por su componente $P C_{1}$, que al estar dominada por $l_{3}$ indican que la mayoría de los huecos son eventos en los que tiene una mayor relevancia la recuperación del hueco que el tiempo que éste pasa alrededor de su valor mínimo. Por tanto son huecos de poca permanencia alrededor del mínimo y en los que el período de recuperación de la tensión tiene, en general, mayor presencia que el tiempo alrededor del mínimo.

Atendiendo a la aplicación de las técnicas basadas en $\mathrm{K}$-means, y una vez definidos los diferentes clusters de perturbaciones y estimadas las trayectorias patrón de cada uno de los grupos, la figura 4.16 resume los perfiles-patrón de huecos analizados, comparando con los requerimientos actuales del sistema eléctrico español. Se observa claramente que son huecos en los que tiene mayor importancia la componente $l_{3}$ que $l_{2}$, y por tanto son eventos en los que la recuperación de los mismos posee mayor entidad que el tiempo alrededor del mínimo. Los grupos del 10 al 14 se van desplazando horizontalmente hacia valores más altos de $P C_{1}$, siendo huecos más profundos que el resto. Esto se confirma con la tabla 4.9 que muestra respectivamente la media y desviación estándar de cada longitud (desde $l_{1}$ a $l_{4}$ ) dentro de los 15 clusters. Finalmente, la tabla 4.10 resume los clusters, en términos de media y desviación típica, para los principales parámetros relacionados con los valores de tensión y duración.

\begin{tabular}{lrrrrrrrrr}
\hline cluster & mean(l1) & $\mathrm{sd}(11)$ & $\operatorname{mean}(12)$ & $\mathrm{sd}(12)$ & $\operatorname{mean}(13)$ & $\mathrm{sd}(13)$ & $\operatorname{mean}(14)$ & $\mathrm{sd}(14)$ & $\mathrm{n}$ \\
\hline 1 & 0.03 & 0.01 & 0.07 & 0.01 & 0.00 & 0.00 & 0.03 & 0.01 & 35 \\
2 & 0.08 & 0.02 & 0.08 & 0.03 & 0.00 & 0.01 & 0.09 & 0.03 & 21 \\
3 & 0.10 & 0.05 & 0.61 & 0.16 & 0.02 & 0.02 & 0.08 & 0.03 & 16 \\
4 & 0.02 & 0.01 & 0.02 & 0.01 & 0.00 & 0.00 & 0.02 & 0.01 & 14 \\
5 & 0.33 & 0.03 & 0.08 & 0.03 & 0.23 & 0.03 & 0.10 & 0.01 & 12 \\
6 & -0.00 & 0.01 & 0.00 & 0.00 & 0.00 & 0.00 & 0.00 & 0.00 & 11 \\
7 & 0.20 & 0.04 & 0.04 & 0.02 & 0.10 & 0.04 & 0.10 & 0.00 & 10 \\
8 & -0.03 & 0.01 & 0.00 & 0.00 & 0.00 & 0.00 & 0.03 & 0.01 & 6 \\
9 & 0.10 & 0.05 & 0.21 & 0.07 & 0.02 & 0.04 & 0.08 & 0.02 & 5 \\
10 & 0.50 & 0.09 & 0.03 & 0.02 & 0.57 & 0.11 & 0.22 & 0.25 & 4 \\
11 & 0.16 & 0.02 & 0.06 & 0.05 & 0.06 & 0.02 & 0.52 & 0.11 & 4 \\
12 & 0.75 & 0.13 & 0.00 & 0.00 & 1.36 & 0.28 & 0.10 & 0.00 & 3 \\
13 & 0.32 & 0.09 & 0.51 & 0.05 & 0.22 & 0.09 & 0.10 & 0.00 & 3 \\
14 & 0.89 & 0.00 & 0.02 & 0.02 & 3.86 & 0.88 & 0.10 & 0.00 & 2 \\
15 & 0.17 & & 0.05 & & 0.07 & & 5.36 & & 1 \\
\hline
\end{tabular}

Tabla 4.9: Huecos de tensión en instalaciones FV. Proceso K-means clustering: identificación de Clusters y variabilidad de longitudes. 

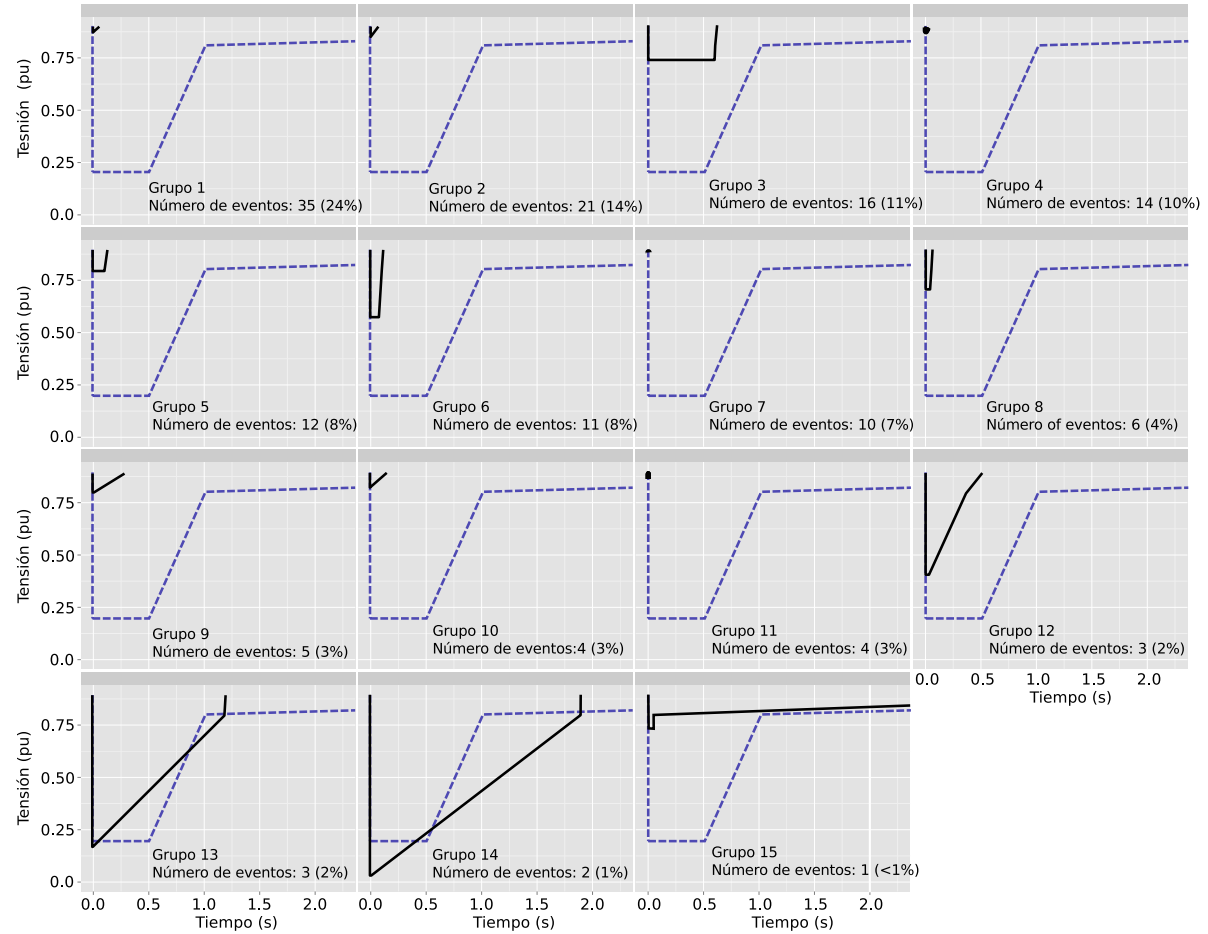

Figura 4.16: Huecos de tensión en instalaciones FV. Comparativa de patrones de tensiones eficaces con requerimientos de conexión español.

\begin{tabular}{|c|c|c|c|c|c|c|c|c|c|c|}
\hline cluster & mean(Vmin) & $\operatorname{sd}\left(V_{\min }\right)$ & mean(deltat) & sd(deltat) & mean(deltat_min) & sd(deltat_min) & mean(deltat_08) & sd(deltat_0 & 08) & $\overline{\mathrm{n}}$ \\
\hline 1 & 0.87 & 0.01 & 0.07 & 0.01 & 0.07 & 0.01 & 0.00 & & 0.00 & 35 \\
\hline 2 & 0.82 & 0.02 & 0.10 & 0.05 & 0.08 & 0.03 & 0.01 & & 0.02 & 21 \\
\hline 3 & 0.80 & 0.05 & 0.62 & 0.16 & 0.61 & 0.16 & 0.43 & & 0.34 & 16 \\
\hline 4 & 0.88 & 0.01 & 0.02 & 0.01 & 0.02 & 0.01 & 0.00 & & 0.00 & 14 \\
\hline 5 & 0.57 & 0.03 & 0.12 & 0.02 & 0.08 & 0.03 & 0.10 & & 0.02 & 12 \\
\hline 6 & 0.90 & 0.01 & 0.00 & 0.00 & 0.00 & 0.00 & 0.00 & & 0.00 & 11 \\
\hline 7 & 0.70 & 0.04 & 0.06 & 0.03 & 0.04 & 0.02 & 0.05 & & 0.03 & 10 \\
\hline 8 & 0.93 & 0.01 & 0.00 & 0.00 & 0.00 & 0.00 & 0.00 & & 0.00 & 6 \\
\hline 9 & 0.80 & 0.05 & 0.22 & 0.07 & 0.21 & 0.07 & 0.07 & & 0.10 & 5 \\
\hline 10 & 0.40 & 0.09 & 0.52 & 0.49 & 0.03 & 0.02 & 0.37 & & 0.28 & 4 \\
\hline 11 & 0.74 & 0.02 & 0.57 & 0.16 & 0.06 & 0.05 & 0.06 & & 0.06 & 4 \\
\hline 12 & 0.15 & 0.13 & 1.21 & 0.28 & 0.00 & 0.00 & 1.20 & & 0.28 & 3 \\
\hline 13 & 0.58 & 0.09 & 0.54 & 0.05 & 0.51 & 0.05 & 0.54 & & 0.05 & 3 \\
\hline 14 & 0.01 & 0.00 & 3.80 & 0.91 & 0.02 & 0.02 & 3.80 & & 0.91 & 2 \\
\hline 15 & 0.73 & & 5.41 & & 0.05 & & 0.05 & & & 1 \\
\hline
\end{tabular}

Tabla 4.10: Huecos de tensión en instalaciones FV. Proceso K-means clusteri$n g$ : identificación de Clusters y parámetros principales de tensión y duración. 



\section{Capítulo 5}

\section{Contributions and Future Work}

\section{ABSTRACT:}

In this last chapter, and according to the objectives previously established at the begining of the document, contributions are discussed in detail. Possible future work is included as well, as a brief summary of new proposals to be carried out by the candidate following the results and contributions already achieved in this thesis.

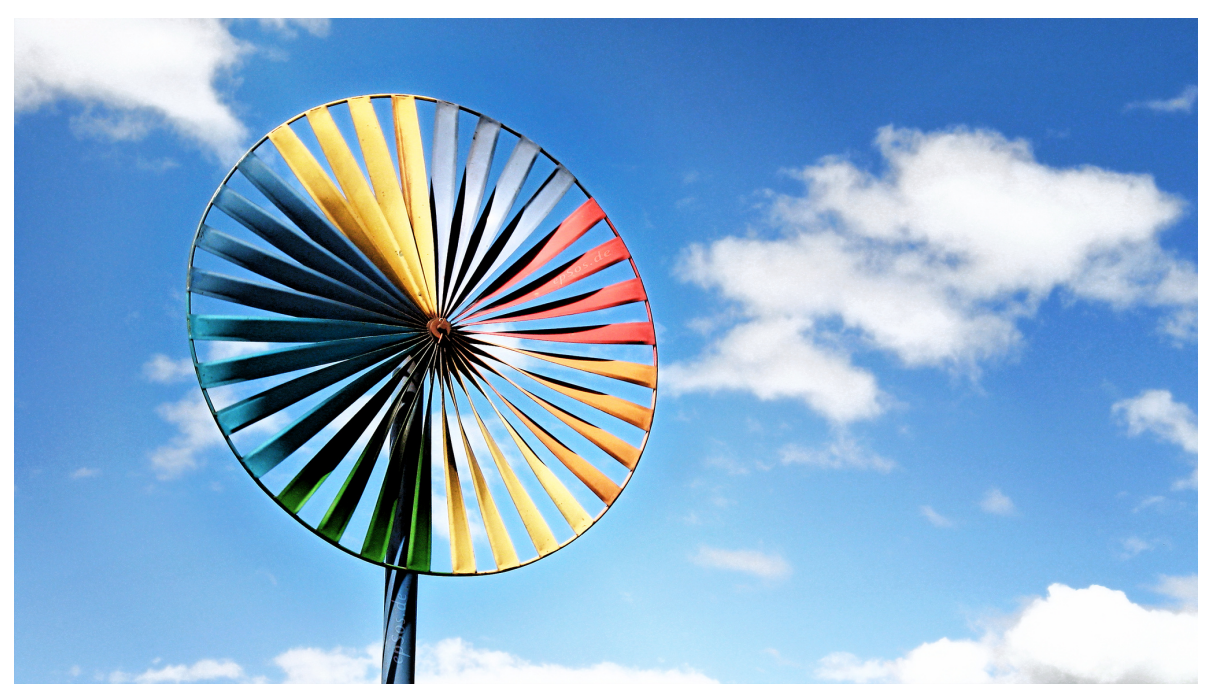

Wind Turbine for Renewable Electricity Generator (Source: http://epsos. de, Creative Commons Licence) 


\subsection{Contributions}

Considering the continuity requirements for renewable energy sources and the relevant insterest of some countries to promote the integration of these resources into power systems, specific grid-codes for the connection of generation units under disturbances have been developed and issued along the last years. Under this scenario, different objectives were discussed in Section 1.1. Based on these aims, conclusion and contributions are as follows:

- An additional parameter to characterize the severity of voltage dips is discussed and evaluated. This parameter accounts for the time interval around the minimum voltage value — residual rms voltage- . More specifically, the marginal time interval until the rms-voltage is recovering $10 \%$ higher than the residual rms-voltage. This additional parameter is suitable to characterize the severity of real collected events, allowing a proper comparison with current grid-code requirements as it was discussed in Section 3.4 .

- A set of four segment lenghts resulting from a linearization process of the real evolution along a voltage dip is proposed to identify rmsvoltage profiles under the presence of disturbances. This characterization has been applied on real voltage dips collected in Spanish wind farms and PV installations, linearizing the real rms-voltage profiles and providing intensive information in terms of the severity, duration and slopes during the fault without loss of relevant information. In comparison with previous approaches, this solution is an alternative and a more complete way to characterize disturbances and compare to norms and requirements. Actually, current grid-codes use linear profiles to define rms-voltage limits, and thus, the proposed linearization process offers a more convenient way to compare real events and norms currently in force.

- A process based on Principal Component Analysis and K-means clustering technique is proposed and implemented to deduce rms-voltage patterns as a reduced number of representative trajectories from a group of distrubances. The proposed methodology has been evaluated on a large amount of real events collected in Spanish wind farms and PV installations. For representation purposes, each voltage dip is characterized by the first two principal components $\left(P C_{1}\right.$ and $\left.P C_{2}\right)$, explaining most variability of the initial group of voltage dips and reducing the initial set of segment lengths from four to only two values. By applying K-means clustering, around 15 representative rms-voltage trajectories are estimated, reducing significantly the number of profiles to be compared to current requirements. This reduced group of 
rms-voltage profiles are then compared to the Spanish Grid-Code requirements, as a new attempt to assess the severity of current rules applied on generating units connected to the grid under the presence of disturbances. Moreover, these comparisons allow us to estimate the most likely rms-voltage dips, as well as their differences and similarities with grid requirements. This is a relevant contribution of this Thesis, since there is a lack of contributions addressing the characterization and clustering of a group of real voltage dips with the objective of comparing with current requirements, and estimating how severe and close to the rms-voltage limits the collected events are.

- An intensive assessment of the proposed methodology has been carried out by real disturbances collected in renewable installations connected to the Spanish distribution grid, which is also pointed out as a remarkable contribution of this Thesis. Actually, there are few contributions in the specific literature including a large amount of real data and disturbances.

These contributions have been the objetives of papers presented and published in different international conferences and journals. The aim of these papers directly linked with the present Thesis are outlined below.

- T. García-Sánchez, E.Gómez-Lázaro and A.Molina-García, "A Review and Discussion of the Grid-Code Requirements for Renewable Energy Sources in Spain", International Conference on Renewable Energies and Power Quality ICREPQ-14, Cordoba (Spain), 2014.

ABstRaCT: Both current and upcoming Spanish grid code requirements concerning renewable energy resources are discussed in this paper. Additionally, the last draft proposed by the Spanish transmission system operator is included as well, comparing the current specifications facing the new proposed requirements for renewable energy sources.

- T. García-Sánchez, E. Gómez-Lázaro, A. Gomes and A. Molina-García, "Integration of Renewable Energy Resources and Technical Requirements in European Distribution Networks", 4rd International Workshop on Integration of Solar Power into Power Systems, Berlin (Germany), 2014.

ABstract: Different Grid-Code requirements are described and discussed in terms of rms-voltage and time intervals, comparing criteria and specifications for the most integrated technologies: wind energy and PV installations. In addition, and in an attempt to provide general rules for new generator units connected to the grid, the last draft of Network Code on Requirements for Generators (developed by ENTSO-E) has been also included and discussed in the paper. 
- T. García-Sánchez, E.Gómez-Lázaro and A.Molina-García, "Integration of Renewable Energy Resources under Spanish Grid-Code Requirements: Study of Real Data Measurements in PV Power Plants", DYNA (doi:10.6036/7114), 2014, pp. 649-655.

ABSTRACT: This paper discusses the evolution of wind farms and PV power plants in the Spanish power system, describing the requirements and electrical behaviors of these sources under disturbances. Real field-test measurements carried out along several years in different Spanish PV power plants provide a realistic comparison between collected data and current grid-code requirements. This study, also included in the paper, offers a preliminary analysis regarding the severity of grid-code requirements in comparison with real disturbances.

- A. Honrubia-Escribano, T. García-Sánchez, E. Gómez-Lázaro, E. Muljadi and A.Molina-García, "Power Quality Surveys of Photovoltaic Power Plants: Characterization and Analysis of Grid-Code Requirements", IET Renewable Power Generation (doi:10.1049/iet-rpg.2014.0215), 2015, Accepted for publication.

ABSTRACT: The present paper analyses intensive power quality surveys carried out from 2008 to 2011 in three different Spanish PV power plants: a fixed array installation with $4 \mathrm{MW}$ PV power capacity, a PV power plant including dual axis-trackers with $1 \mathrm{MW}$ PV power capacity, and one more fixed array PV power plant with 5 MW PV power capacity. The time interval around the residual voltage is proposed and defined by the authors as an additional parameter to provide a complete characterization of the severity of the disturbances. In addition, this time interval also allows to compare in detail the collected data to the current European Grid-Code requirements.

\subsection{Future Work}

While this Thesis provides an alternative and practical approach to characterize voltage dips and compare with current requirements by means of estimated rms-voltage patterns, a sort of opportunities for extending the scope of this work remain. Some of these directions are following discussed:

- Regarding the application of clustering techniques to estimate rmsvoltage patterns, future efforts here could be targeted at refinement of this estimation by the use of alternatives methodologies. Particularly with the aim of optimizing the number of clusters to be identified.

- Currently, grid-code requirements are based on providing the rmsvoltage limits corresponding to the phase more affected by the event. The application of $d q$ transformation to propose grid-code requirements 


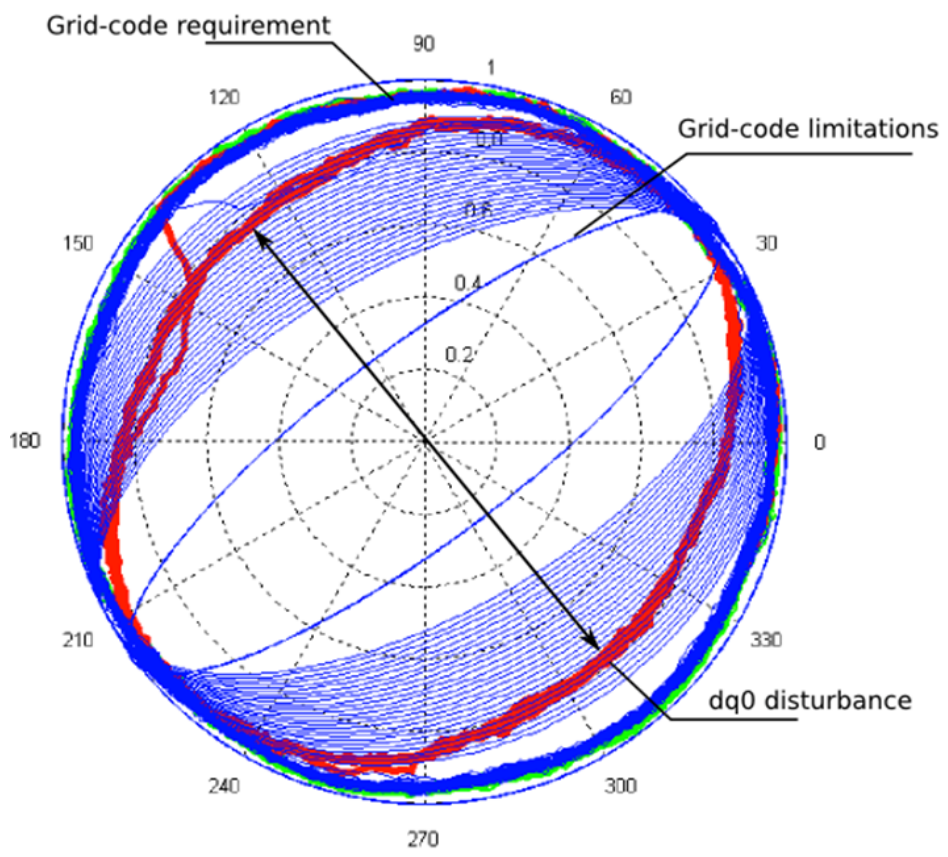

Figura 5.1: Proposal of Grid-Code Requirements by applying $d q$ transformation: comparison with a collected disturbance.

can certainly enhance those rules, considering not only the voltagephase more affected by the disturbance, but also the evolution of all phases in ordert to fix a global voltage and duration limits.

- In line with the previous item, the representation of voltage dips through $d q$ transformation is another potential way to represent the whole characteristics of the faults, as it was discussed in Section 3.2.2 A comparison between collected perturbances and requirements by means of $d q$ transformation is proposed as another future work. As an attempt to give preliminary results of this possible extension, figure 5.1 shows a comparison between estimated Spanish Grid-Code requirements by using $d q$ transformation and an example of a collected voltage dip. This representation gives a complete comparison of the trajectories followed by all phases, and depicts an intuitive visual representation based on the different shapes and forms provided by both rules and real events.

- By using $d q$ transformation, alternative techniques to deduce the corresponding rms-voltage patterns from a group of disturbances can be analyzed and proposed. Moreover, a systematic comparison between rules and measurements from the graphical representation of both $d q$ transformations needs to be addressed. In this way, it is proposed the 
estimation of geometric patterns and the comparison of shapes as an attempt to determine the severity of the faults and how close the gridcode requirements are.

- Finally, and under the current confidential agreement among Universidad Politécnica de Cartagena, Universidad de Castilla La Mancha and National Renewable Energy Laboratory (NREL, USA), it is also proposed as future work the application of Principal Component Analysis and K-means clustering on voltage dips collected in other power systems and voltage levels. This additional analysis can be useful as a comparison between different rms-voltage patterns, as well as an alternative way to estimate and visualize the severity of the events depending on the power systems considered. 


\section{Bibliografía}

[1] Constantinos Sourkounis and Pavlos Tourou. Grid code requirements for wind power integration in europe. In Conference Papers in Science, volume 2013. Hindawi Publishing Corporation, 2013.

[2] Math HJ Bollen and LD Zhang. Different methods for classification of three-phase unbalanced voltage dips due to faults. Electric power systems research, 66(1):59-69, 2003.

[3] Vanya Ignatova, Pierre Granjon, and Seddik Bacha. Space vector method for voltage dips and swells analysis. Power Delivery, IEEE Transactions on, 24(4):2054-2061, 2009.

[4] P.O.12.3 requisitos de respuesta frente a huecos de tension de las intalaciones de produccion de regimen especial. Red Electrica Española. Technical report, 2006.

[5] Erich W Gunther and H Mebta. A survey of distribution system power quality-preliminary results. Power Delivery, IEEE Transactions on, 10(1):322-329, 1995.

[6] Duarte Carvalho, João Wemans, João Lima, and Isabel Malico. Photovoltaic energy mini-generation: future perspectives for Portugal. Energy Policy, 39(9):5465-5473, 2011.

[7] Antonella Battaglini, Nadejda Komendantova, Patricia Brtnik, and Anthony Patt. Perception of barriers for expansion of electricity grids in the European Union. Energy Policy, 47:254-259, 2012.

[8] World Energy Outlook 2013. Technical report, International Egency Agency (IEA), 2012.

[9] IEA. Technology roadmap - solar photovoltaic energy. Technical report, International Energy Agency, 2010.

[10] D. Lew, L. Bird, E. Carlini, A. Estanqueiro, D. Flynn, E. GomezLazaro, B. Lange, N. Menemenlis, A. Orths, I. Pineda, C. Smith, L. Soder, P. Sorensen, Y. Yasuda, and H. Holttinen. Wind and solar 
curtailment: International experience and practices. In International Workshop on Large-Scale Integration of Wind Power into Power Systems as well as on Transmission Networks for Offshore Wind Farms, pages 1-7, London, United Kingdom, October 2013. Energynautics $\mathrm{GmbH}$.

[11] IEEE Recommended Practice for Monitoring Electric Power Quality, 2009.

[12] M.H.J. Bollen. Comparing voltage dips survey results. IEEE Power Engineering Society Winter Meeting, 2:1130 - 1134, 2002.

[13] T. A. Short. Electric Power Distribution Handbook. CRC Press, 2004.

[14] A. Moreno-Munoz and J. De la Rosa. Voltage sag in highly automated factories. IEEE Industry Applications Society Annual Meeting, pages $1-6,2008$.

[15] E. Gómez-Lázaro, J. A. Fuentes, A. Molina-García, and M. Canas Carreton. Characterization and visualization of voltage dips in wind power installations. IEEE Transactions on Power Delivery, 24, no. 4:2071 - 2078, 2009.

[16] Andrés Honrubia Escribano, Emilio Gómez-Lázaro, Graciano JiménezMoreno, and Angel Molina-García. Analysis of the AC-Contactor Electrical Behavior under Voltage Dips. XIX International Conference on Electrical Machines, 2010.

[17] E. Paal, Z. Weitzl, and C.S. Choi. Grid management functions built in $\mathrm{PV}$ inverters for distributed power generation. IEEE 8th International Conference on Power Electronics and ECCE, pages 2637-2644, 2011.

[18] A. Honrubia-Escribano, E. Gómez-Lázaro, A. Molina-García, and J.A. Fuentes. Influence of voltage dips on industrial equipment: Analysis and assessment. International Journal of Electrical Power 83 Energy Systems, 41, no. 1:87-95, 2012.

[19] Robert Passey, Ted Spooner, Iain MacGill, Muriel Watt, and Katerina Syngellakis. The potential impacts of grid-connected distributed generation and how to address them: A review of technical and nontechnical factors. Energy Policy, 39(10):6280-6290, 2011.

[20] Robert Passey, Ted Spooner, Iain MacGill, Muriel Watt, and Katerina Syngellakis. The potential impacts of grid-connected distributed generation and how to address them: A review of technical and nontechnical factors. Energy Policy, 39(10):6280-6290, 2011. 
[21] Alberto J. Lamadrid and Tim Mount. Ancillary services in systems with high penetrations of renewable energy sources, the case of ramping. Energy Economics, 34(6):1959 - 1971, 2012.

[22] C. Clastres, T.T. Ha Pham, F. Wurtz, and S. Bacha. Ancillary services and optimal household energy management with photovoltaic production. Energy, 35(1):55 - 64, 2010.

[23] Destenie Nock, Venkat Krishnan, and James D. McCalley. Dispatching intermittent wind resources for ancillary services via wind control and its impact on power system economics. Renewable Energy, 71(0):396 400, 2014.

[24] Natalia Sangroniz, José Arturo Mora, and Mateus Duarte Teixeira. Review of international grid codes for wind generation. 2009.

[25] Wind in power 2012 European statistics. Technical report, The European Wind Energy Association EWEA, February 2012.

[26] W. Christiansen and D. T. Johnsen. Analysis of requirements in selected grid codes. Technical report, Section of Electric power Engineering - Technical University of Denmark (DTU)., January 2006.

[27] Lidong Zhan and Math HJ Bollen. Characteristic of voltage dips (sags) in power systems. Power Delivery, IEEE Transactions on, 15(2):827832,2000 .

[28] Emilio Gómez-Lázaro, Juan Alvaro Fuentes, Angel Molina-García, and Miguel Cañas-Carretón. Characterization and visualization of voltage dips in wind power installations. Power Delivery, IEEE Transactions on, 24(4):2071-2078, 2009.

[29] M.I. Muhamad, N. Mariun, and Mohd Amran Mohd Radzi. The effects of power quality to the industries. 5th Student Conference on Research and Development, 2007.

[30] E. Muljadi, Z. Mills, R. Foster, J. Conto, and A Ellis. Fault analysis at a wind power plant for one year of observation. In Power and Energy Society General Meeting - Conversion and Delivery of Electrical Energy in the 21st Century, 2008 IEEE, pages 1-7, July 2008.

[31] M. Bollen, M. Stephens, S. Djokic, K. Stockman, B. Brumsickle, J. Milanovic, J Romero Gordón, R. Neumann, G. Ethier, F. Córcoles, A. Ferguson, P. Goossens, P. Ligot, A. Lopes Leiria, P. Marteyn, A. McEachern, J. Mentzer, I. McMichael, U. Minnaar, K. Reusel, and F. Zavoda. Voltage dip immunity of equipment and installations. Technical report, CIGRE, 2010. 
[32] S. Elphick, V. Smith, V. Gosbell, and R. Barr. The australian long term power quality survey project update. 14th International Conference on Harmonics and Quality of Power, pages 1-7, 2010.

[33] A. Honrubia-Escribano, E. Gómez-Lázaro, A. Molina-García, and J.A. Fuentes. Influence of voltage dips on industrial equipment: Analysis and assessment. International Journal of Electrical Power 63 Energy Systems, 41(1):87-95, 2012.

[34] M. Sturc. Energy consumption down by $8 \%$ between 2006 and 2012 in the eu28. Technical report, Eurostat Press Office, 2014.

[35] Carl E. Behrens. Energy policy: 113th congress issues. In Congressional Research Service, 2013.

[36] Fredrik Pettersson, Patrik Söderholm, and Robert Lundmark. Fuel switching and climate and energy policies in the european power generation sector: a generalized leontief model. Energy Economics, 34(4):1064-1073, 2012.

[37] Key world energy. Technical report, International Energy Agency, 2012.

[38] World Energy Outlook 2012. Technical report, International Energy Agency, 2012.

[39] C-E Stephan and Z Baba. Specifying a turbogenerator's electrical parameters guided by standards and grid codes. In Electric Machines and Drives Conference, 2001. IEMDC 2001. IEEE International, pages 63-68. IEEE, 2001.

[40] Wind in power. Technical report, European Wind Energy Association, February 2014.

[41] Monthly electricity statistics. Technical report, International Energy Agency, January 2014.

[42] Hannele Holttinen, Peter Meibom, Antje Orths, Bernhard Lange, Mark O'Malley, John Olav Tande, Ana Estanqueiro, Emilio Gomez, Lennart Söder, Goran Strbac, et al. Impacts of large amounts of wind power on design and operation of power systems, results of IEA collaboration. Wind Energy, 14(2):179-192, 2011.

[43] M. Milligan, D. Lew, H. Holttinen, J. Kiviluoma, I. Pineda, E. GómezLázaro, A. Estanqueiro, J. C. Smith, and S. Mueller. Wind integration cost and cost-causation: An iea task 25 collaboration. In International Workshop on Large-Scale Integration of Wind Power into Power Systems as well as on Transmission Networks for Offshore Wind Farms, 
pages 1-6, London, United Kingdom, October 2013. Energynautics $\mathrm{GmbH}$.

[44] H. Holttinen, A. Robitaille, A. Orths, B. Lange, M. O’Malley, A. Estanqueiro, M. Milligan, A. Rygg Aardaal, I. Pineda, E. Carlini, M. Gibescu, Y. Yasuda, E. Gomez, and L. Soder. Iea wind task 25 - summary of experiences and studies for wind integration. In International Workshop on Large-Scale Integration of Wind Power into Power Systems as well as on Transmission Networks for Offshore Wind Farms, pages 1-10, London, United Kingdom, October 2013. Energynautics GmbH.

[45] Y. Yasuda, A. R. Ordal, E. M. Carlini, A. Estanqueiro, D. Flynn, E. Gómez-Lázaro, D. H. Hernand, H. Holttinen, F. Van Hulle, J. Kiviluoma, J. Kondoh, B. Lange, N. Menemenlis, M. Milligan, A. Orths, C. Smith, and L. Soder. Flexibility chart. evaluation on diversity of flexibility in various areas. In International Workshop on Large-Scale Integration of Wind Power into Power Systems as well as on Transmission Networks for Offshore Wind Farms, London, United Kingdom, October 2013. Energynautics GmbH.

[46] A. Orths, H. Abildgaard, F. van Hulle, J. Kiviluoma, B. Lange, M. OMalley, D. Flynn, A. Keane, J. Dillon, E. M. Carlini, J. O. Tande, A. Estanqueiro, E. Gómez-Lázaro, L. Soder, M. Milligan, J. C. Smith, and C. Clark. Expert group report on recommended practices. 16 wind integration studies. International Energy Agency Implementing Agreement for Co-operation in the Research, Development, and Deployment of Wind Energy Systems, Helsinki, Finland, September 2013.

[47] S. Martín-Martínez, A. Vigueras-Rodríguez, E. Gómez-Lázaro, A. Molina-García, E. Muljadi, and M. Milligan. Advances in wind power, chapter Operation of power systems with large amounts of wind power: A view from the Spanish case, page 373. Intech, Rijeka, Croatia, November 2012.

[48] Hannele Holttinen, Juha Kiviluoma, Ana Estanqueiro, Tobias Aigner, Yih-Huei Wan, and Michael Milligan. Variability of load and net load in case of large scale distributed wind power. 2010.

[49] J. Zhang, B.-M. Hodge, J. Miettinen, H. Holttinen, E. Gómez-Lázaro, J. Dobschinski, R. Verzijbergh, and N. Cutululis. Analysis of variability and uncertainty in wind power forecasting: An international comparison. In International Workshop on Large-Scale Integration of Wind Power into Power Systems as well as on Transmission Networks for Offshore Wind Farms, pages 1-6, London, United Kingdom, October 2013. Energynautics GmbH. 
[50] H. Holttinen, J. Kiviluoma, A. Estanqueiro, E. Gómez-Lázaro, B. Rawn, J. Dobschinski, P. Meibom, E. Lannoye, T. Aigner, Y. H. Wan, and M. Milligan. Variability of load and net load in case of large scale distributed wind power. In International Workshop on LargeScale Integration of Wind Power into Power Systems as well as on Transmission Networks for Offshore Wind Farms, pages 1-6, Aarhus, Denmark, October 2011. Energynautics GmbH.

[51] Información básica de los sectores de la energía. Technical report, Comisión Nacional de la Energía, 2013.

[52] El sistema eléctrico español. Technical report, Red Eléctrica de España, (REE), 2013.

[53] Global Market Outlook for Photovoltaics Until 2016. Technical report, European Photovoltaic Industry Association, 2012.

[54] Wind in power 2013 European statistics. Technical report, The European Wind Energy Association EWEA, February 2013.

[55] Global Market Outlook for Photovoltaic 2014-2018. EPIA. Technical report, European Photovoltaic Industry Association EPIA, 2014.

[56] International energy agency. photovoltaic power systems programme. Technical report, International Energy Agency.

[57] Global Market Outlook for Photovoltaic 2013-2017. Technical report, European Photovoltaic Industry Association, 2013.

[58] Plan de Energías Renovables 2011-2020. Technical report, Noviembre 2001.

[59] P. Vethman L.W.M. Beurskens, M. Hekkenberg. Renewable energy projections as published in the national renewable energy action plans of the european member states. Technical report, European Environment Agency (EEA), November 2011.

[60] Youngsang Bae, T-K Vu, and R-Y Kim. Implemental control strategy for grid stabilization of grid-connected PV system based on german grid code in symmetrical low-to-medium voltage network. Energy Conversion, IEEE Transactions on, 28(3):619-631, 2013.

[61] Farid Katiraei, Reza Iravani, Nikos Hatziargyriou, and Aris Dimeas. Microgrids management. Power and Energy Magazine, IEEE, 6(3):54$65,2008$. 
[62] María Paz Comech, Miguel García-Gracia, Susana Martín Arroyo, and Miguel Ángel Martínez Guillén. Wind farms and grid codes. From Turbine to Wind Farms Technical Requirements and Spin-Off Products, page 17, 2011.

[63] I Erlich, W Winter, and A Dittrich. Advanced grid requirements for the integration of wind turbines into the german transmission system. In Power Engineering Society General Meeting. IEEE, 2006.

[64] Antonios Marinopoulos, Fabio Papandrea, Muhamad Reza, Staffan Norrga, Filippo Spertino, and Roberto Napoli. Grid integration aspects of large solar PV installations: LVRT capability and reactive powervoltage support requirements. In PowerTech, 2011 IEEE Trondheim, pages 1-8. IEEE, 2011.

[65] Jahangir Hossain and Apel Mahmud. Renewable energy integration. 2014.

[66] Mansour Mohseni and Syed M Islam. Review of international grid codes for wind power integration: Diversity, technology and a case for global standard. Renewable and Sustainable Energy Reviews, 16(6):3876$3890,2012$.

[67] István Erlich and Udo Bachmann. Grid code requirements concerning connection and operation of wind turbines in Germany. In Power Engineering Society General Meeting, pages 1253-1257. IEEE, 2005.

[68] BDEW Bundesverband der Energie- und Wasserwirtschaft e.V. Technical guideline generating plants connected to the medium-voltage network guideline for generating plants connected to and parallel operation with the medium-voltage network. Technical report, BDEW Bundesverband der Energie- und Wasserwirtschaft e.V., June 2008.

[69] E. Troester. New German grid codes for connecting PV systems to the medium voltage power grid. In 2nd International workshop on concentrating photovoltaic power plants: optical design, production, grid connection, pages 1-4, 2009.

[70] D Muñoz, A Kortajarena, and L Pedrosa. La generación distribuida: un paradigma emergente para el sector eléctrico. Dyna, 77(6):29-33, 2002 .

[71] Royal decree $436 / 2004$, on the methodology for updating and systematization of the legal and economic framework of the activity of electricity production in particular regimen is established. (In Spanish). Technical report, Ministerio de Economía, March 2004. 
[72] $\mathrm{BOE}^{\circ}$ 254. Resolution of October 4, 2006, the General Secretariat of Energy, by the method of Operation 12.3 Requirements approving response against voltage dips wind farms. (In Spanish). Technical report, Ministerio de Industria, Turismo y Comercio, October 2006.

[73] Royal Decree 1565/2010, by which regulates and modifies certain aspects of the activity of production of electric energy in special regime. (In Spanish). Technical report, November 2010.

[74] Ley 24/2013, de 26 de diciembre, del sector eléctrico, boe. Technical Report No. 310, Ministerio de Industria, Turismo y Comercio, December 2013 .

[75] Francisco Jiménez, Emilio Gómez-Lázaro, Juan Alvaro Fuentes, Angel Molina-García, and Antonio Vigueras-Rodríguez. Validation of a double fed induction generator wind turbine model and wind farm verification following the Spanish grid code. Wind Energy, 15(4):645-659, 2012.

[76] ENEL. Dk 5400 - criteria for the connection of customers to the network at distribution (In Italian). Technical report, ENEL, October 2004.

[77] ENEL. DK 5740 - Standards for connection to production facilities to the MV network of ENEL distribution (In Italian). Technical report, ENEL, February 2005.

[78] TERNA. Code of transmission, dispatching, development and network. (In Italian). Technical report, TERNA, 2006.

[79] F. Blaabjerg F. Lov. UNIFLEX-PM. advanced power converters for universal and flexible power management in future electricity network converter applications in future European electricity network. Technical report, Deliverable D2.1, EC Contract no. 019794(SES6), February 2007.

[80] Martin Braun, Thomas Stetz, Roland Bründlinger, Christoph Mayr, Kazuhiko Ogimoto, Hiroyuki Hatta, Hiromu Kobayashi, Ben Kroposki, Barry Mather, Michael Coddington, et al. Is the distribution grid ready to accept large-scale photovoltaic deployment? state of the art, progress, and future prospects. Progress in Photovoltaics: Research and Applications, 20(6):681-697, 2012.

[81] Separata del Borrador de P.O.12.2 Requisitos Técnicos de las intalaciones eólicas, fotovoltaicas y todas aquellas instalaciones de producción cuya tecnología no emplee un generador síncrono conectado directamente a la red. 
[82] D.F. Montoro. Recommendations for unified technical regulations for grid-connected PV systems. Technical report, SUNRISE project - European Photovoltaic Industry Association, the European Construction Industry Federation, the European Association of Electrical Contractors, International Union of Architects, 2009.

[83] B.I. Craciun, T. Kerekes, D. Sera, and R. Teodorescu. Overview of recent grid codes for PV power integration. In 13th International Conference on Optimization of Electrical and Electronic Equipment (OPTIM), pages 959-965, May 2012.

[84] Network code requirements for grid connection applicable to all generators. Technical report, European Network of Transmission System Operators for Electricity (ENTSO-E), October 2013.

[85] Maria van der Hoeven. How we must build the electric future. Energy IEA, 6:3, 2014.

[86] J Balcells. Calidad de la red eléctrica: cómo medirla. Online: http://www. jcee. upc. es/JCEE2001/PDFs, 202000, 2001.

[87] 1159-2009-IEEE Recommended practice for monitoring electric power quality. Technical report, June 2009.

[88] Daniele Gallo, Carmine Landi, and Mario Luiso. Accuracy analysis of algorithms adopted in voltage dip measurements. Instrumentation and Measurement, IEEE Transactions on, 59(10):2652-2659, 2010.

[89] Thomas Allen Short. Electric power distribution handbook. CRC press, 2014.

[90] D Daniel Sabin, Thomas E Grebe, and A Sundaram. RMS voltage variation statistical analysis for a survey of distribution system power quality performance. In Power Engineering Society 1999 Winter Meeting, IEEE, volume 2, pages 1235-1240. IEEE, 1999.

[91] KJ Cornick and HQ Li. Power quality and voltage dips: problem, requirements, responsibilities. 2000.

[92] M. Bollen. Understanding Power Quality Problems (Voltage Sags and Interruptions). New York: Wiley-IEEE Press, 2000.

[93] JA Parle, M Madrigal, and E Acha. Trends in power quality monitoring. Power Engineering Review, 21(10):3-21, 2001.

[94] Dong-Jun Won, Seon-Ju Ahn, and Seung-Il Moon. A modified sag characterization using voltage tolerance curve for power quality diagnosis. Power Delivery, IEEE Transactions on, 20(4):2638-2643, 2005. 
[95] Ali Chowdhury and Don Koval. Power distribution system reliability: practical methods and applications, volume 48. John Wiley \& Sons, 2011.

[96] Douglas S Dorr, M Brent Hughes, Thomas M Gruzs, Robert E Jurewicz, and John L. McClaine. Interpreting recent power quality surveys to define the electrical environment. Industry Applications, IEEE Transactions on, 33(6):1480-1487, 1997.

[97] Don O Koval, Romela A Bocancea, Kai Yao, and M Brent Hughes. Canadian national power quality survey: frequency and duration of voltage sags and surges at industrial sites. Industry Applications, IEEE Transactions on, 34(5):904-910, 1998.

[98] Paulo Gomes, ACB Martins, CR Zani, and SLA Sardinha. Connection requirements and grid codes for distributed generation. In Integration of Wide-Scale Renewable Resources Into the Power Delivery System, 2009 CIGRE-IEEE PES Joint Symposium, pages 1-12. IEEE, 2009.

[99] Iec 61000-4-30: Testing and measurement techniques. power quality measurement methods. Technical report, 2003.

[100] Math HJ Bollen. Algorithms for characterizing measured three-phase unbalanced voltage dips. Power Delivery, IEEE Transactions on, 18(3):937-944, 2003.

[101] Roberto C Leborgne and Daniel Karlsson. Phasor based voltage sag monitoring and characterisation. In Electricity Distribution, 2005. CIRED 2005. 18th International Conference and Exhibition on, pages 1-4. IET, 2005.

[102] Sasa Z Djokic, Jovica V Milanovic, David J Chapman, Mark F McGranaghan, and Daniel S. Kirschen. A new method for classification and presentation of voltage reduction events. Power Delivery, IEEE Transactions on, 20(4):2576-2584, 2005.

[103] Chris Fitzer, Mike Barnes, and Peter Green. Voltage sag detection technique for a dynamic voltage restorer. In Industry Applications Conference, 2002. 37th IAS Annual Meeting Conference Record of the, volume 2, pages 917-924. IEEE, 2002.

[104] IEC/TR 61000-2-8 Electromagnetic compatibility (EMC) - Part 2-8: Environment - Voltage dips and short interruptions on public electric power supply systems with statistical measurement results. Technical report, 2002. 
[105] N Kagan, EL Ferrari, NM Matsuo, SX Duarte, A Sanommiya, JL Cavaretti, UF Castellano, and A Tenorio. Influence of rms variation measurement protocols on electrical system performance indices for voltage sags and swells. In Harmonics and Quality of Power, 2000. Proceedings. Ninth International Conference on, volume 3, pages 790-795. IEEE, 2000.

[106] Daniel L Brooks, Roger C Dugan, Marek Waclawiak, and Ashok Sundaram. Indices for assessing utility distribution system RMS variation performance. Power Delivery, IEEE Transactions on, 13(1):254-259, 1998.

[107] Math HJ Bollen. The influence of motor reacceleration on voltage sags. Industry Applications, IEEE Transactions on, 31(4):667-674, 1995.

[108] MHJ Bollen and PME Dirix. Simple model for post-fault motor behaviour for reliability-power quality assessment of industrial power systems. IEE Proceedings-Generation, Transmission and Distribution, 143(1):56-60, 1996.

[109] Math HJ Bollen. Characterisation of voltage sags experienced by threephase adjustable-speed drives. Power Delivery, IEEE Transactions on, 12(4):1666-1671, 1997.

[110] M. Pietzsch. Convertidores CC/CA para la conexión directa a red de sistemas fotovoltaicos: comparación entre topologías de 2 y 3 niveles. Bachelor thesis, UPC, 2004.

[111] Pedro Rodriguez, Remus Teodorescu, I Candela, Adrian Vasile Timbus, $\mathrm{M}$ Liserre, and F Blaabjerg. New positive-sequence voltage detector for grid synchronization of power converters under faulty grid conditions. In Power Electronics Specialists Conference, 2006. PESC'06. 37th IEEE, pages 1-7. IEEE, 2006.

[112] C Schauder and H Mehta. Vector analysis and control of advanced static VAR compensators. In IEE Proceedings C (Generation, Transmission and Distribution), volume 140, pages 299-306. IET, 1993.

[113] Robert H Park. Two-reaction theory of synchronous machines generalized method of analysis-part i. American Institute of Electrical Engineers, Transactions of the, 48(3):716-727, 1929.

[114] A Honrubia-Escribano, E Gómez-Lázaro, A Molina-Garcia, and S Martín-Martínez. Load influence on the response of ac-contactors under power quality disturbances. International Journal of Electrical Power E3 Energy Systems, 63:846-854, 2014. 
[115] A. Honrubia-Escribano, T. García-Sánchez, E. Gómez-Lázaro, E. Muljadi, and A. Molina-García. Power quality surveys of photovoltaic power plants: characterisation and analysis of grid-code requirements. IET Renewable Power Generation, 2014.

[116] Lorry E Conrad and Math HJ Bollen. Voltage sag coordination for reliable plant operation. Industry Applications, IEEE Transactions on, 33(6):1459-1464, 1997.

[117] Math HJ Bollen. Comparing voltage dip survey results. In Power Engineering Society Winter Meeting, 2002. IEEE, volume 2, pages 11301134. IEEE, 2002.

[118] A. Moreno-Muñoz and J. de la Rosa. Voltage sag in highly automated factories. In Industry Applications Society Annual Meeting. IAS2008., pages 1-6. IEEE, 2008.

[119] A. Honrubia-Escribano, A. Molina-García, E. Gómez-Lázaro, and E. Muljadi. Power quality survey of a photovoltaic power plant. In 39th IEEE Photovoltaic Specialists Conference, PVSC, Tampa, USA, 16-21 June 2013.

[120] K.J. Cornick and H.Q. Li. Power quality and voltage dips: problem, requirements, responsibilities. International Conference on Advances in Power System Control, Operation and Management., 1:149 - 156, 2000.

[121] H.N. Villegas Pico and D.C. Aliprantis. Voltage ride-through capability verification of wind turbines with fully-rated converters using reachability analysis. Energy Conversion, IEEE Transactions on, 29(2):392405, June 2014.

[122] T. Hastie, R. Tibshirani, and J. Friedman. The elements of statistical learning: data mining, inference and prediction. Springer, 2 edition, 2009. 